\title{
THOUGHT-ACTION FUSION AND INFLATED RESPONSIBILITY IN
} PSYCHOPATHOLOGY

\author{
By \\ Kirsty Jane Fraser
}

\author{
A thesis \\ submitted to Victoria University of Wellington \\ in fulfilment of the requirements for the degree of \\ Doctor of Philosophy \\ in Psychology
}

Victoria University of Wellington

July 2013 


\begin{abstract}
The aim of the current research was to investigate the presence and roles of inflated responsibility and thought-action fusion in psychopathology. The three underlying research themes were to examine the relationship between thought-action fusion and inflated responsibility, the roles that they play in psychopathology, and the possible etiology of these types of beliefs. It is proposed that these responsibility beliefs are not specific to obsessive compulsive disorder, as commonly assumed, and that they play important roles in the maintenance of a range of psychological symptoms. This thesis presents the results of four studies. The first study was designed to investigate the presence of Thought-Action Fusion (TAF) and Inflated Responsibility (IR) alongside symptoms of depression, anxiety, and obsessive-compulsive disorder, with thought suppression playing an intermediary role. Study 2 examined the interaction between responsibility beliefs and locus of control on obsessive-compulsive symptoms with non-clinical and clinical participants. The third study focussed on the etiology of responsibility beliefs, taking Salkovskis, Shafran, Rachman, and Freeston's (1999) theory of Pathways to Inflated Responsibility and empirically testing this with both clinical and non-clinical samples. Study 4 focussed on the relationship between responsibility beliefs and religiosity, using participants of Protestant Christian beliefs and Atheists. These studies collectively show that Thought-Action Fusion and Inflated Responsibility are both important and contributing factors in psychopathology, especially playing a part in the maintenance of symptoms and feelings of distress. Results indicated that while TAF tends to be specific to obsessive compulsive symptoms, IR is more of a general cognitive bias. Results also indicate that critical experiences in one's life can lead to biases in responsibility beliefs. Additionally, results show that these biases are not always indicative of psychopathology when they are acceptable within a particular set of morals, for example religion. These findings are of both theoretical and clinical significance because they add to the growing understanding of TAF and IR in psychopathology. The current research was conducted with observational, self-report measures; further research using longitudinal studies is needed for more clarity on causality.
\end{abstract}




\section{Acknowledgements}

Firstly I would like to thank my amazing primary supervisor, Associate Professor John McDowall for all the encouragement, support and guidance over the last few years. To my secondary supervisor, Associate Professor Matt Crawford, you are truly a writing genius, and I could not have got through the last few months without your feedback. You are a superstar; thank you for being on my side.

I would like to thank the staff at Anxiety Support Canterbury, particularly Nicky Weston and Ian Johnson for their voluntary support with this research through advertising my research to prospective participants. A big thank you also to the clients of this organisation who participated by completing my surveys.

A big thank you to the awesome folk who helped me out with my theological quandaries, and attempted to answer my ridiculous questions about religion and biblical text: Pastor Clint Craig, Associate Pastor Richard Ralston, Drew Harlow (Elder), Pastor Robyn Mellar-Smith and Dr. Andre Muller.

To my amazing friends, thank you all for being there, taking an interest, distracting me, providing support and offers of toddler watching, especially my sisters Linda and Julie, the beautiful Ralston family (I still can't believe you had family discussions about my research (:), Evi and Dion, Darshi, Mike, Paul, Josie, Crysta, Bekah Ralston (my stress buddy), Lisa Harlow and Mindy Craig. A super big thank you for the support I have received from LifeSwitch. And to Nigel Latta the rockstar for a well needed pep talk and boost of motivation in the final few weeks of writing.

To my beautiful family, thank you so much for everything. My mum and dad - you guys are the bestest, and this would not have been possible without your amazing help: proof-reading, reference checking, toddler watching, feeding me, listening; the list goes on and on. I love you guys to the moon and back. Thank you to my fabulous in-laws, Kerry and Yolanda for dinners, cake and helping with Lucas.

To my Dave. I would not have been able to do any of this without your support and love. You completely rock my world. And to my baby boy Lucas, thanks for sharing mummy over the last couple of years, and for making me laugh every single day. Love you boys to bits.

This thesis was completed with the assistance of the PhD Submission Scholarship. 
Table of Contents

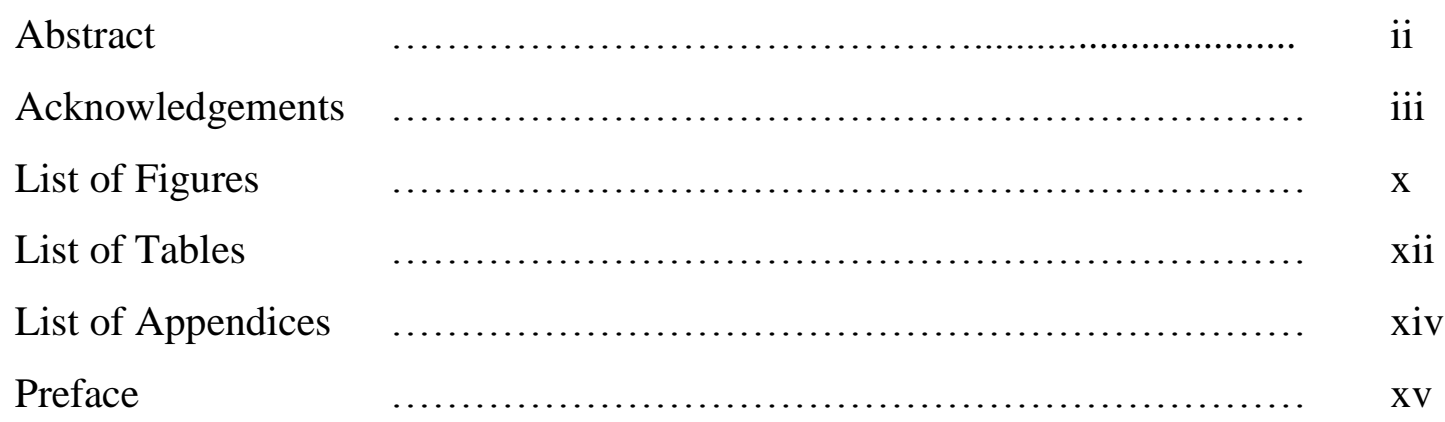

1. CHAPTER ONE: GENERAL INTRODUCTION AND LITERATURE REVIEW $\quad$............................................................. 1

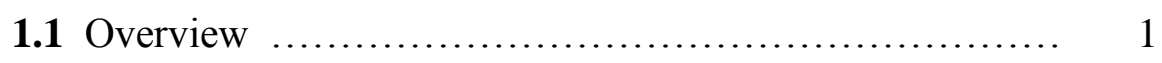

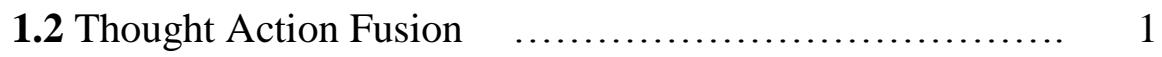

1.3 Inflated Responsibility $\quad$.............................. 3

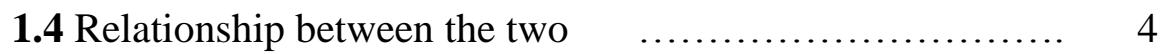

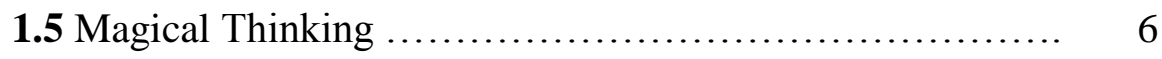

1.6 Obsessive Compulsive Disorder (OCD) ................ 7

1.6.1 OCD and TAF .............................. 7

1.6.2 OCD and Inflated Responsibility ............... 8

1.7 Cognitive Models ..................................... 8

1.7.1 Salkovskis' Cognitive Model of OCD ........... 8

1.7.1.1 Neutralising ........................... 10

1.7.2 Misinterpretation of Significance Theory ........ 11

1.8 TAF and Inflated Responsibility in other psychopathology. 13

1.8.1 Other Anxiety Disorders ...................... 13

1.8.2 Depression ..................................... 14

1.8.3 Eating Disorders .............................. 14

1.8.4 Psychotic Disorders .......................... 15

1.9 Religiosity ......................................... 16

1.10 Thought Suppression .............................. 17

1.11 Chapter Summary ................................. 18

1.12 The Current Research .................................. 18 
2. CHAPTER TWO: INFLATED RESPONSIBILITY AND THOUGHTACTION FUSION IN PSYCHOPATHOLOGY, AND THE ROLE OF THOUGHT SUPPRESSION ........................................ 20

2.1 Introduction .......................................... 20

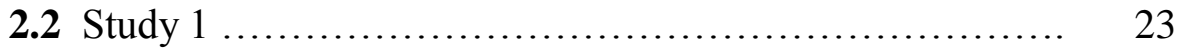

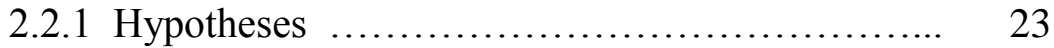

2.3 Method ........................................... 24

2.3.1 Participants .................................. 24

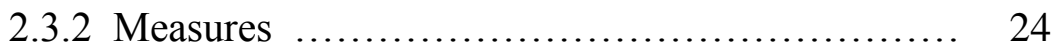

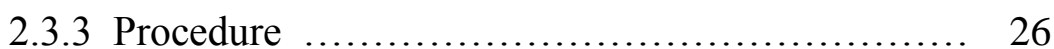

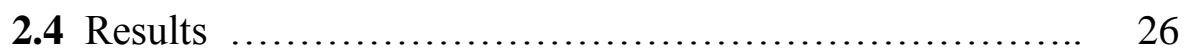

2.4.1 Descriptive Analyses $\ldots . \ldots \ldots \ldots \ldots \ldots \ldots \ldots \ldots .26$

2.4.2 Model Analyses ............................... 29

2.4.3 Measurement Model ........................... 29

2.4.4 Structural Modelling ............................ 31

2.4.4.1 Inflated Responsibility $\ldots \ldots \ldots \ldots \ldots \ldots . . \ldots 31$

2.4.4.2 Thought-Action Fusion $\ldots \ldots \ldots \ldots \ldots \ldots . . . . . .33$

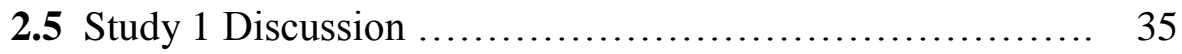

2.5.1 Clinical Implications $\ldots \ldots \ldots \ldots \ldots \ldots \ldots \ldots \ldots \ldots . . \ldots \ldots$

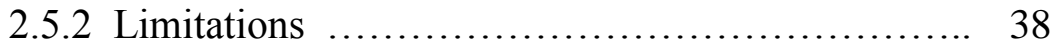

2.6 Chapter Summary ..................................... 39

3. CHAPTER THREE: RESPONSIBILITY BELIEFS AND PERCEIVED

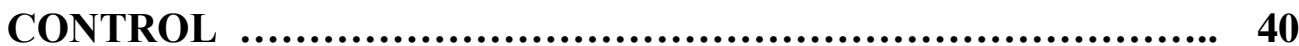

3.1 Control Beliefs and Obsessive Compulsive Disorder ....... 40

3.2 Locus of Control ...................................... 41

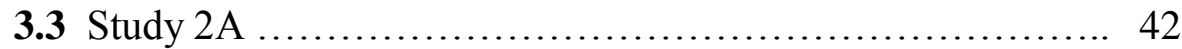

3.3.1 Hypotheses ................................. 42

3.4 Method ................................................ 43

3.4.1 Participants .................................. 43

3.4.2 Measures ....................................... 43

3.4.3 Procedure ..................................... 43

3.5 Results ............................................ 44

3.5.1 Descriptive Statistics $\ldots . \ldots \ldots \ldots \ldots \ldots \ldots \ldots \ldots \ldots . \ldots \ldots$ 
3.5.2 Multiple Regression $\ldots \ldots \ldots \ldots \ldots \ldots \ldots \ldots \ldots \ldots . \ldots 4$

3.5.3 Moderation Analyses ......................... 45

3.6 Study 2A Discussion ................................ 46

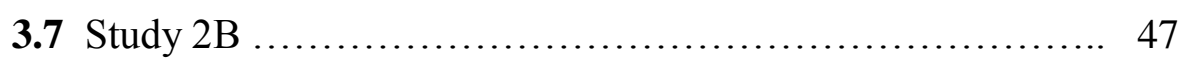

3.7.1 Hypotheses ............................... 47

3.8 Method ............................................ 47

3.8.1 Participants ................................. 47

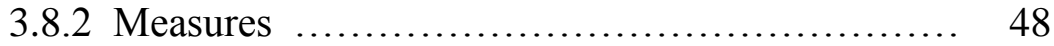

3.8.3 Procedure ..................................... 48

3.9 Results ............................................. 48

3.9.1 Descriptive Statistics ....................... 48

3.9.2 Multiple Regression .......................... 49

3.9.2.1 Inflated Responsibility ................. 49

3.9.2.2 Thought-Action Fusion ................ 50

3.9.2.2.1 TAF-likelihood and TAF-moral . 52

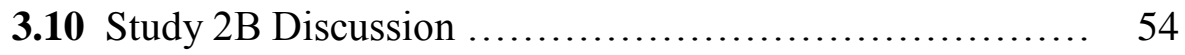

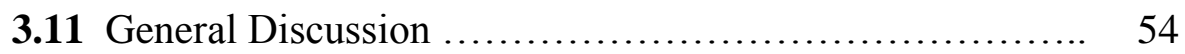

3.11.1 Clinical Implications ....................... 55

3.11.2 Limitations and Future Research .............. 55

3.12 Chapter Summary .................................. 56

\section{CHAPTER FOUR: PATHWAYS TO INFLATED RESPONSIBILITY} BELIEFS ...................................................... 57

4.1 Pathways to Inflated Responsibility .................... 57

4.1.1 Heightened Sense of Responsibility ............ 58

4.1.2 Rigid and Extreme Codes ..................... 59

4.1.3 Overprotection ................................ 59

4.1.4 Actions contributing to incident ................ 59

4.1.5 Perception of actions contributing to incident ...... 59

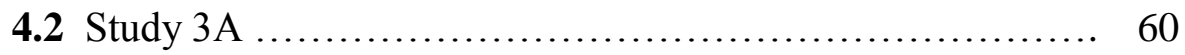

4.2.1 Hypotheses .................................. 61

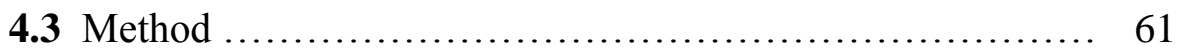

4.3.1 Participants .................................. 61

4.3.2 Measures ...................................... 62 
4.3.3 Procedure ..................................... 62

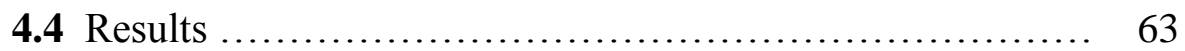

4.4.1 Descriptive Statistics ............................. 63

4.4.2 Regression Analyses ............................. 64

4.4.3 Mediation Analyses .............................. 65

4.5 Study 3A Discussion ..................................... 65

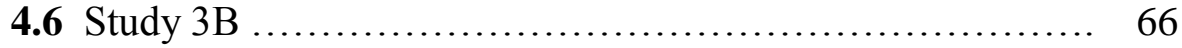

4.6.1 Hypotheses ...................................... 67

4.7 Method ..................................................... 67

4.7.1 Participants ........................................ 67

4.7.2 Measures ........................................ 67

4.7.3 Procedure ...................................... 68

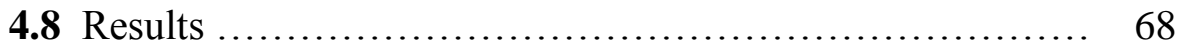

4.8.1 Descriptive Statistics ............................. 68

4.8.2 Inflated Responsibility ........................... 69

4.8.2.1 Regression Analyses .................... 69

4.8.2.2 Mediation Analyses .................... 70

4.8.3 Thought-Action Fusion ............................. 70

4.8.3.1 Regression Analyses .................... 70

4.8.3.2 Mediation Analyses .................... 71

4.8.4 Thought-Action Fusion- Likelihood …........... 72

4.8.4.1 Regression Analyses ..................... 72

4.8.4.2 Mediation Analyses ..................... 72

4.8.5 Thought-Action Fusion - Moral ................... 73

4.8.5.1 Regression Analyses .................... 73

4.8.5.2 Mediation Analyses ...................... 73

4.9 Study 3B Discussion ................................... 74

4.10 General Discussion .................................... 74

4.10.1 Clinical Implications …......................... 76

4.10.2 Limitations ......................................... 77

4.10.3 Future Research ................................. 77

4.11 Chapter Summary …..................................... 77 
5. CHAPTER FIVE: RELIGIOUS AFFILIATION AND

RESPONSIBILITY BELIEFS

5.1 Literature Review ...................................... 79

5.1.2 Religious Affiliation vs. Religiosity ............ 81

5.2 Study 4 ........................................... 81

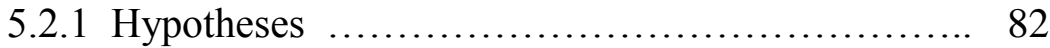

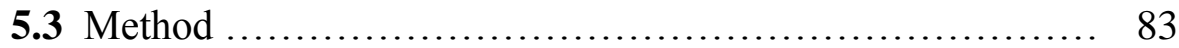

5.3.1 Participants ................................... 83

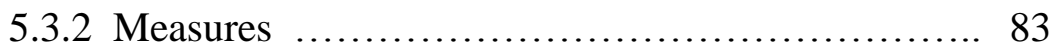

5.3.3 Procedure ....................................... 83

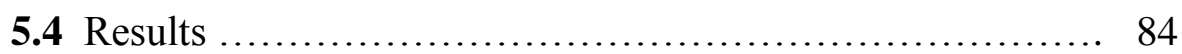

5.4.1 Descriptive Analyses ........................ 84

5.4.2 Group Differences ............................ 85

5.4.3 Moderation ....................................... 86

5.4.3.1 OC Symptoms ......................... 86

5.4.3.2 Depression ............................ 87

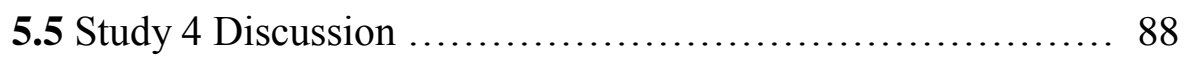

5.5.1 Christian Theology $\ldots . \ldots \ldots \ldots \ldots \ldots \ldots \ldots \ldots \ldots . \ldots 9$

5.5.2 Religious Affiliation ......................... 90

5.5.3 Clinical Implications .......................... 91

5.5.4 Limitations .................................... 91

5.5.5 Future Research ............................... 91

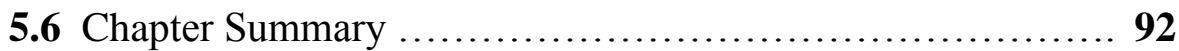

\section{CHAPTER SIX: RESEARCH SUMMARY AND GENERAL}

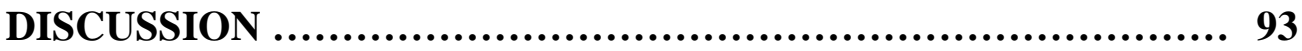

6.1 Research Summary ....................................... 93

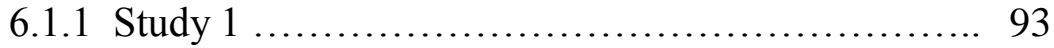

6.1.2 Studies 2A and 2B .......................... 94

6.1.3 Studies 3A and 3B ........................... 94

6.1 .4 Study 4 .................................. 95

6.2 The relationship between thought-action fusion and inflated responsibility .......................................... 95

6.2.1 Misinterpretation of Significance Theory ......... 96 
6.2.2 TAF-likelihood and TAF-moral ....

96

6.3 The roles of thought-action fusion and inflated responsibility in psychopathology 97

6.4 The etiology of thought-action fusion and inflated responsibility 99

6.5 Clinical Implications 99

6.6 Limitations and Directions for Future Research 101

6.7 Conclusions 102

7. REFERENCES 103

8. APPENDICES

113 


\section{List of Figures}

Figure 1.1 Inflated Responsibility (IR) and Thought-Action Fusion (TAF) ... 4

Figure 1.2 Inflated Responsibility (IR), Thought-Action Fusion (TAF), and Magical Thinking

Figure 1.3 Salkovskis' cognitive analysis of OCD (from Salkovskis, et al., 2000. p 349)

Figure 1.4 The sequence of descriptions, interpretations and actions from the Misinterpretation of Significance Theory (from Rachman, 1997, pp. 795)

Figure 2.1 Relationships between intrusive thoughts, responsibility, thought suppression and obsessive-compulsive symptoms (from Smari \& Holmsteinsson, 2001, pp. 15)

Figure 2.2 Outcome Measurement Model Study 1 30

Figure 2.3 Predictor Measurement Model Study 1 30

Figure 2.4 Inflated Responsibility (IR): Full mediation model with standardised regression weights 32

Figure 2.5 Inflated Responsibility (IR): Partial mediation model with standardised regression weights 32

Figure 2.6 Inflated Responsibility (IR): Nested model with standardised regression weights

Figure 2.7 Thought-Action Fusion (TAF): Full mediation model with standardised regression weights

Figure 2.8 Thought-Action Fusion (TAF): Partial mediation model with standardised regression weights

Figure 2.9 Thought-Action Fusion (TAF): Nested model with standardised regression weights

Figure 2.10 Thought-Action Fusion (TAF): Mixed model of moral and likelihood subscales with standardised regression weights 35 
Figure 3.1 Interaction between Inflated Responsibility and external Locus of Control in the prediction of Obsessive Compulsive Symptoms .... 46

Figure 3.2 A mediational model, with External locus of control mediating between thought-action fusion scores and OC symptoms

Figure 3.3 A mediational model, with External locus of control mediating between TAF-Likelihood scores and OC symptoms

Figure 4.1 A mediational model, with Inflated Responsibility mediating between PIRBS scores and OC symptoms 65

Figure 4.2 A mediational model, with Inflated Responsibility mediating between PIRBS scores and OC symptoms (clinical sample)

Figure 4.3 A (non significant) mediational model, with TAF mediating between PIRBS scores and OC symptoms

Figure 4.4 A mediational model, with TAF-likelihood mediating between PIRBS scores and OC symptoms

Figure 5.1 Differences between Christians and Atheists on TAF, TAF-moral and TAF-likelihood. TAF total and TAF-moral differences are significant 86

Figure 5.2 Religiosity as a moderator for TAF-Moral on OC symptoms ..... 87

Figure 5.3 Religiosity as a moderator for TAF-Moral on Depression 87 


\section{List of Tables}

Table 2.1 Means and standard deviations of latent variables Study 1

Table 2.2 Pearson correlations among latent variables with likelihood and moral TAF subscales Study 1

Table 2.3 Pearson correlations among latent variables controlling for ObsessiveCompulsive symptoms Study 1 .......................... 28

Table 2.4 Fit indices for Measurement Models Study 1 ................. 31

Table $2.5 \quad$ Inflated Responsibility: Fit indices for covariance structure analyses Study 1

Table 2.6 Thought-Action Fusion: Fit indices for covariance structure analyses

Study 1

Table 3.1 Means, standard deviations and Cronbach's alpha Study 2A .... 44

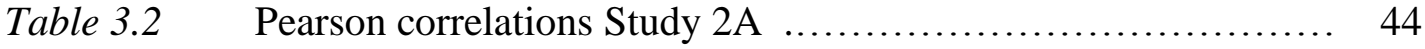

Table 3.3 The results of the multiple regression analyses Study 2A ....... 45

Table 3.4 Means, standard deviations and Cronbach's alpha Study 2B ..... 48

Table $3.5 \quad$ Pearson correlations Study 2B ............................... 49

Table 3.6 The results of the multiple regression analyses: Inflated Responsibility

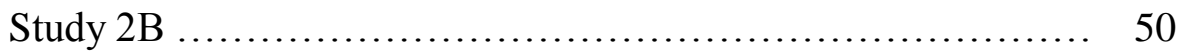

Table 3.7 The results of the multiple regression analyses: Thought-Action Fusion

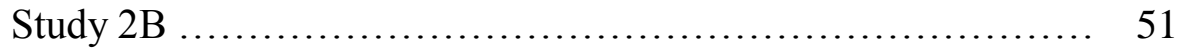

Table 3.8 The results of the multiple regression analyses: TAF-likelihood Study 2B

Table 3.9 The results of the multiple regression analyses: TAF-moral

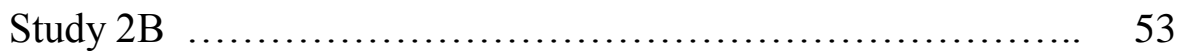

Table 4.1 Means, standard deviations and reliability Study 3A f......... 63

Table $4.2 \quad$ Pearson correlations Study 3A .............................. 64

Table 4.3 Individual Regression Analyses: Four subscales of the PIRBS Study

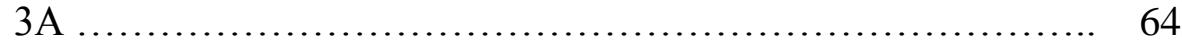

Table 4.4 Means, standard deviations and reliability Study 3B ........... 68

Table $4.5 \quad$ Pearson correlations Study 3B .............................. 69 
Table 4.6 Individual Regression Analyses: Four subscales of the PIRBS on

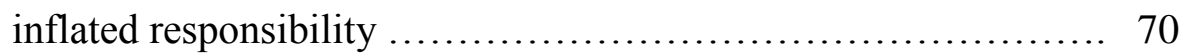

Table 4.7 Individual Regression Analyses: Four subscales of the PIRBS on Thought-Action Fusion ................................. 71

Table 4.8 Individual Regression Analyses: Four subscales of the PIRBS on Thought-Action Fusion-Likelihood ....................... 72

Table 4.9 Individual Regression Analyses: Four subscales of the PIRBS on Thought-Action Fusion-Moral ............................ 73

Table 5.1 Mean scores (and standard deviations) and reliability analyses Study

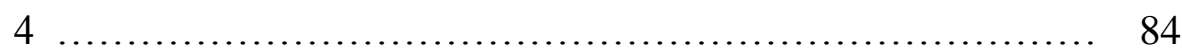

Table 5.2 Pearson correlations Study 5 ........................... 85 


\section{List of Appendices}

Appendix A

Study 1 materials.

113

Appendix B

Study $2 \mathrm{~A}$ and $3 \mathrm{~A}$ materials

115

Appendix $\mathrm{C}$

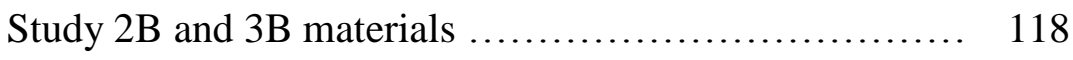

Appendix D

Study 4 materials 121

Appendix E

Obsessive Compulsive Inventory - Revised 125

Appendix F

Responsibility Attitude Scale 126

Appendix G

White Bear Suppression Inventory 128

Appendix $\mathrm{H}$

Thought-Action Fusion Scale 129

Appendix I

Self-Rating Depression Scale 130

Appendix $\mathbf{J}$

Self-Rating Anxiety Scale 131

Appendix K

Pathways to Inflated Responsibility Scale 132

Appendix L

Levenson Multidimensional Locus of Control Inventory .. 134 


\section{Preface}

Over the last decade there has been an increasing emphasis on the power of thought in our lives, and the ability to change events using only thoughts. There are numerous examples in everyday life, for example 'thinking positively' is now a widely accepted piece of advice, and it is assumed that this will make some difference to the outcome. In 2006 the self-help book 'The Secret' by Rhonda Byrne became a best seller, sold more than 19 million copies, was translated into 46 languages, and was a repeat feature on the Oprah Winfrey Show. The underlying premise of this book was that positive thinking leads to increased wealth, health and happiness. It described the law of attraction in this sense, is that if we send positive thoughts into the universe, we will receive positivity back to us, even to the point of curing cancer and overcoming obesity through the power of thought.

What happens however, when the thoughts are not so positive? While there has been a lot of attention on the power of thoughts for positive events, many people battle negative, intrusive thoughts, leading to anxiety and depression. While most people experience negative cognitive intrusions (Salkovskis, 1985, 1996), it is how we react to and process these that can make the difference between brushing them off as nothing more than thoughts, or developing severe anxiety and depression. Two ways of processing negative thoughts that have been associated particularly with Obsessive Compulsive symptoms, are the Inflated Responsibility and Thought-Action Fusion biases.

This thesis presents a comprehensive investigation of Thought-Action Fusion and Inflated Responsibility in psychopathology. Chapter 1 presents a detailed literature review of both topics. Chapter 2 investigates the response of thought suppression on responsibility beliefs. Chapter 3 then examines responsibility beliefs alongside an external locus of control. Chapter 4 covers how childhood experiences and critical events in one's life can lead to cognitive biases. The work in Chapter 5 examines Thought-Action Fusion alongside religious and atheist beliefs. Finally, Chapter 6 discusses the full body of research and what value it adds to current theory, clinical implications, and directions for future research in this area. There are three overarching research foci for this thesis, namely the specific relationship between Thought-Action Fusion and Inflated Responsibility, their roles in psychopathology, and the etiology of these types of beliefs. 
"Much more surprising things can happen to anyone who, when a disagreeable or discouraged thought comes into his mind, just has the sense to remember in time and push it out by putting in an agreeable, determinedly courageous one. Two things cannot be in one place."

Frances Hodgson Burnett, The Secret Garden 


\section{CHAPTER ONE}

\section{GENERAL INTRODUCTION AND LITERATURE REVIEW \\ 1.1 Overview}

This thesis presents a collection of studies that aim to demonstrate that both inflated responsibility and thought-action fusion play significant roles in psychopathology. While both cognitive biases have been traditionally considered in relation to obsessive compulsive disorder, it is proposed that they are more general cognitive beliefs implicated in other disorders such as anxiety and depression. There are a number of theories that attempt to address how these two types of responsibility beliefs are related, but the overall picture is far from clear. The goal of the current research is to bring some clarity to this relationship and identify similarities and differences between these concepts. Additionally, inflated responsibility and thoughtaction fusion will be considered alongside other variables such as early experiences, thought suppression and locus of control to establish their roles in psychopathology.

\subsection{Thought-Action Fusion}

Thought-action fusion (TAF) is a phenomenon whereby an individual has difficulty separating intrusive thoughts and their corresponding behaviours (Marino, Lunt \& Negy, 2008). Rachman and Shafran (1999) defined TAF as "the psychological phenomenon in which the patient appears to regard the obsessional thought and the forbidden action as being morally equivalent and/or feeling that the obsessional thought increases the probability of the feared event” (p. 72). TAF has since been dichotomised into moral and likelihood components. Moral TAF is the notion that unacceptable cognitive intrusions about disturbing events are morally equivalent to the actual occurrence of these events (Abramowitz, Whiteside, Lynam \& Kalsy, 2003). For example, Rachman and Shafran (1999) describes the case of a religious woman who experienced intrusive sexual images of Jesus while praying. This led the woman to believe that she by having such an image, had sinned and was therefore an immoral person. Likelihood TAF refers to the idea that thinking about a distressing event causes that event to become more probable. Likelihood TAF is further divided into likelihood-self where the individual believes their thoughts increase the probability that a distressing event will happen to themselves. For example if I think about being in a car accident, this makes it more likely to happen to me', and likelihood-other where the individual believes their thoughts will increase the 
probability of negative events occurring to other people, for example 'if I think about my best friend being in a car accident, it is more likely that they will be in an accident' (Abramowitz, et al., 2003).

The term 'thought-action fusion' is relatively recent; however long before its conceptualisation, clinicians had identified these characteristics in patients. For example, Bleuler (1934) described patients who feared "that they might destroy their beloved ones through a thought" and referred to this as 'omnipotence of thought' ( $p$. 561). Similar concepts of magic and magical thinking have also long been used by anthropologists, writers and psychoanalysts (Berle \& Starcevic, 2005). The early concept of TAF arose from observations of patients with obsessional thinking, who assumed that their thoughts were equivalent to actions (Salkovskis, 1985). This led on to the first study investigating the link between perceived responsibility and TAF in relation to obsessionality, which found TAF to be significantly correlated with OCD (Rachman, Thordarson, Shafran \& Woody, 1995). The development of a TAF scale came shortly after, in an attempt to be able to measure and assess TAF in a systematic manner (Shafran, Thordarson \& Rachman, 1996; Shafran \& Rachman, 2004).

TAF exists on a continuum rather than being represented as a categorical construct, with many non-clinical individuals endorsing TAF statements typically to a lesser extent than clinical participants (Rassin, 2001). While clinical samples tend to score significantly higher on measures of TAF, some overlap between clinical and non-clinical samples has been found (e.g., Abramowitz et al., 2003). Berle and Starcevic (2005) suggest a number of dimensions within TAF which can influence this, including: strength of belief, degree of insight, degree of distress caused by TAF, and strength of urge to neutralise the effects of TAF.

TAF has been studied experimentally in the laboratory examining the relationship between the frequency of thought intrusions and the distress caused by them. One example of this research is that by Rassin, Merckelbach, Muris and Spaan (1999) using university students as participants, who were told that if they thought of the word 'apple', another participant would receive an electric shock in a separate room (which did not really happen). The students were connected to EEG electrodes and told that this would allow the researchers to see when they thought of the word 'apple' and 'administer the electric shock'. This was compared to a control condition, where there was no threat of electric shock to another participant. The results demonstrated that the experiment effectively produced an actual TAF-likelihood- 
others condition such that one's thoughts did directly affect another person. Compared to the control condition, those participants in the experimental condition reported significantly more thoughts of the word 'apple', as well as more discomfort, more self-directed anger, and more resistance to thinking the word 'apple'. The researchers of this study concluded that TAF may play a role in increasing the frequency and distress of intrusive thoughts leading to significant distress.

\subsection{Inflated Responsibility}

If a person believes that his or her thoughts contribute to increasing the probability that a negative event will happen (i.e., the likelihood component of TAF), then they are likely to perceive themselves to be at least partially responsible for this. Additionally, the individual may consider it their direct responsibility to prevent this from occurring, for example by engaging in neutralising behaviours (Shafran, Thordarson \& Rachman, 1996). It has been found in previous research that those individuals who demonstrate high levels of TAF are likely to also experience a higher level of responsibility because of these dysfunctional beliefs (Shafran, Thordarson \& Rachman, 1996). Responsibility in this case, which characterises obsessional problems, can be defined as:

"the belief that one has the power which is pivotal to bring about or prevent subjectively crucial negative outcomes. These outcomes are perceived as essential to prevent. They may be actual, that is having consequences in the real world, and/or at a moral level" (Salkovskis et al., 1996; cited in Salkovskis et al., 1999, pp. 1058).

In this way, the individual believes that they will be responsible for harm (to themselves or a loved one) unless they take action to prevent it, and is considered to be an internal source of inflation of responsibility (Shafran, et al., 1996). This is different from an external sense of inflated responsibility which is more likely to arise from sources outside of the individual (e.g., door locks, knives and bacteria) which commonly lead to compulsive checking and washing. However, both internal and external triggers are similar in that they both produce the urge to take action to prevent the event occurring (Rachman, 1993). TAF and also this inflated sense of responsibility are considered to be fundamental components of the catastrophic misinterpretation of intrusive thoughts (Shafran et al., 1996). 
Berle and Starcevic (2005) argued that the responsibility beliefs experienced by people with intrusive thoughts take one of two forms. The first of these is the feeling of being responsible for harm prevention. That is, after the occurrence of an intrusive thought, the individual feels personally responsible for preventing the negative event from actually happening. The second type of belief is the perceived responsibility for the existence of the negative thoughts themselves. This represents the individual's feelings of moral responsibility and attribution of great significance for having the thoughts themselves.

\subsection{Relationship between the two}

Despite being theoretically connected, the particular relationship between TAF and inflated responsibility remains unclear. Clarity on this has not been helped by the ever changing definitions of each construct. For example, Berle and Starcevich (2005) describe the ongoing debate as to whether inflated responsibility refers to specific situations, or is a more general attitude. Researchers (Rassin et al., 1999; Shafran et al., 1996) have described TAF as being closely related to inflated responsibility, but also distinct. This related-but-separable perspective is supported by numerous studies demonstrating only moderate correlations between the two variables (e.g. Gwilliam, Wells, \& Cartwright-Hatton, 2004; Rachman, et al., 1996; Smari \& Holmsteinsson, 2001; Yorulmaz, Yilmazl \& Gencoz, 2004). What is clear, at least, is that TAF and inflated responsibility do not represent completely overlapping concepts. This leaves at least two plausible options: (A) There is some conceptual overlap and they may cooccur, but the presence of one neither enhances nor inhibits the presence of the other, or; (B) TAF is a subordinate or specific type subsumed under the more superordinate concept of inflated responsibility. In this subordinate/superordinate relationship, TAF cannot occur in the absence of inflated responsibility (see Figure 1.1). Based on the extant research, the former relationship seems more likely than the latter.

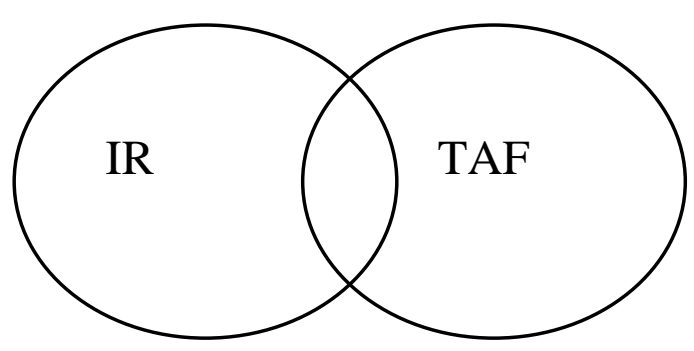

(A)

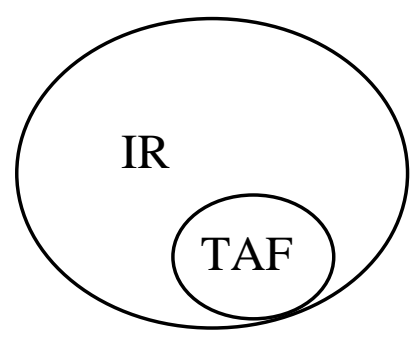

(B)

Figure 1.1 Inflated Responsibility (IR) and Thought-Action Fusion (TAF) 
Some research suggests that TAF can lead to inflated responsibility (e.g. Rachman, 1993; Rachman et al., 1995; Rassin, Merckelback, Muris \& Spaan, 1999; Shafran et al., 1996). Clark (2004) proposes that TAF inflates responsibility, especially in the presence of aggressive, blasphemous and sexually themed intrusive thoughts. Shafran, Thordarson and Rachman (1996) described TAF as a possible cause or antecedent of an inflated sense of responsibility, which plays an important role in the transition of normal thoughts into clinical obsessions. That is, patients with the TAF bias are prone to inflated responsibility because of their TAF beliefs, which, in turn lead to increased feelings of guilt (Rachman, 1993; Shafran et al., 1996). Additionally, as well as being a product of TAF, inflated responsibility may also contribute to the occurrence of TAF, this way representing both an antecedent and consequence of the TAF bias in a mutually reinforcing relationship (Rachman, 1997; Clark, 2004).

Salkovskis (1985) suggests that individuals with TAF believe there is a link between their thoughts and the event occurring, leading to the need for action to prevent this happening, indicating that the person feels responsible for negative events unless they take appropriate action to prevent them. Having an inflated sense of responsibility can contribute both to the development of TAF, and may also be one of the effects of TAF (Rachman, 1997; Clark, 2004). A further consequence of this inflated responsibility is the need for mental neutralising; for example, reconstructing the intrusive thought or image such that the people involved are no longer harmed or injured. This form of neutralising can also be interpreted as a form of TAF, i.e. the individual believes they can influence the likelihood of events by changing their thoughts or images (Shafran, Thordarson \& Rachman, 1996).

Shafran et al. (1996) showed that likelihood TAF has a higher correlation with responsibility than moral TAF; TAF moral is not as directly linked to responsibility possibly because there is no harm for the patient to prevent. Shafran et al. further describe TAF as being closely related to, but distinct from, responsibility whereas Rachman (1997) posits the theory that TAF is a 'variation' of inflated responsibility. There is, however, limited evidence to support this claim, with previous studies showing TAF to be only moderately correlated with responsibility (Berle \& Starcevic, 2005). TAF likelihood appears to have a closer association with responsibility compared with TAF moral, especially responsibility for preventing harm, both to others and the self (Berle \& Starcevic, 2005). Throughout previous research, measures 
of overall TAF beliefs have typically shown only moderate positive correlations with measures of responsibility (e.g. Rachman, Shafran, Mitchell, Trant \& Teachman, 1996; Smari \& Holmsteinsson, 2001), with one study by Rachman et al. (1996) reporting no significant correlation between TAF moral and responsibility beliefs

\subsection{Magical Thinking}

While the precise relationship between TAF and inflated responsibility remains unclear, this is further complicated by magical thinking. Magical thinking refers to the belief in the personal power to control events beyond culturally accepted laws of causality (Woolley, 1997). This does not necessarily mean that there is an influence of thoughts upon events (Berle \& Starcevich, 2005). Moulding and Kyrios (2006) suggest that magical thinking can increase one's sense of control and provide a way of coping for people under stress. Interestingly, Einstein and Menzies (2004a, 2004b) propose that TAF is a specific type of magical thinking. This was based on their research with both clinical and undergraduate samples that found that TAF beliefs were only related to OC symptoms through magical ideation. Specifically, Einstein and Menzies suggest that the TAF likelihood component is a type of magical thinking, whereas the TAF moral component is not so closely related (though perhaps more aptly related to other variables such as religiosity). This may be because TAF likelihood is more like magical thinking in that thoughts are assumed to have causal influence over events and probability of their occurrence (Berle \& Starcevich). Figure 1.2 represents the theoretical relationship between Inflated Responsibility (IR), Thought-Action Fusion (TAF), and Magical Thinking. Although TAF is subordinate within the more superordinate concept of magical thinking, it remains related, but separate from inflated responsibility.

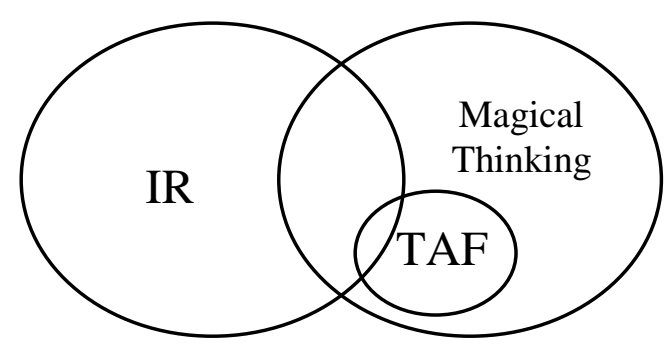

(C)

Figure 1.2 Inflated Responsibility (IR), Thought-Action Fusion (TAF), and Magical Thinking 


\subsection{Obsessive Compulsive Disorder (OCD)}

Not surprisingly, TAF and inflated responsibility are often referred to in the context of their occurrence in Obsessive Compulsive Disorder (OCD). OCD is a debilitating characterised by intrusive, disturbing thoughts/obsessions, and repetitive, compulsive behaviours (Chamberlain, Blackwell, Fineberg, Robbins \& Sahakian, 2005). The obsessions of OCD typically involve distressing thoughts of contamination, socially unacceptable thoughts and behaviours, and harm or death of a loved one. They can also involve a preoccupation with counting and symmetry (Chamberlain, et al., 2005). Compulsions can include behaviours such as excessive hand washing, making objects symmetrical and repeated checking (e.g. light switches and door locks; Chamberlain, et al., 2005).

\subsubsection{Obsessive Compulsive Disorder and TAF}

Previous studies have repeatedly demonstrated a relationship between TAF and OCD, with stronger positive correlations for TAF-likelihood than for TAF-moral (Amir, et al., 2001). TAF has been described as being a contributing factor in the maintenance and development of OCD for two main reasons. First, the belief that thoughts are the moral equivalent of their occurrence is likely to cause the individual to become distressed (Abramowitz, et al., 2003). Second, as a consequence of the belief that their thoughts will increase the probability of an unwanted event occurring, OCD patients may engage in certain behaviours and compulsions in order to prevent these negative events from occurring (Abramowitz, et al., 2003).

Research by Amir, Freshman, Ramsey, Neary and Brigidi (2001) showed individuals with OC symptoms were more likely to endorse beliefs that the likelihood of negative events happening was a result of their thoughts. They also found that there were no significant differences between OC participants and healthy controls on measures tapping the moral component of TAF. Similar research has also found anxiety to be specifically related to the TAF likelihood component, whereas the TAF moral component correlates more strongly with measures of depression. Studies such as these have led many to believe that the likelihood component has a closer relationship with OCD than the moral component of TAF, which tends to become significant only when mediated by other variables, for example depression and religiosity (Coles, Mennin \& Heimberg, 2001). 


\subsubsection{Obsessive Compulsive Disorder and Inflated Responsibility}

Inflated responsibility also plays a significant role in OCD (Rachman, Thordarson, Shafran \& Woody, 1995). As Salkovskis and Kirk (1989) describe, obsessional thought often involves some fear of personal responsibility for preventing harm, either to the self or others. Many experimental and correlational studies support the role of responsibility beliefs in OCD (e.g. Rachman, Thordarson, Shafran \& Woody, 1995; Smari \& Holmsteinsson, 2001; Yorulmaz, Altin \& Karanci, 2008). One example is of Lopatka and Rachman (1995) who used an experimental design to manipulate feelings of perceived personal responsibility for a negative event in patients with OCD. The results showed that participants showed a decrease in discomfort and the need to neutralise after a manipulation leading to a reduction in their responsibility beliefs. Conversely, neutralising attempts and feelings of discomfort increased following a manipulation which induced inflated responsibility. The changes in overall discomfort were not found to be associated with the person's sense of control over the activity. Similar findings were shown in a study by Rachman (1993), where it was shown that OCD patients' neutralising symptoms and feelings of discomfort decreased upon first being admitted at a hospital, and returned a few days after arriving at the new environment. Rachman interpreted this finding as a demonstration of inflated responsibility - when patients first arrived at the hospital, they felt less in control (therefore less personally responsible); but over time they developed a sense of belonging and began to feel more responsible towards their surroundings.

Rachman (2002) discussed the role of inflated responsibility, in combination with other variables, in increasing the intensity and duration of neutralising behaviours. He proposed that the most destructive combination is of high levels of inflated responsibility alongside high probability of harm and estimation of serious costs (Altin \& Karanci, 2008). The importance of inflated responsibility in the development and maintenance of OCD is evident in cognitive models, for example Salkovskis' (1996) cognitive model of OCD where responsibility beliefs play a pivotal role.

\subsection{Cognitive Models}

\subsubsection{Salkovskis' Cognitive Model of OCD}

Salkovskis' $(1985,1996)$ influential model of obsessions describes how unwanted intrusive thoughts are a common part of everyday life and occur on the 
'normal' end of a continuum of clinical obsessions. These thoughts may escalate into obsessions when they are misinterpreted in terms of their potential to cause harm, and the responsibility the individual takes for these thoughts. Intrusive thoughts, therefore, are not limited to people with OCD. In their seminal study, Rachman and De Silva (1978) found that the classic content of obsessions seen in OCD is also regularly experienced by the majority of the general population. However what causes these intrusions to become clinical obsessions is that they are experienced with greater intensity and frequency, eliciting significant discomfort and the desire to resist them (Rassin, 2001).

Salkovskis (1996) argues that this belief of personal responsibility for preventing harm either to the self or others results in overwhelming anxiety. In reaction to this, the individual will direct their attentional efforts towards removing the cognitive intrusion, and therefore decrease the sense of responsibility. However, as Salkovskis describes, the following outcomes are more likely: (a) increased anxiety, discomfort and depression; (b) cognitive intrusions becoming more significant and more accessible; (c) the individual becoming more focused on the intrusion, paying more attention to it; and (d) the individual beginning to use neutralising techniques in an effort to reduce anxiety and discomfort (see Figure 1.3). To summarise, this cognitive model of OCD proposes that symptoms are caused by beliefs of inflated responsibility, and are maintained by the individuals' responses to this, by trying to be sure they have not caused harm (Salkovskis, 1999; Salkovskis, Thorpe, Wahl, Wroe \& Forrester, 2003). 


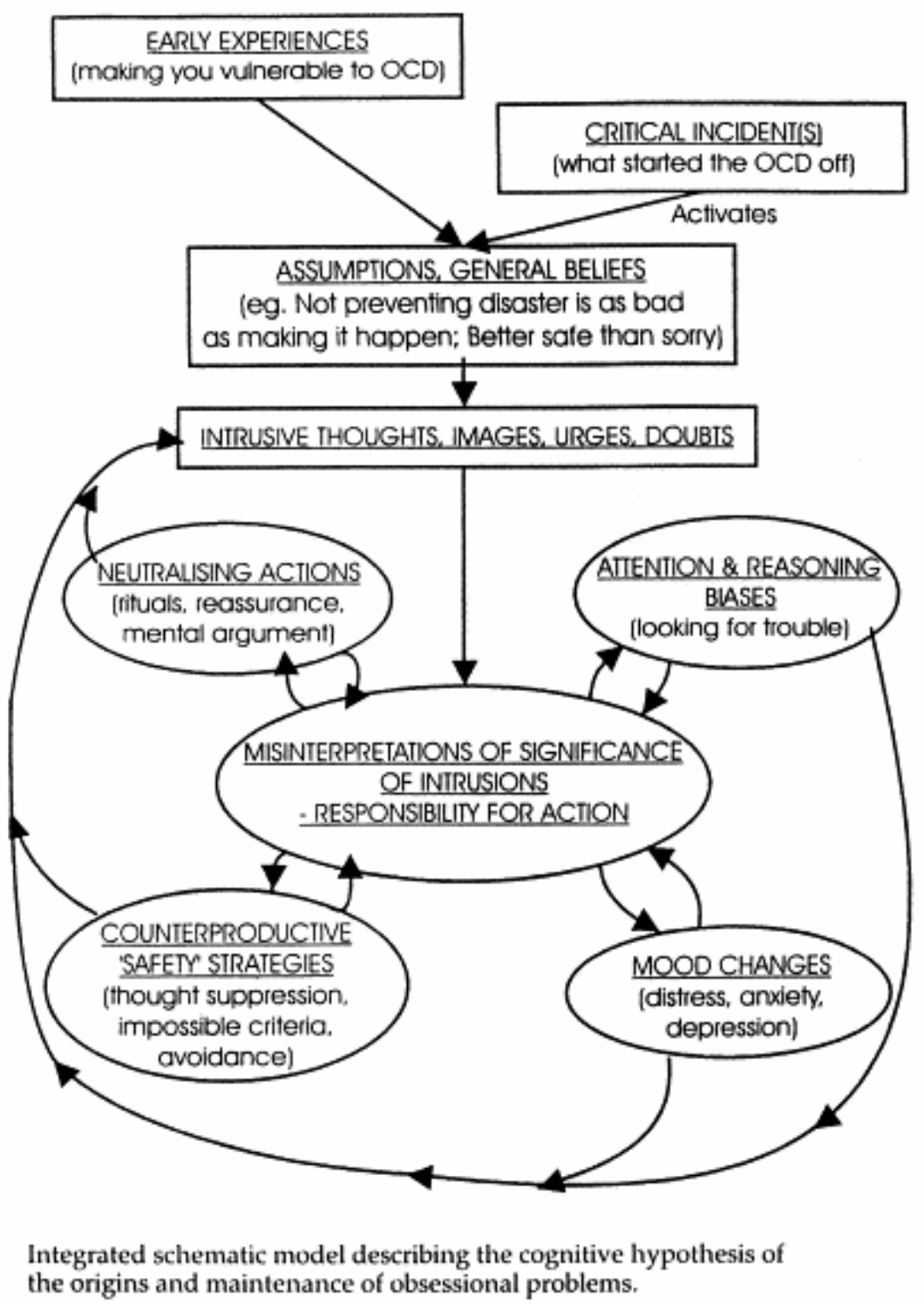

Figure 1. 3 Salkovskis' cognitive analysis of OCD (cited in Salkovskis et al., 2000.p 349.)

\subsubsection{Neutralising}

Salkovskis $(1989$, p. 678) describes a neutralising response as a "voluntarily initiated activity which is intended to have the effect of reducing perceived responsibility". This response can be either psychological (e.g. thought suppression) or behavioural (e.g. checking), and usually becomes repetitive, and is very successful for reducing anxiety in the short term. In the long term, neutralising responses are counter-productive as they prevent the individual from addressing their intrusive thoughts and processing evidence that would disconfirm their beliefs of personal responsibility. In essence, neutralising techniques serve to increase overall discomfort and anxiety. This is because neutralising (a) increases the individual's preoccupation with the negative intrusion; (b) is unsuccessful in the long term, leading to increased 
frequency of the intrusion; (c) increases the salience and perceived importance of the intrusion; and (d) prevents the individual processing the intrusion and discontinuing the belief that thoughts are harmful (Salkovskis \& Forrester, 2002).

\subsubsection{Misinterpretation of Significance Theory}

Rachman $(1997 ; 1998)$ proposed a cognitive theory of obsessions examining how they are formed and persist over time. The central assumption of this theory is that obsessions are "caused by catastrophic misinterpretations of the significance of one's intrusive thoughts/images/impulses" (Rachman, 1998, p. 385). Rachman (1997) proposed that obsessions persisted as long as the misinterpretations continued, and would disappear or at least deteriorate if the misinterpretations were weakened or eliminated. These 'catastrophic misinterpretations' involve the individual having the belief that their intrusive thoughts are signs or indications of something meaningful and significant about their character, which then leads to negative consequences (Clark, 2004). Rachman (1997) went on to further define misinterpretations of significance and how these can be measured on five dimensions, as cited in Clark (2004):

1. Importance. The intrusive thought is not viewed as meaningless or trivial, but reveals something important about the individual.

2. Personalised. The significance of the thought is interpreted as being personalised and of importance to the individual in particular.

3. Ego-alien. The content of the intrusive thought is ego-dystonic, and is considered to be uncharacteristic and unlike the individual.

4. Potential consequences. The individual believes the cognitive intrusion to have potential consequences, and is not merely a passing thought.

5. Serious consequences. The potential consequences of the intrusion are viewed as being serious, typically involving a degree of threat, harm or danger.

Through misinterpreting cognitive intrusions, the thought becomes important, personally significant, revealing, threatening and catastrophic, all of which transform a normal thought into an obsession and a torment for the affected individual (Rachman, 1997). For example, Rachman describes the case of a 25 year old male computer analyst who reported having repeated thoughts and images of hurting the young children of a friend. He interpreted this as meaning he was a potential 
murderer, and an evil and worthless human being. Another example is that of an affectionate grandmother who experienced recurrent images of throwing her grandson from a balcony, which caused deep distress and even suicidality, leading her to believe she was a dangerous psychopath incapable of love for other people (Rachman, 1997). Not surprisingly, individuals who are affected by intrusions such as this, often experience a strong urge to cancel or atone for the obsession, referred to as neutralisation attempts (Rachman, 1997). The neutralising response includes any voluntary activity performed to reduce perceived responsibility and results in a temporary reduction in the discomfort caused by the intrusive thought (Salkovskis, 1989). An example of neutralisation has been described by Rachman as that of a female patient who reported having recurrent thoughts and images of stabbing her own children. The patient then engaged in avoidance behaviours by avoiding all contact with sharp objects, and eventually installing strong locks on her kitchen doors, only entering while accompanied by a trusted adult.

Compulsions such as checking and washing strongly resemble neutralisation and avoidance strategies in that they serve the same psychological function of providing some relief of the distress and discomfort caused by the obsessions (Rachman, 1997). Neutralisation, avoidance and compulsions persist over time because they succeed at reducing (although temporarily) the distress caused by the disturbing thoughts and images. This learned relationship, however, acts to preserve the pathway between obsessions, misinterpretations and their consequences, as illustrated in Figure 1.4 (Rachman, 1997). Avoidance strategies may also serve to increase the frequency of unwanted intrusions (Rachman, 1998). The avoidance of anxiety-inducing stimuli, and the resulting decrease in sensations of anxiety, reinforces the belief that exposure to those stimuli leads to the feeling of distress and loss of control (Clark, 2004). Additionally, the repeated pattern of using avoidance strategies prevents exposure to evidence that would disconfirm the individual's beliefs in the danger and potential negative consequences of their thoughts (Clark, 2004; Rachman, 1998, 2003). Additionally, the increase in negative intrusive thoughts provides further evidence of the apparent significance of the intrusion (Clark, 2004). 


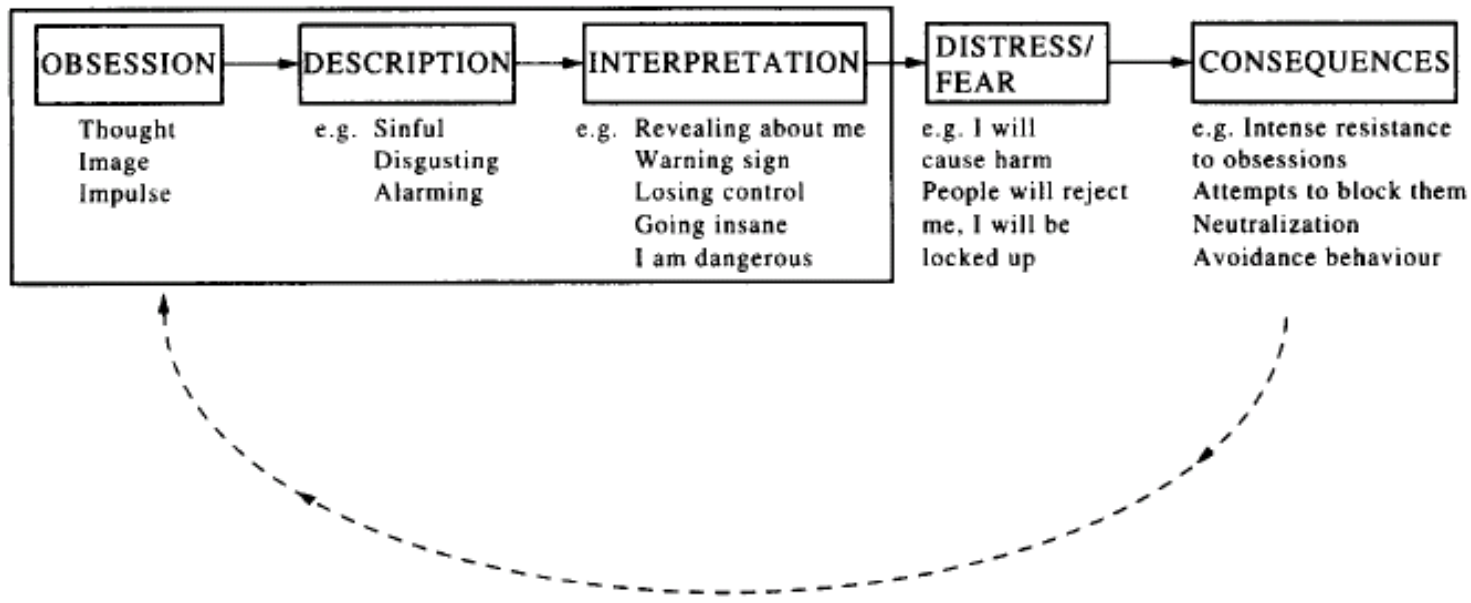

Figure 1.4 The sequence of descriptions, interpretations and actions from the Misinterpretation of Significance Theory (from Rachman, 1997, pp. 795).

In relation to this cognitive model, TAF is considered to be a cognitive bias which sets up a vulnerability for obsessionality. In other words individuals who demonstrate a TAF bias may be more likely to make negative, catastrophic misinterpretations of significance in response to intrusive thoughts (Clark, 2004). More specifically, TAF may represent a vulnerability for individuals to make incorrect appraisals of personal excessive responsibility about cognitive intrusions (Clark, 2004). Rassin, Merckelbach, Muris and Spaan (1999) describe how those individuals who demonstrate this inflated sense of responsibility in reaction to intrusive thoughts, experience more discomfort when these thoughts occur, placing them at greater risk for developing clinical obsessions.

\subsection{TAF and Inflated Responsibility in other psychopathology}

TAF and inflated responsibility beliefs may not be confined to OCD. Associations have been found with a number of other disorders, including depression, eating disorders and anxiety disorders (Berle \& Starcevic, 2005).

\subsubsection{Other Anxiety Disorders}

Rachman and Shafran (1999) have described how TAF can manifest in anxiety disorders other than OCD. For example, they describe how people with social anxiety may believe that their unkind thoughts increase their chance of rejection, how people with panic attacks may believe that thinking about catastrophic events and losing control may increase the chance of it happening, and how people with posttraumatic stress disorder may believe that thinking about the trauma may increase the chance of it happening again. This has been supported by empirical evidence, with 
studies showing no differences in scores of TAF between groups of OCD patients and those with other anxiety disorders including panic disorder, social anxiety and posttraumatic stress disorder (Rassin et al., 2001). Interestingly, although TAF and inflated responsibility beliefs are evident in a range of anxiety disorders, the individuals' responses to these may differ. Berle and Starcevich (2005) suggest that while OCD patients will engage in various neutralising techniques in an attempt to reduce discomfort, individuals with other anxiety disorders with rely on avoidance strategies alone.

TAF and inflated responsibility beliefs have also been found to be important in the development and maintenance of pathological worry, as seen in generalised anxiety disorder (GAD; Hazlett-Stevens, Zucker \& Craske, 2002). TAF likelihood in particular seems to be very similar to beliefs held by individuals with GAD about their worry causing the occurrence of feared events occurring. Hazlett-Stevens et al. investigated this with a sample of 494 undergraduate students, measuring TAF and pathological worry. Results indicated that while those students who endorsed high levels of worry (also diagnostic for GAD) also scored significantly higher on the TAF likelihood subscale, there were no significant results for the TAF moral subscale.

\subsubsection{Depression}

It has been suggested that depression can possibly be the product of combining TAF, inflated responsibility and guilt (Rachman \& Shafran, 1999). There has been a lot of research implicating TAF beliefs in depression, particularly the moral component of TAF. For example, Abramowitz et al. (2003) found TAF moral component scores to be significantly correlated with scores on the Beck Depression Inventory (BDI; Beck \& Steer, 1987) when controlling for anxiety, but no association with the TAF likelihood component (which did correlate significantly with measures of anxiety). Abramowitz et al. speculated that unlike TAF-likelihood which seems more related to anxiety, TAF-moral is more directly related to depressive tendencies through self-blame and guilt which are common depression symptoms.

\subsubsection{Eating Disorders}

Individuals with eating disorders often have 'magical' type beliefs about food and their weight similar to cognitive distortions of TAF (Garner \& Bemis, 1982). Rachman and Rachman (1999) described this variation as 'Thought-Shape Fusion' (TSF), where people who are excessively concerned about their weight and body image form connections between negative intrusive thoughts and their body shape. 
According to Rachman and Rachman, TSF has three underlying beliefs: (a) thinking that eating 'forbidden' food increases the likelihood of gaining weight; (b) thinking about eating 'forbidden' food is morally equivalent to actually eating it; and (c) thoughts about eating 'forbidden' foods increases the perception of fatness.

Shafran et al. (1999) assessed TSF in a sample of 119 undergraduate students, and found it to be significantly associated with eating disorder psychopathology. Also, results showed TAF and TSF to be significantly correlated, although the authors postulate that the two are distinct. Where TAF is considered to be a cognitive bias, TSF also involves distortions of perception (Berle \& Starcevic, 2005). In a similar study, Shafran and Robinson (2004) compared a group of 42 women with clinical eating disorders with a group of 42 healthy controls and found TSF to be significantly associated with eating disorders, when controlling for depression.

\subsubsection{Psychotic Disorders}

An important distinction must be made when discussing TAF and responsibility beliefs in terms of psychosis. With disorders such as anxiety and depression, there remains full insight that TAF is irrational and that thoughts alone cannot influence events. The same cannot be said for individuals suffering from psychosis as there are some similarities between such beliefs and aspects of psychosis (Rachman \& Rachman, 1999). For example, individuals with delusions can engage in magical thinking and form links between events. Also, thought broadcasting delusions can involve the individual believing that their thoughts can be seen and read by others and influence external events (Berle \& Starcevich, 2005). Examples can also be seen in grandiose delusions, with individuals believing their thoughts have magical powers, and ideas of reference where someone may think that an event is related to something they have been thinking about (Berle \& Starcevic).

Based on previous research, magical thinking seems to mediate the relationship between psychotic disorders and TAF and responsibility beliefs. An example of this is shown by Lee, Cougle and Telch (2005) who reported TAF likelihood to be significantly correlated with measures of schizotypy. However, after controlling for depression, anxiety and OCD, TAF likelihood was only related to the magical ideation subscale of the schizotypy measure. While there may be evidence of TAF and responsibility type beliefs in psychotic disorders, it is important to remember the difference between these and other disorders in terms of insight, which in turn has implications for the ability to effectively treat. 


\subsection{Religiosity}

One theory as to why TAF beliefs may develop is that by Salkovskis et al. (1999), who hypothesised that an individual may come to have TAF-moral beliefs because of belief systems with strict behavioural and moral codes, which have been imbued on them by an authority figure (e.g., school teacher or religious leader). It may not be surprising therefore, that religiosity has been linked with TAF, typically showing a stronger relationship between religiosity and TAF-moral, rather than with TAF-likelihood (Rassin \& Koster, 2003). Salkovskis et al., (1999) describe how the interpretation of religious teachings can trigger obsessional problems such as "sin by thought" (an example of TAF-moral). That fact that TAF-likelihood is not related to religiosity may be because it is more a type of magical thinking, in that thoughts are assumed to have causal influence over events and probability of occurrence (Berle \& Starcevich). This suggests a kind of superstition along the lines of 'tempting fate', a belief not commonly held by those who believe in Divine intervention and predeterminism.

It is important to note that it is not necessarily religious teachings that cause the development of TAF; it may be that those individuals who are predisposed to having TAF beliefs are more likely to have religious beliefs also (Rassin \& Koster, 2003; Berle \& Starcevic, 2005). Moreover, the individual's interpretation of their belief system, their personal conviction, and/or fear of punishment may be more important in the correlation between TAF beliefs and religiosity (Berle \& Starcevic, 2005). The extreme nature of some kinds of teachings may also play a role (Salkovskis et al., 1999). Marino, Lunt and Negy (2008) found that those individuals with unusually rigid religious beliefs were more likely to feel responsible for their negative intrusions. These cognitions and inflated responsibility were found to occur across a range of situations and contexts in everyday life, leading them to experience unnecessary distress.

Shafran et al. (1996) illustrate the possible role of religious teachings in the development of TAF; particularly that it is sinful and wicked to have aggressive and blasphemous thoughts. Many religious teachings are related to the concept of morality; for example, the often cited passage from the Bible: "You have heard that it was said, "Do not commit adultery." But now I tell you: Anyone who looks at a woman and wants to possess her is guilty of committing adultery with her in his heart" (Matthew 5: 27-28; New American Standard Version). Depending on 
interpretation, this can suggest that the thought is morally equivalent with the action itself. Further evidence of the link between religion and TAF can be seen in the Thought-Action Fusion Scale (Shafran et al., 1996), with three of the 19 items referring explicitly to religion (e.g 'Having a blasphemous thought is almost as sinful to me as a blasphemous action', 'When I think about making an obscene remark or gesture in church, it is almost as sinful as actually doing it', and 'Having obscene thoughts in a church is unacceptable to me').

There is empirical evidence that suggests a link between religiosity and TAF. For example, Rassin and Koster (2003) investigated this relationship with a nonclinical sample of undergraduate university students. The results of this research demonstrated that religiosity was positively correlated with TAF, especially the moral component of TAF. It is important to note that the correlational nature of this research means that causation cannot be inferred. Therefore Rassin and Koster concluded that certain aspects of religion involve particular cognitions that are considered to be associated with obsessionality and correspond with aspects of TAF.

\subsection{Thought Suppression}

Recent research has suggested that TAF and inflated responsibility beliefs may lead normal intrusive thoughts to become pathological through the process of thought suppression. Thought suppression is one of the most common strategies people use to remove unwanted thoughts (Amir, Cashman \& Foa, 1997). It refers to an attempt to reduce/neutralise the anxiety and discomfort caused by an unwanted thought or image by intentionally trying to remove it from attention (Beevers, Wenzlaff, Hayes \& Scott, 1999). However, although it is used as an attempt to avoid the negative intrusion, thought suppression has been found to have a counterproductive effect by increasing the frequency and intensity of the intrusion, a phenomenon often referred to as the 'white bear effect' paradoxically increasing obsessive compulsive symptoms (Wegner, Schnieder, Carter \& White, 1987). The studies by Wegner, Schneider, Carter and White (1987) showed that attempts at thought suppression were most likely to result in more rather than fewer cognitive intrusions, and consequently pathological symptoms. Additionally, Wegner et al. (1987) found that this increased frequency of intrusions occurred not only at the time of attempted suppression, but also much later on, a phenomenon known as the 'rebound effect'. As Wegner (1989, p. 167) concludes: "an obsession can grow from nothing but the desire to suppress a thought". 
It has been widely suggested that the misinterpretation of the significance of intrusive thoughts through inflated responsibility beliefs and TAF appraisals may lead the individual to try to suppress these thoughts or prevent them from returning (Berle \& Starcevic, 2005; Rassin, Muris, Schmidt \& Merckelbach, 2000). Through this process, normal intrusive thoughts may become pathological (Berle \& Starcevich, 2005). Rassin et al. (2000) used structural equation modelling to examine TAF and thought suppression in relation to OCD in a sample of undergraduate students. Results indicated that TAF led to suppression attempts, which in turn resulted in higher OC symptoms. It was also found that while TAF-likelihood had a direct effect of increasing OC symptoms, TAF-moral increased symptoms only through the mediating influence of thought suppression. The authors highlighted the intermediary role of thought suppression between TAF and OC symptoms.

Berle and Starcevic (2005) suggest that intrusive thoughts, TAF and thought suppression together form a 'vicious circle', in which the individual experiences negative intrusions, which are interpreted using TAF appraisals, leading to thought suppression, which then leads to an increase in frequency and intensity of the intrusion. This is consistent with Rachman (1998) who claimed that the increased significance assigned to an unwanted thought will lead to intense efforts at suppression, which paradoxically increases the frequency of the thought, strengthening the initial misinterpretation.

\subsection{Chapter Summary}

Although they are distinct constructs, TAF and inflated responsibility share similar distortions in cognition which have been associated with a range of psychological disorders. It has been repeatedly shown that beliefs of inflated responsibility and TAF appraisals play a role in psychopathology, especially OCD. Understanding this role and where these types of beliefs come from will lead to more specific, focused and effective psychological interventions for OCD in particular, as well as other disorders. Research has shown promising results of inflated responsibility beliefs, TAF appraisals and thought suppression in their susceptibility to change through therapy. Therefore, the more we know about these, more successful and tailored treatment methods can be developed.

\subsection{The Current Research}

The aim of the current research was to conduct a comprehensive examination of thought-action fusion and inflated responsibility. The three underlying research 
aims were to provide clarity on the specific relationship between thought-action fusion and inflated responsibility, to determine their roles in psychopathology, and to provide empirical evidence for etiological theory. There exist two substantial limitations in the current research on both types of cognitive bias. Firstly, most research tends to focus specifically on links with OCD and obsessionality, without considering other disorders such as depression and anxiety. It is important to elucidate whether these types of beliefs are specific symptoms of OCD, or rather are more general cognitive biases that can be seen in other disorders. This has implications for the treatment of such disorders. Secondly, there is a lack of research with clinical samples, especially in regards to wider psychopathologies than OC symptoms. The current studies will address these issues and examine how the findings fit in with existing cognitive theory. Studies 1, 2A and 2B investigate the presence of both types of belief alongside symptoms of OCD, depression and anxiety. Study 1 uses structural equation modelling to investigate these relationships as well as exploring the use of thought suppression. Studies $2 \mathrm{~A}$ and $2 \mathrm{~B}$ examine responsibility and control beliefs with both clinical and nonclinical samples. These results will shed light on the presence of responsibility biases in a range of disorders, and how they may work alongside thought suppression and control beliefs to maintain symptoms. Studies 3A and 3B aims to provide empirical evidence for Salkovskis et al.'s (1999) theory for the etiology of inflated responsibility, using clinical and nonclinical samples. This will also be the first research to investigate whether this theory applies also to thought-action fusion. Finally, Study 4 will consider whether both types of responsibility bias are solely symptoms of psychopathology, or if they can exist without leading to distress when they are considered culturally normative. This will be investigated using Christian and Atheist samples. If results show responsibility beliefs to be unrelated to psychopathology in the Christian sample, this provides evidence for them to be considered normal in particular contexts. 


\section{CHAPTER TWO}

\section{INFLATED RESPONSIBILITY AND THOUGHT-ACTION FUSION IN PSYCHOPATHOLOGY, AND THE ROLE OF THOUGHT SUPPRESSION}

\subsection{Introduction}

Previous research has shown strong links between OC symptoms and both TAF and inflated responsibility. There has also been an interest in how these factors may be associated with other disorders such as anxiety and depression (Berle \& Starcevic, 2005), as well as various maintenance strategies (e.g. thought suppression and neutralising). Studies that examine a broader range of symptoms are important for determining how wide-ranging responsibility beliefs are - whether they are confined to OCD or are more general cognitive biases that present in a variety of psychopathologies. Thus, the goal of the current study was to build on existing research of TAF and inflated responsibility by investigating their role not only alongside OC symptoms, but also anxiety and depression, with the inclusion of thought suppression. It is proposed that responsibility beliefs are not specific to OCD, and can be seen more generally throughout other psychological disorders.

Rassin et al. (2000) used a structural equation modelling approach to investigate the relationships between TAF, thought suppression and obsessive compulsive symptoms in a non-clinical sample. They found that the TAF likelihood component had a direct effect of increasing the symptoms of OCD. The moral component however, also increased OCD symptoms, but through an indirect route involving thought suppression (Rassin et al., 2000). This is consistent with the theory that TAF-likelihood is more related to possible catastrophic consequences, and is therefore likely to lead to compulsions or avoidance as a way of preventing these, whereas TAF-moral tends to induce thought suppression rather than overt behaviours (Rassin et al., 2000). Rassin et al. argued that their findings suggested that TAF is more fundamental to causing OC symptoms than is thought suppression. The finding that thought suppression plays an intermediary role between TAF and OC symptoms lends supports for therapeutic interventions focusing on the cognitive biases of patients, thereby decreasing thought suppression attempts (Rassin et al., 2000). 
In a similar study, Smari and Holmsteinsson (2001) investigated the mediating roles of responsibility attitudes and chronic thought suppression on intrusive thoughts and obsessive-compulsive symptoms. In this study, the researchers considered TAF and responsibility attitudes as representing the same underlying construct. The participants for this research were 211 undergraduate Icelandic university students. Results demonstrated that responsibility attitudes mediated between intrusive thoughts and chronic thought suppression, and thought suppression mediated between responsibility attitudes and obsessive-compulsive symptoms. Regression analyses showed evidence of the mediating role of responsibility and thought suppression between intrusive thoughts and obsessive-compulsive symptoms (Figure 2.1), which is consistent with Salkovskis' (1996) model.

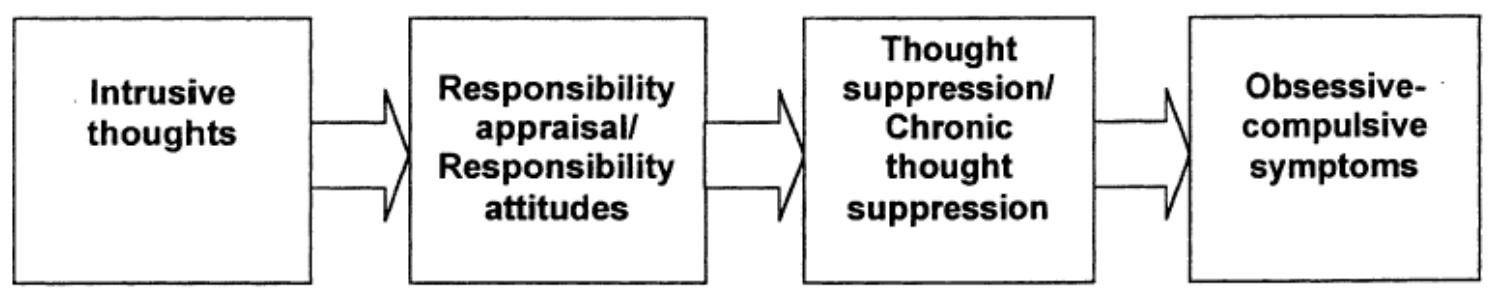

Figure 2.1 Relationships between intrusive thoughts, responsibility, thought suppression and obsessive-compulsive symptoms (from Smari \& Holmsteinsson, 2001, pp. 15).

Previous research shows a strong link between TAF and OCD, however there is a growing body of evidence that suggests TAF has implications in a wide range of psychopathology, including eating disorders, general anxiety, phobias, panic disorder, post-traumatic stress disorder, and depression (Marino, Lunt \& Negy, 2008; Berle \& Starcevic, 2005; Shafran \& Rachman, 2004). The way in which TAF is related to an inflated sense of responsibility, with OCD as a whole, and other psychological disorders is still to be determined. Berle and Starcevic (2005) suggest that to examine the role of TAF in a variety of disorders would involve the experimental manipulation and reduction of TAF and evaluating the result of these manipulations. The question of possible intervening variables in the relationships between TAF and disorders including OCD, has raised the possibility of an additional factor involved in these relationships, perhaps mediating the association between TAF and the disorders (Berle \& Starvevic, 2005). Abramowitz et al. (2003) argue that if TAF is related not only to OCD but a variety of disorders, then it is a more general cognitive bias rather 
than a specific proponent of OCD. This also supports the suggestion of an additional variable which acts as a mediator between a disorder and TAF.

Previous research has shown depression to be related to TAF in both adults and adolescents, although the correlations are usually only small to medium (Berle \& Starcevic, 2005). Abramowitz et al. (2003) compared levels of TAF, depression and anxiety in patients with OCD and non-clinical participants. Their findings showed that negative affect accounted for at least 30\% of the variation in TAF-likelihood between the OCD group and the non-clinical participants. The authors proposed that the OCD patients scored so much higher on measures of TAF because they had higher levels of depression and anxiety. Supporting this proposition, TAF was found to be associated with both anxiety and depression, supported by a significant mediation where negative affect mediated the relationship between OCD and TAF. The findings of this research showed that higher scores of TAF were related to more severe depression and anxiety. When broken down into the specific TAF components, TAFlikelihood was related to anxiety, and TAF-moral was related to depression (Abramowitz et al., 2003). This may be because TAF-moral is associated with symptoms that are evident in depression, such as blaming oneself for negative external events, guilt, and personalisation (Abramowitz et al., 2003; Berle \& Starcevic, 2005).

The investigation of TAF and its relation to a wide variety of disorders is important for understanding these disorders. If TAF is shown to be related to a range of disorders and variables, then it may be more helpful to consider it as a more general cognitive bias, rather than a specific component or symptom of OCD (Abramowitz et al., 2003). In the most comprehensive analysis to date, Marino, Lunt and Negy (2008) used Structural Equation Modelling (SEM) to examine the relationships between a number of variables associated with TAF, including responsibility, magical ideation, ethnicity, cognitive intrusions, obsessive-compulsive symptoms and religiosity. The participants used for this study were 714 undergraduate psychology students. The results from this investigation yielded many interesting findings. First, religiosity was found to be a significant predictor of TAF beliefs, partially mediated by an inflated sense of responsibility in that the more religious a person considers themselves to be, the higher their likelihood of engaging in TAF beliefs. It was also found that both TAF and OC symptoms were predictors of 
neutralising behaviour. The results showed that magical thinking was not a predictor for either TAF or OC symptoms and was not a significant factor in the model, as had been found in previous research (Marino et al., 2008). Unlike Smari and Holmsteinsson (2001), who investigated thought suppression as a mediating variable between responsibility beliefs and OC symptoms, Marino et al. (2008) used neutralisation as the mediator, which was found to be significant.

More recently, Altin and Gencoz (2011) used mediation analyses to investigate the relationships between TAF, thought suppression and OC symptoms, using a non-clinical, undergraduate sample. The results from their study indicate that the two components of TAF each followed different paths to OC symptomology. While TAF-moral was associated with inflated responsibility, TAF-likelihood was related to thought suppression, which aggravated OC symptoms. The authors suggest that believing that thoughts increase the likelihood of a negative event motivates one to suppress these thoughts, in turn leading to an activation of OC symptoms. These are in contrast with the findings of Rassin et al. (2000) discussed earlier who found TAF-likelihood to have a direct path to OCD, and TAF-moral to increase OCD symptoms through thought suppression.

\section{$2.2 \quad$ Study 1}

The goal of Study 1 was to examine the effects of both TAF and Inflated Responsibility on OC symptoms thus replicating the relationship previously demonstrated. Previous research in this area has focused solely on OCD; however the current study also examined the effect of these same factors on the non-OC psychopathologies of anxiety and depression. This will provide evidence of both constructs as general cognitive biases, as opposed to being specifically related to obsessive compulsive symptoms. Additionally, the current study examined the potential mediating role of thought suppression.

\subsubsection{Hypotheses}

It was hypothesised that (1) TAF and Inflated responsibility would be significantly correlated with OC symptoms, depression and anxiety; (2) that the correlation between TAF and Responsibility with anxiety and depression would remain significant after controlling for OC symptoms, (3) that Inflated Responsibility 
beliefs would be predictive of OC symptoms, depression and anxiety, with these relationships being mediated by thought suppression; and (4) that TAF would be predictive of OC symptoms, depression and anxiety, with these relationships being mediated by thought suppression. In terms of the separate components of TAF (moral and likelihood), previous research findings in this area are mixed and contradictory, so the current analyses in this area are exploratory.

\subsection{Method}

\subsubsection{Participants}

The participants for this study were 193 undergraduate Psychology students from Victoria University of Wellington, who participated in groups of up to 10. For their participation, the students received one half hour credit towards their compulsory research participation. The sample consisted of 125 females (64.8\%) and 67 males (34.7\%). The participants ages ranged from 16 to 39 with a mean age of 19.23 $(\mathrm{SD}=2.59)$. The sample consisted mainly of New Zealand European participants (73.6\%), 7.3\% were Asian, with the remaining participants being European (4.7\%), Maori (4.7\%), Pacific Nations (3.1\%), and other (5.2\%).

\subsubsection{Measures}

The Obsessive Compulsive Inventory - Revised (OCI-R; Foa et al., 2002) was used to assess the degree to which participants experience symptoms of OCD. Responses were measured on a Likert scale ranging from 0 (not at all) to 4 (extremely), with a scale score calculated by summing the scores of all 18 items (higher scores indicated higher OC symptomology). The items included statements such as "I frequently get nasty thoughts and have difficulty in getting rid of them", and "I repeatedly check gas and water taps and light switches after turning them off". Foa et al. (2002) reported an alpha of .90 for the scale. A copy of this scale is included in Appendix E.

The White Bear Suppression Inventory (WBSI; Wegner \& Zanakos, 1994) is a 15 item unbalanced measure which assesses the extent to which individuals suppress specific thoughts. The items are measured on a Likert scale ranging from 1 (strongly disagree) to 7 (strongly agree). The items include statements such as "Sometimes I stay busy just to keep thoughts from intruding on my mind", and "I often do things to 
distract myself from my thoughts". This scale aims to measure to what extent an individual tries to get rid of particular thoughts. Wegner and Zanakos (1994) reported reliabilities ranging from .87 to .89 . The WBSI scale score was created by averaging the scores from all 15 items. Higher scores indicate a stronger tendency to engage in suppression. This scale is included in Appendix G.

The Thought-Action Fusion Scale - Revised (TAF-R; Shafran et al., 1996) is a 19 item scale which assesses the degree to which participants equate their thoughts with actions. Twelve of the items refer to TAF Moral (e.g. "Thinking of cheating in a personal relationship is almost as immoral to me as actually cheating"); four for TAF likelihood-others (e.g. "If I think of a relative/friend losing their job, this increases the risk that they will lose their job"); and three for TAF likelihood-self (e.g. "If I think of myself falling ill, this increases the risk that I will fall ill”). Each item is a statement to which the participants rate the extent to which they agree or disagree using a five point Likert scale. An overall scale score is calculated by summing the scores of all the items, with higher scores indicating a stronger TAF bias. A copy of this scale is included in Appendix $\mathrm{H}$.

The Responsibility Attitude Scale (RAS; Salkovskis et al., 2000) is a 26 item questionnaire used to assess general beliefs about responsibility. Items include statements such as "I must protect others from harm," and "I am too sensitive to feeling responsible for things going wrong". Participants rated how much they agreed or disagreed with each statement on a seven-point Likert scale ranging from 'Totally agree' to 'Totally disagree', with a total overall score calculated by summing all of the items, and higher scores indicating higher levels of inflated responsibility. This scale is included in Appendix F.

The Self-Rating Depression Scale (Zung, 1965) measures depression using a 20 item scale with items such as "I feel down-hearted and blue." Half of the items are reverse-scored. Participants rate how much well each statement describes the way they have been feeling in the past several days on a Likert scale ranging from 1 (a little of the time) to 4 (most of the time). Higher overall scores indicate a higher level of depression. A copy of this scale is in Appendix I.

The Self-Rating Anxiety Scale (Zung, 1971) was used to measure the level of anxiety of participants on a 20 item scale. Items include statements such as "I feel 
more nervous and anxious than usual," and "I can feel my heart beating fast". Five of the items are reverse-coded. Participants are required to indicate on a 4-point Likert scale how each item best describes the way they have felt or behaved during the past several days. Higher overall scores indicate higher levels of anxiety. This scale is included in Appendix $\mathrm{J}$.

\subsubsection{Procedure}

The questionnaires were completed in group sessions of up to 10 participants. Before commencing, participants were each given an information sheet to read, a consent form to sign and were given time to ask any questions they may have. The experimenter also introduced the participants to the study and informed them of the sensitivity of some of the questions in the survey. This was accompanied by the assurance that if at any time a participant chose to withdraw from the study, they could do so without penalty.

After the participants had completed their surveys, which took approximately 30 minutes, they were debriefed by the experimenter and given a debriefing sheet to take with them. The Victoria University Human Ethics Committee gave approval for this study prior to its beginning. The information sheet, questionnaire and the debriefing sheet are included in the Appendix A.

\section{$2.4 \quad$ Results}

\subsubsection{Descriptive Analyses}

The means, standard deviations and reliability coefficients for all latent variables are shown in Table 2.1, and Pearson correlations shown in Table 2.2. Correlations while controlling for Obsessive Compulsive symptoms are shown in Table 2.3. As seen in Table 2.1, all scales showed good to excellent reliability. 
Table 2.1

Means and standard deviations of latent variables

\begin{tabular}{llll}
\hline & M & SD & $\alpha$ \\
\hline TAF-R & 20.59 & 12.59 & .91 \\
RAS & 72.30 & 25.61 & .93 \\
WBSI & 2.83 & .82 & .92 \\
SRDS & 40.59 & 10.08 & .87 \\
SRAS & 37.56 & 10.78 & .90 \\
OCI-R & 16.01 & 10.89 & .90 \\
\hline
\end{tabular}

Note: $N=193 ; \alpha$ : Cronbach's alpha.

TAF-R: Thought-Action Fusion Scale - Revised (Shafran et al., 1996); RAS:

Responsibility Attitude Scale (Salkovskis et al., 2000); WBSI: White Bear

Suppression Inventory (Wegner \& Zanakos, 1994); SRDS: Self-Rating Depression

Scale (Zung, 1965); SRAS: Self-Rating Anxiety Scale (Zung, 1971); OCI-R:

Obsessive Compulsive Inventory - Revised (Foa et al., 2002).

Table 2.2

Pearson correlations among latent variables with likelihood and moral TAF subscales

\begin{tabular}{|c|c|c|c|c|c|c|c|c|}
\hline & 1 & 2 & 3 & 4 & 5 & 6 & 7 & 8 \\
\hline (1) TAF & 1 & & & & & & & \\
\hline (2) Moral & $.91 * *$ & 1 & & & & & & \\
\hline (3) Likelihood & $.59 * *$ & $.19 *$ & 1 & & & & & \\
\hline (4)Responsibility & $.34 * *$ & $.28 * *$ & $.23 * *$ & 1 & & & & \\
\hline (5) WBSI & $.30 * *$ & $.15^{*}$ & $.39 * *$ & $.46^{* *}$ & 1 & & & \\
\hline (6) Depression & $.19 * *$ & .02 & $.38 * *$ & $.40 * *$ & $.57 * *$ & 1 & & \\
\hline (7) Anxiety & $.26 * *$ & .09 & $.41 * *$ & $.42 * *$ & $.60 * *$ & $.84 * *$ & 1 & \\
\hline (8) $\mathrm{OCI}$ & $.24 * *$ & .06 & $.39 * *$ & $.45^{* *}$ & $.61 * *$ & $.59 * *$ & $.62 * *$ & 1 \\
\hline
\end{tabular}

Note: $N=193 ;{ }^{* *} p<.01,{ }^{*} p<.05$. TAF-R: Thought-Action Fusion Scale - Revised (Shafran et al., 1996); RAS: Responsibility Attitude Scale (Salkovskis et al., 2000); WBSI: White Bear Suppression Inventory (Wegner \& Zanakos, 1994); SRDS: SelfRating Depression Scale (Zung, 1965); SRAS: Self-Rating Anxiety Scale (Zung, 1971); OCI-R: Obsessive Compulsive Inventory - Revised (Foa et al., 2002). 
Correlation analyses show that all overall scales are significantly related to each other. When looking at the TAF subscales, Likelihood is significantly related to all other variables, while Moral does not significantly correlate with depression, anxiety and OC symptoms. After controlling for OC symptoms (Table 2.3), the correlation between TAF and thought suppression, depression and anxiety all become non-significant. However inflated responsibility remains significantly correlated with thought suppression, depression and anxiety. The two subscales of TAF produce mixed results. These preliminary results indicate that overall TAF is more specific to OC symptoms, and related to other disorders and thought suppression through these. However inflated responsibility remains significantly related to thought suppression, anxiety and depression even when OC symptoms are controlled for, suggesting it may be a more general cognitive bias.

Table 2.3

Pearson correlations among latent variables controlling for Obsessive-Compulsive symptoms

\begin{tabular}{llllllll}
\hline & 1 & 2 & 3 & 4 & 5 & 6 & 7 \\
\hline (1) TAF & 1 & & & & & & \\
(2) Moral & $.91^{* *}$ & 1 & & & & & \\
(3) Likelihood & $.50^{* *}$ & .09 & 1 & & & & \\
(4)Responsibility & $.21^{*}$ & $.23^{*}$ & .02 & 1 & & & \\
(5) WBSI & .14 & .10 & .13 & $.24^{*}$ & 1 & & \\
(6) Depression & .05 & -.05 & $.21^{*}$ & $.24^{*}$ & $.31^{* *}$ & 1 & \\
(7) Anxiety & .12 & .02 & $.25^{* *}$ & $.22^{*}$ & $.37^{* *}$ & $.76^{* *}$ & 1
\end{tabular}

Note: $N=193 ; *^{*} p<.01,{ }^{*} \mathrm{p}<.05$ TAF-R: Thought-Action Fusion Scale - Revised (Shafran et al., 1996); RAS: Responsibility Attitude Scale (Salkovskis et al., 2000); WBSI: White Bear Suppression Inventory (Wegner \& Zanakos, 1994); SRDS: SelfRating Depression Scale (Zung, 1965); SRAS: Self-Rating Anxiety Scale (Zung, 1971).

Each of the constructs under investigation served as latent variables in the models used in this study. Obsessive-Compulsive Symptoms were indicated by the checking, hoarding, neutralising, obsessing, ordering, and washing subscales measured by the OCI. Thought-Action fusion was indicated by the moral, likelihoodself and likelihood-other subscales of the TAF-R. For all of the other latent variables, 
three parcels of items from each of the scales were used in the measurement model as indicators of the construct.

\subsubsection{Model Analyses}

Data analyses were performed using Analysis of Moment Structures (AMOS). Overall model fit was examined using the Chi-squared ratio (chi-square value divided by the degrees of freedom, which should be less than 4.0 or 5.0 (Jose, 2010)), the comparative fit index (CFI), the squared error of approximation (RMSEA), and Hoelter's Critical Number (CN). CFI values greater than .90 (Bentler, 1992) and RMSEA values less than .10 (Kline, 1998) indicated acceptable model fit. The last goodness-of-fit statistic is Hoelter's Critical Number $(\mathrm{CN})$. According to Hoelter (1983), a value that exceeds 200 is indicative of a model that represents the sample data.

\subsubsection{Measurement Models}

The initial measurement models allowed all latent constructs to correlate freely, with satisfactory $\mathrm{x}^{2} / \mathrm{df}, \mathrm{CFI}$, and RMSEA values shown in Table 2.4. The Outcome measurement model (Depression, Anxiety and OC symptoms) is shown in Figure 2.4, and the Predictor measurement model (Inflated Responsibility and Thought-Action Fusion) is shown in Figure 2.5. For variables that did not have subscales (Depression, Anxiety and Inflated Responsibility), parcels of items were created and used to indicate the variables. The parcelling of items refers to the creation of small bundles of individual scale items, which are then submitted to be analysed. 


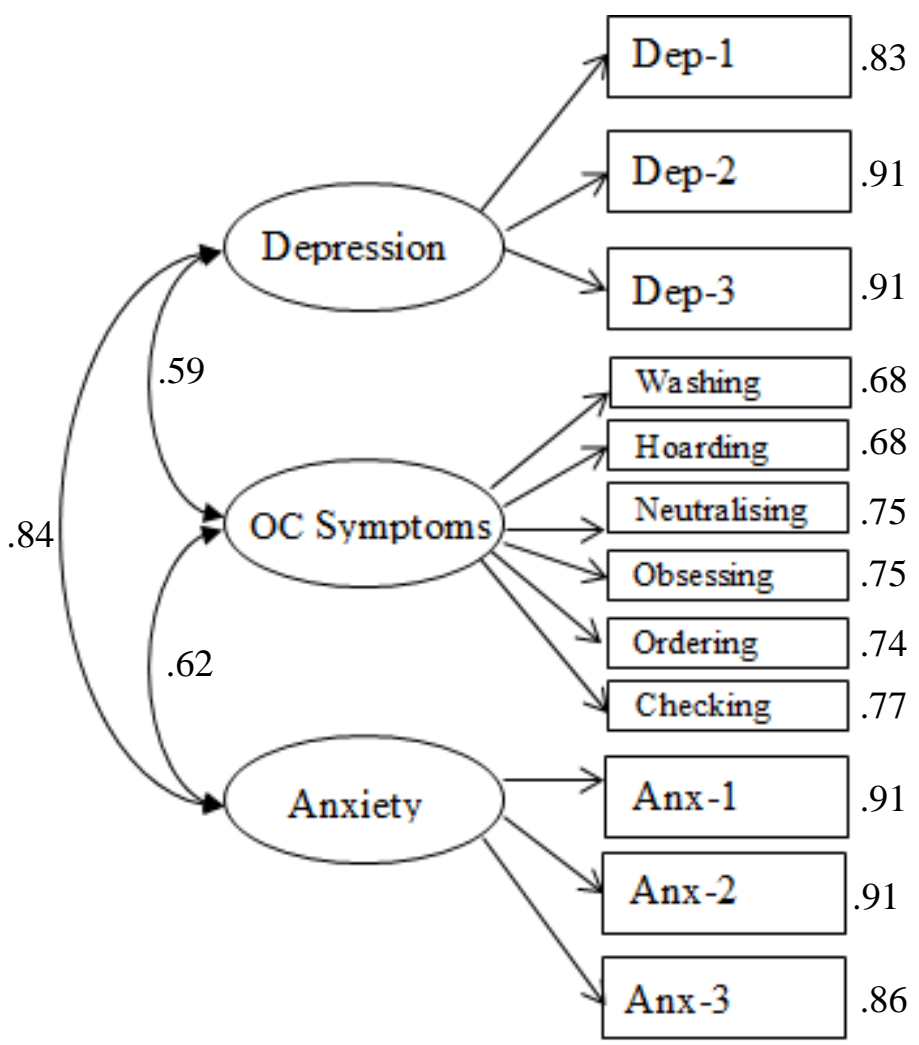

Figure 2.2 Outcome Measurement Model.

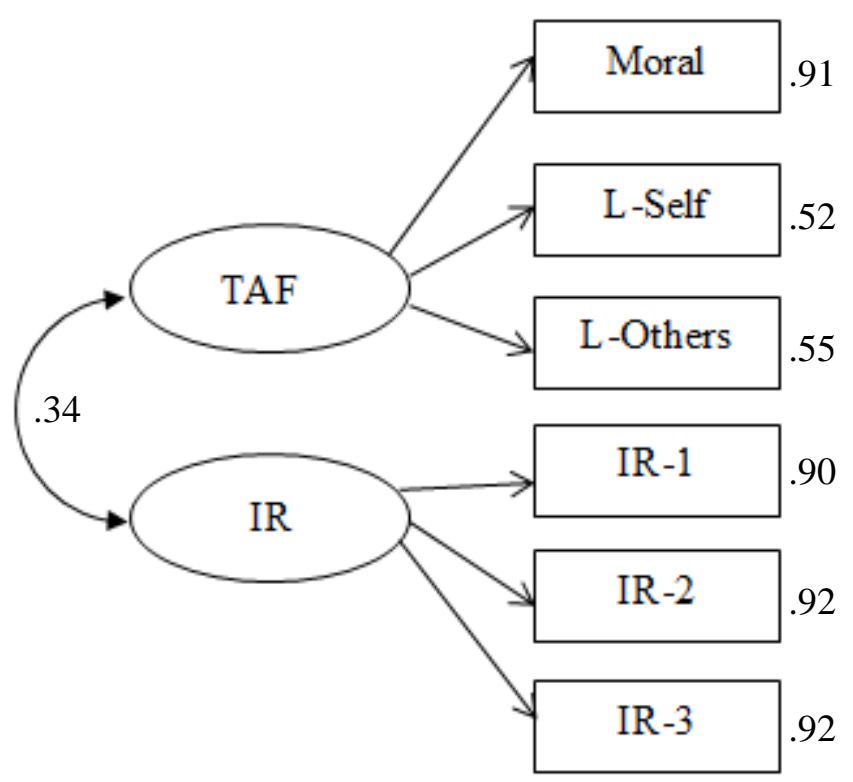

Figure 2.3 Predictor Measurement Model 
Table 2.4

Fit indices for Measurement Models

\begin{tabular}{lrccc}
\hline Model & $\mathrm{x}^{2}$ & $\mathrm{x}^{2} / \mathrm{df}$ & CFI & RMSEA \\
\hline Outcome Measurement model & 92.80 & $1.82^{\mathrm{a}}$ & $.97^{\mathrm{a}}$ & $.07^{\mathrm{a}}$ \\
Predictor Measurement model & 27.74 & $3.47^{\mathrm{a}}$ & $.96^{\mathrm{a}}$ & .10
\end{tabular}

Note: $N=193 .{ }^{a}$ Indicative of a good fit. Outcome measurement model: Depression, Anxiety, OC Symptoms. Predictor measurement model: Thought-Action Fusion and Inflated Responsibility.

\subsubsection{Structural Modelling}

\subsubsection{Inflated Responsibility}

For Inflated Responsibility, models were run with thought suppression as a full mediator (Figure 2.4) and a partial mediator (Figure 2.5). For the partial mediation model, the association between depression and OC symptoms was removed to avoid a just identified model (and get model fit indices). A nested model (Figure 2.6) excluding thought suppression was also run to check for a more economical model fit. The model fit indices for these models are shown in Table 2.5, and indicate that the partial mediation model best represents the sample data demonstrating the better fitting and more parsimonious model.

Table 2.5

Inflated Responsibility: Fit indices for covariance structure analyses

\begin{tabular}{lllllll}
\hline Model & $\mathrm{x}^{2}$ & $\mathrm{df}$ & $\mathrm{x}^{2} / \mathrm{df}$ & CFI & RMSEA & $\begin{array}{l}\text { CN } \\
(0.1)\end{array}$ \\
\hline Full Mediation Model & 12.29 & 3 & 4.10 & $.98^{\mathrm{a}}$ & .13 & 178 \\
Partial Mediation Model & 1.94 & 1 & $1.94^{\mathrm{a}}$ & $.99^{\mathrm{a}}$ & $.07^{\mathrm{a}}$ & $656^{\mathrm{a}}$ \\
Nested Model & 2.84 & 1 & $2.84^{\mathrm{a}}$ & $.99^{\mathrm{a}}$ & $.09^{\mathrm{a}}$ & $448^{\mathrm{a}}$ \\
\hline
\end{tabular}

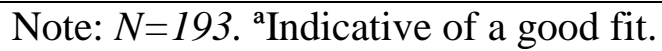




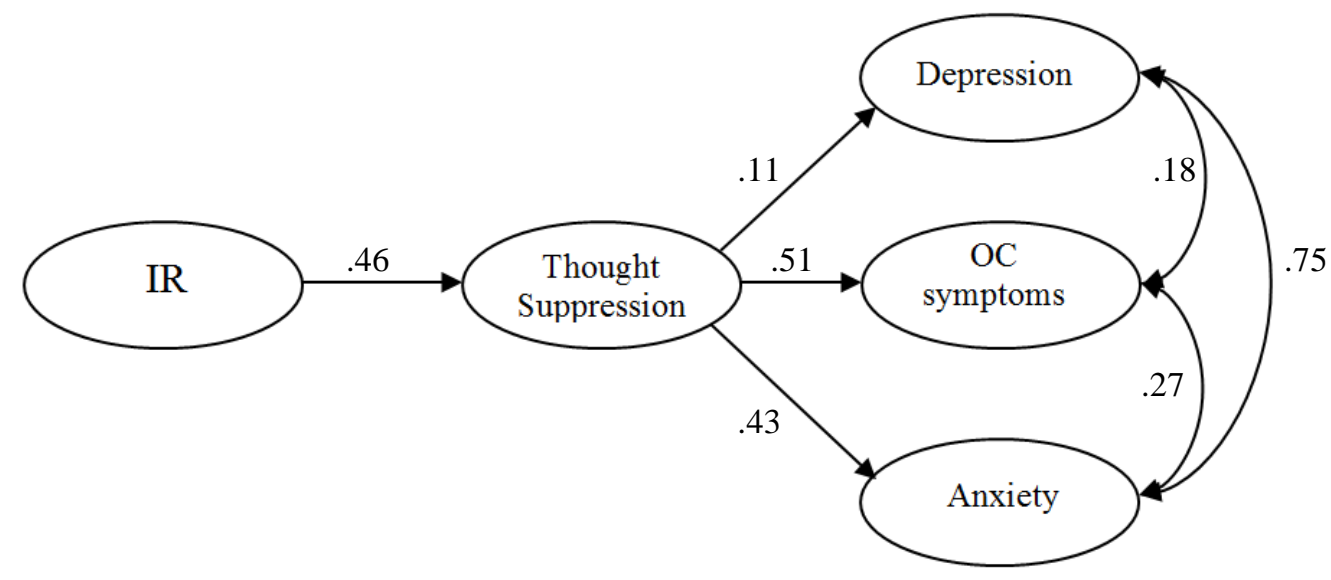

Figure 2.4 Inflated Responsibility (IR): Full mediation model with standardised regression weights

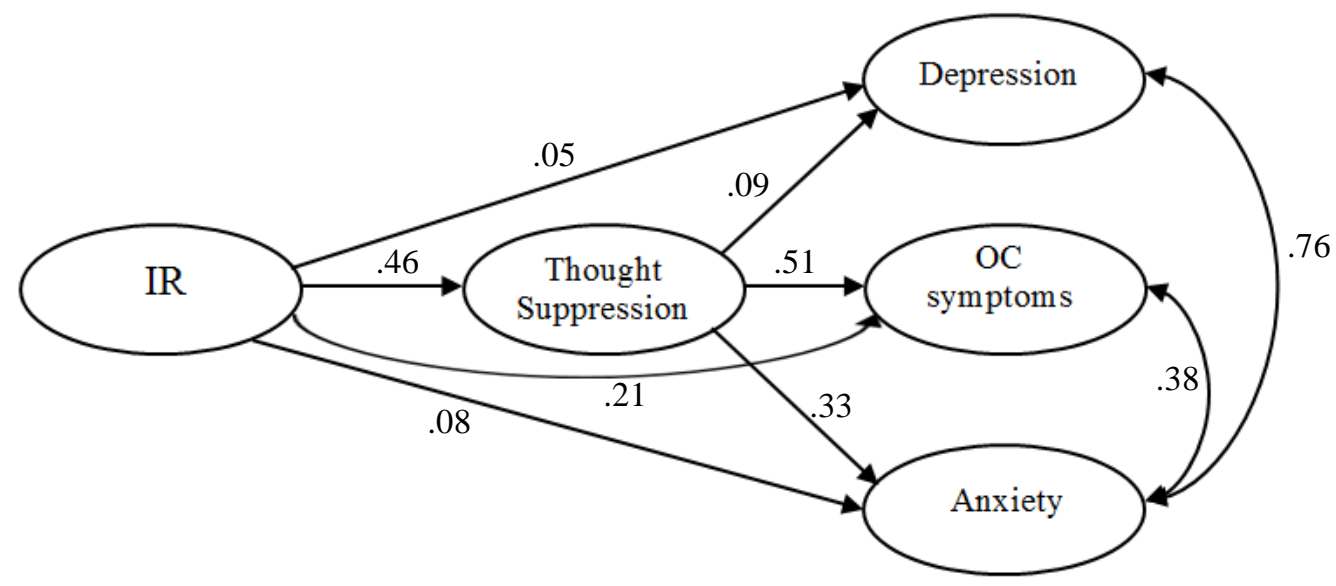

Figure 2.5 Inflated Responsibility (IR): Partial mediation model with standardised regression weights

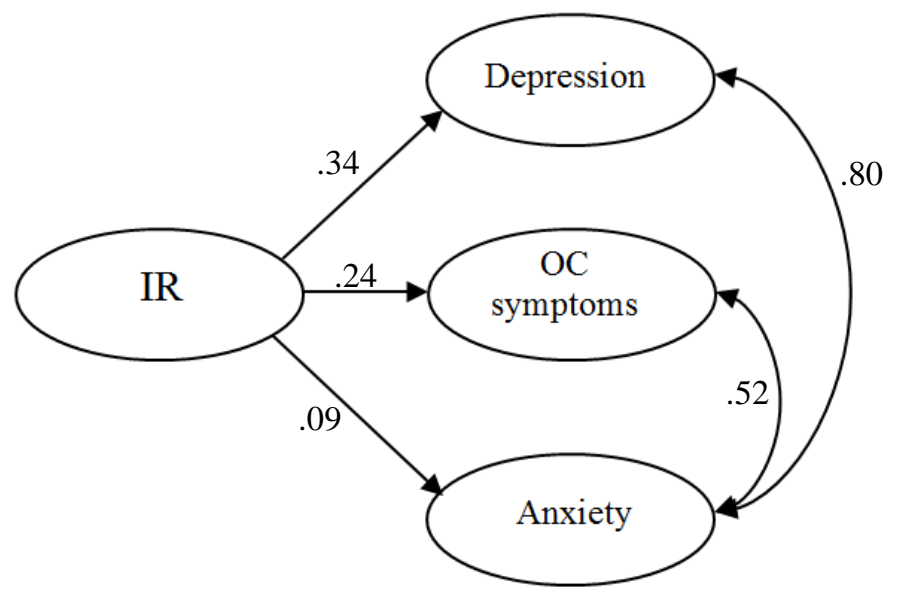

Figure 2.6 Inflated Responsibility (IR): Nested model with standardised regression weights 


\subsubsection{Thought-Action Fusion}

Similarly for Thought-Action Fusion, models were run with thought suppression as a full mediator (Figure 2.7) and a partial mediator (Figure 2.8). A nested model (Figure 2.9) excluding thought suppression was also run to check for a more economical model fit. Finally, a mixed model separating TAF into Moral and Likelihood subscales was then run (Figure 2.10). The model included here displays a full mediation for TAF-moral, and partial mediation for TAF-likelihood. This is based on past research and manipulation of several models to find the better fit. The model fit indices for all models are shown in Table 2.6, and indicate that initially the full mediation model appears to be the better fitting option. However, TAF is made up of two different components (moral and likelihood). When these are separated, the mixed model includes thought suppression as both a partial and full mediator.

Table 2.6

Thought-Action Fusion: Fit indices for covariance structure analyses

\begin{tabular}{lcccccc}
\hline Model & $\mathrm{x}^{2}$ & $\mathrm{df}$ & $\mathrm{x}^{2} / \mathrm{df}$ & $\mathrm{CFI}$ & RMSEA & $\begin{array}{l}\mathrm{CN} \\
(0.1)\end{array}$ \\
\hline Full Mediation Model & 2.55 & 3 & $.85^{\mathrm{a}}$ & $1.00^{\mathrm{a}}$ & $.00^{\mathrm{a}}$ & $853^{\mathrm{a}}$ \\
Partial Mediation Model & 3.57 & 2 & $1.79^{\mathrm{a}}$ & $1.00^{\mathrm{a}}$ & $.06^{\mathrm{a}}$ & $495^{\mathrm{a}}$ \\
Nested Model & 4.93 & 1 & 4.93 & $.99^{\mathrm{a}}$ & .14 & $259^{\mathrm{a}}$ \\
Mixed Model & 2.26 & 5 & $2.26^{\mathrm{a}}$ & $.99^{\mathrm{a}}$ & $.08^{\mathrm{a}}$ & $257^{\mathrm{a}}$
\end{tabular}

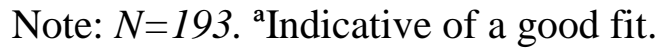




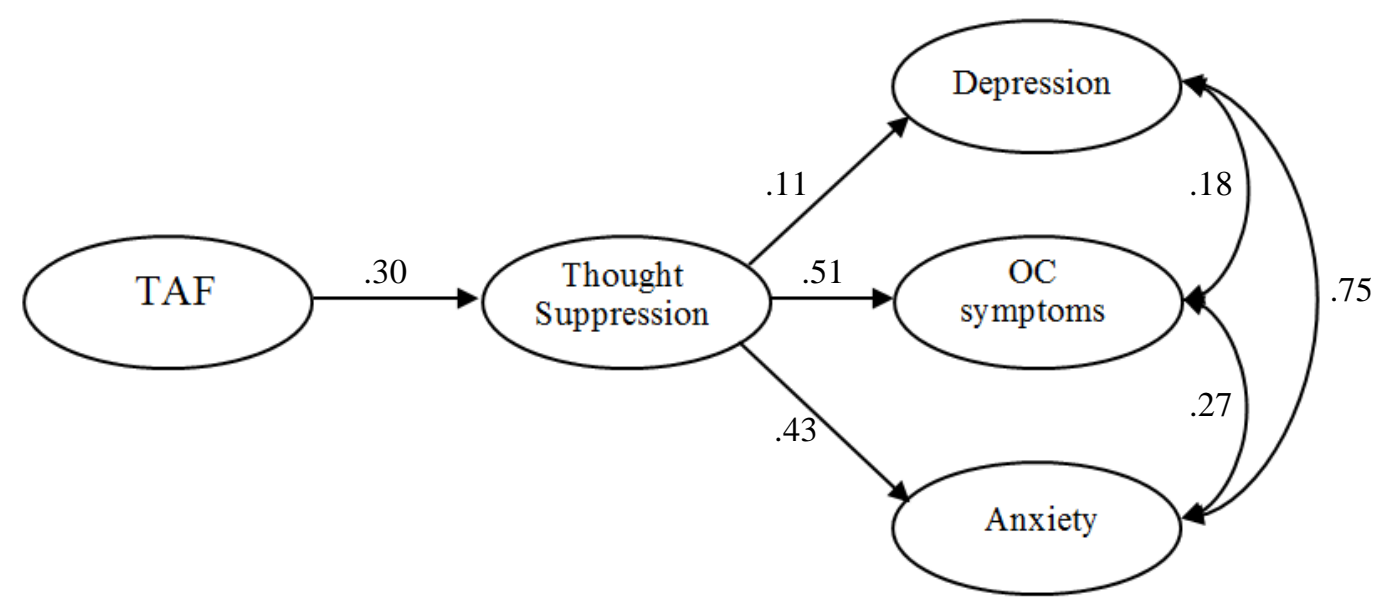

Figure 2.7 Thought-Action Fusion (TAF): Full mediation model with standardised regression weights

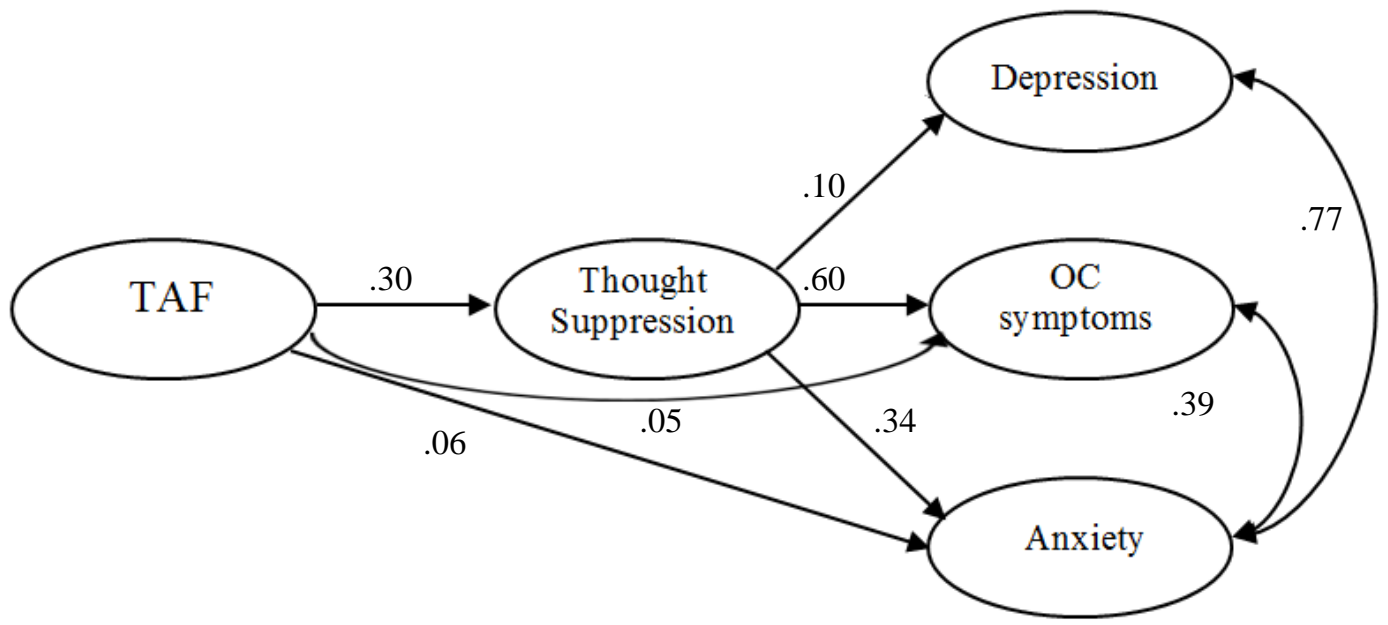

Figure 2.8 Thought-Action Fusion (TAF): Partial mediation model with standardised regression weights

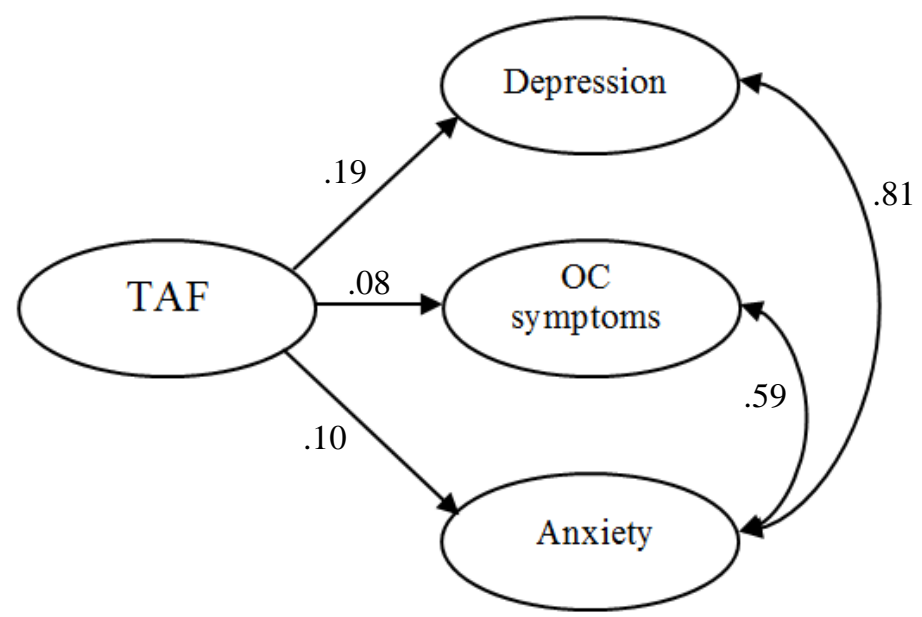

Figure 2.9 Thought-Action Fusion (TAF): Nested model with standardised regression weights 


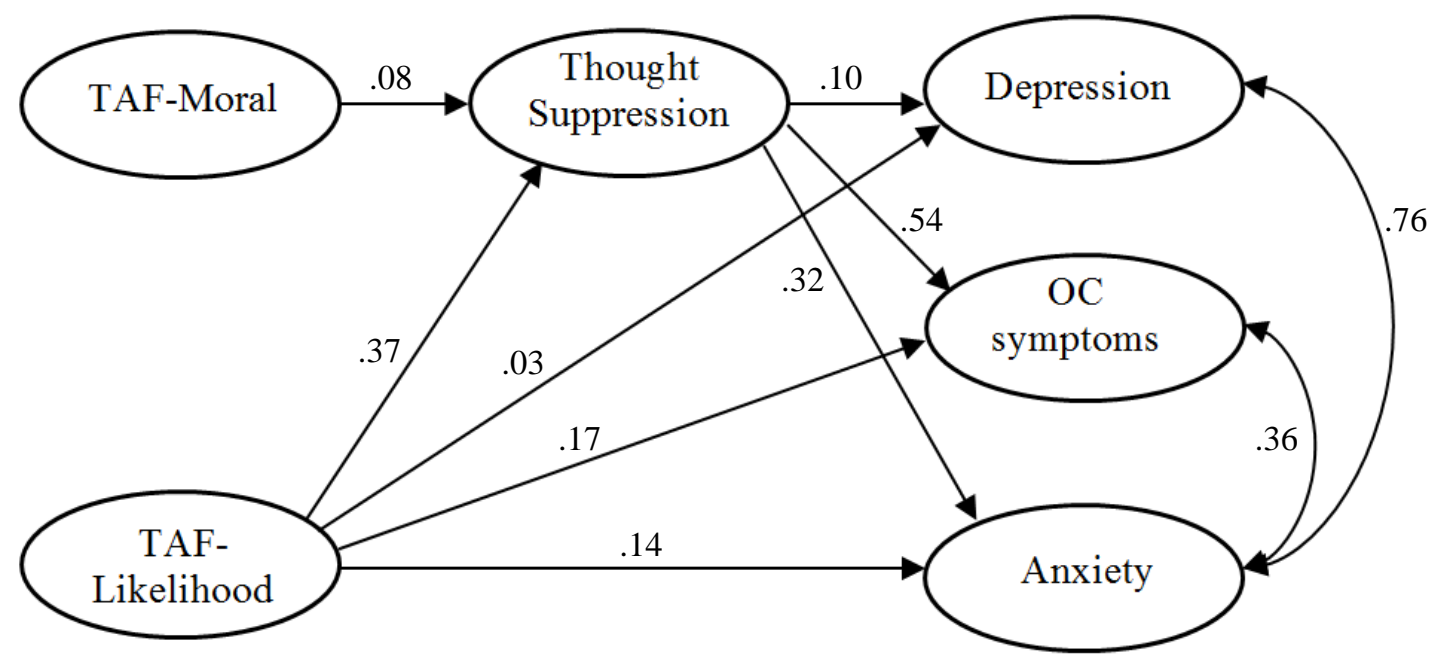

Figure 2.10 Thought-Action Fusion (TAF): Mixed model of moral and likelihood subscales with standardised regression weights

\subsection{Study 1 Discussion}

The aim of Study 1 was to investigate the relationships between inflated responsibility and TAF and psychopathology, namely OC symptoms, depression and anxiety. The overall results demonstrated interesting differences between TAF and inflated responsibility and their individual relationships with OC symptoms, anxiety and depression, as well as their relationship with thought suppression. Firstly, while allowing all variables to correlate freely with each other, both variables were significantly related to all three disorders. However, when controlling for OC symptoms, the relationship with inflated responsibility remained significant, while that for TAF became non-significant. This suggests that while inflated responsibility may be a more generalised, broad cognitive bias, TAF seems to be more specifically related to OC symptoms.

The structural modelling results demonstrated the role of thought suppression between responsibility beliefs (both inflated responsibility and overall TAF), and OC symptoms, depression and anxiety. For inflated responsibility, overall model fit was better when thought suppression partially mediated the relationship with OC symptoms, depression and anxiety. However, for thought-action fusion, a mixed model produced the best results, with TAF-likelihood being directly linked to OC symptoms, depression and anxiety, while this association with TAF-moral was mediated by thought suppression. This finding dovetails nicely with the findings of Rassin et al., (2000) who had similar results examining only OC symptoms. Rassin et al. argue that TAF-likelihood is more likely to lead directly to compulsions and 
neutralising techniques associated with OCD and anxiety in order to prevent the negative event. However TAF-moral beliefs tend to lead to thought suppression and rumination, which go on to induce symptoms of OCD, anxiety and depression.

According to Berle and Starcevic (2005), normal intrusive thoughts become pathological through the intermediary process of thought suppression. The current results provide some support for this idea in relation to inflated responsibility and TAF-moral. The underlying theory around how this may work is the paradoxical effects of thought suppression whereby negative, intrusive thoughts may be successfully suppressed short term, but will return later with increased frequency and intensity (Wegner et al., 1987). Therefore thought suppression is problematic in that it increases the negativity and salience around a thought, more than what would have occurred if no suppression attempts were made.

Thought suppression in the current study was measured using the White Bear Suppression Inventory (WBSI; Wegner \& Zanakos, 1994). One major criticism of this scale is that some of the items are attempting to measure the presence of cognitive intrusions, as opposed to attempts at suppressing these, consequently producing misleading results. Early analyses of the WBSI structure revealed one underlying factor: the tendency to use suppression as a mental control strategy, however subsequent studies have produced alternative results. One example of this is that of Blumberg (2000) who found three overall factors; the presence of unwanted, intrusive thoughts, thought suppression, and self-distraction (as an avoidance strategy). Blumberg describes that overall high scores on this scale may therefore identify people who are not able to successfully suppress, and experience rebounds. Similarly, Hoping and de Jong-Meyer (2003) found two factors, unwanted intrusive thoughts, and thought suppression, concluding that the major variable assessed by the WBSI is not thought suppression, but the presence of thought intrusions. This is problematic, as Rassin (2003) describes, it is important to distinguish between thought suppression and thought intrusions, as the frequency of intrusions may be the direct result of unsuccessful thought suppression.

Rachman and Shafran (1999) identified both inflated responsibility and guilt in the development and maintenance of depression. It is possible that rather than trying to suppress their negative, intrusive thoughts, individuals dwell and ruminate on them. Rumination and repetitive negative thinking has been repeatedly implicated in depression (Papageorgiou \& Siegle, 2003), and can also be characteristic of anxiety 
disorders (Nolen-Hoeksema, 2000). This process would bypass the attempts at suppression, as upon experiencing a negative intrusive thought, the individual would then focus on that thought, not attempting to suppress or stifle it.

Results revealed that TAF was not correlated with depression and anxiety when OC symptoms were controlled for, and that thought suppression played a mediating role between TAF-moral and psychopathology. This indicates that TAF is likely to be a specific feature of OCD, and works alongside thought suppression in developing and maintaining symptoms, an interaction supported by research and theory (e.g. Rassin, 2001; Rassin, Merckelbach, Muris \& Spaan, 1999; Rachman, 1998). Along these lines, the individual experiences a TAF thought, they attempt to suppress it thereby paradoxically increasing its frequency and intensity, leading to OC symptoms and the urge to neutralise (Rassin, 2001). This would suggest that not only having TAF-related intrusions is important, but also the desire to suppress this kind of thinking is what potentially leads to distress and OC symptoms. For someone to want to suppress these feelings, they must be interpreting them as significantly undesirable and damaging in some way. It is therefore important that treatments are in place which can teach the individual new skills for coping with and reinterpreting negative intrusive thoughts, rather than attempting to avoid them.

The repeated experience of having a negative intrusion, interpreting it using TAF concepts, failed attempts at suppression leading to increased symptoms, results in a vicious cycle which is hard for the individual to stop, and can be very resistant to treatment. Exacerbating this cycle are the negative effects of attempting to suppress negative thoughts, which can have detrimental effects on the individual's mood (Purdon \& Clark, 2001). Research has shown that it is more difficult to suppress negative thoughts while the mood is negative (e.g. Wenzlaff, Wegner \& Roper, 1988; Wenzlaff, Wegner \& Klein, 1991). Additionally, these unsuccessful attempts at suppressing negative thoughts can be experienced as intense failure, further fuelling the already negative mood (Purdon \& Clark, 2001).

\subsubsection{Clinical Implications}

That inflated responsibility was found to remain significantly related to depression and general anxiety after controlling for OC symptoms has important implications for the treatment of both disorders. Inflated responsibility has always been considered in relation to $\mathrm{OCD}$, so giving this some attention in terms of treating depression and other types of anxiety may be beneficial. Rachman and Shafran (1999) 
suggest that depression can be the result of inflated responsibility beliefs and guilt. Targeting these thought patterns through therapy, and educating people on how these beliefs may be maintaining their disorders may prove to be helpful. A more specific example of this is applying a cognitive therapy programme targeting inflated responsibility to depressed and anxious patients, hopefully leading to a decrease in perceived responsibility, and therefore symptoms (Ladouceur, Leger, Rheaume \& Dube, 1996). Promising research in this area has shown inflated responsibility in OCD to be unstable and amenable to change (Rassin et al., 2000); it is anticipated that this will generalise to anxiety and depressive disorders.

The finding that the association between both inflated responsibility and TAF with symptoms of psychopathology in terms of thought suppression has some implications for treatment. Those who display more general inflated responsibility may benefit from treatments focussed on how they interpret thoughts in terms of guilt, and strategies for cognitive framing as opposed to ruminating on negative thoughts. However, because thought suppression only acted as a partial mediator for inflated responsibility, and not at all for TAF-likelihood, therapies targeted at thought suppression may be beneficial but may leave the underlying bias somewhat intact. Rassin et al. (2000) suggested that responsibility beliefs are likely to be a cause of OCD, unlike thought suppression which plays an intermediary role (rather than causal, or a result of OC symptoms) and suggested that by targeting responsibility biases through therapeutic interventions, this would therefore decrease the desire to engage in thought suppression. For both inflated responsibility and TAF, it is assumed that targeting the underlying cognitive biases will be more effective, as concluded by Rachman (1997, p. 299) that "the most direct and satisfactory treatment of obsessions is to assist the patient in the modification of the putatively causal catastrophic misinterpretations of the significance of their intrusive thoughts. Bluntly, if these misinterpretations are 'corrected', the obsession should cease".

\subsubsection{Limitations}

Study 1 has some limitations. It is important to note that while Structural Equation Modelling can be particularly useful for examining relationships between different variables, it cannot be used to infer causality (Marino et al., 2008). Additionally, the sample consisted of healthy volunteers, therefore findings cannot be generalised to those clinically affected by TAF and inflated responsibility, as well as 
clinical OCD, depression and anxiety. Future research with clinical participants is therefore recommended.

\subsection{Chapter Summary}

The overall findings of Study 1 highlight key differences between inflated responsibility and TAF in relation to psychopathology. It was found that inflated responsibility is significantly correlated with OC symptoms, depression and anxiety. This remained after controlling for OC symptoms, suggesting it is more of an overall cognitive bias which has important implications for its treatment. In contrast, while TAF was initially significantly correlated with OC symptoms, depression and anxiety, this became non-significant when OC symptoms were controlled for. This suggests that TAF is more of a specific symptom of OCD. It was also found that the structural model of inflated responsibility being related to OC symptoms, depression and anxiety was more parsimonious when partially mediated by thought suppression. It may be that upon experiencing negative intrusions, individuals will not attempt to suppress the thought, but will ruminate and focus on them. Additionally, thought suppression played a mediating role between TAF-moral and OC symptoms, anxiety and depression, while the association between TAF-likelihood and psychopathological symptoms was direct. These results have interesting implications for clinical treatment in terms of targeting the underlying responsibility biases that may lead to the desire to suppress negative thoughts. 


\section{CHAPTER THREE}

\section{RESPONSIBILITY BELIEFS AND PERCEIVED CONTROL}

The results from Study 1 highlighted the role of TAF and inflated responsibility in OC symptoms, depression and anxiety. The aim of Studies 2A and $2 \mathrm{~B}$ is to expand on these results by focusing on how responsibility beliefs may interact with beliefs about personal control, to exacerbate and maintain these symptoms. Research in this area has previously been conducted with Turkish adolescents (Altin \& Karanci, 2008). Studies 2A and 2B extend and improve on this by utilising adult samples, and with Study $2 \mathrm{~B}$ using a clinical sample of individuals with diagnosed anxiety disorders. Study $2 \mathrm{~B}$ is also the first study to examine TAF alongside control beliefs and $\mathrm{OC}$ symptoms, as an alternative to inflated responsibility.

\subsection{Control Beliefs and Obsessive Compulsive Disorder}

There has been recent interest focusing on cognitive factors and their role in developing and maintaining problematic responsibility beliefs in relation to $\mathrm{OC}$ symptoms, particularly around control beliefs (Altin \& Karanci, 2008). Most past research on OC symptoms and control theories focused on the need and ability to control thoughts. This literature focuses on two main concepts: the sense of control, and desire for control. Based on this research, evidence suggests that people with OCD report a greater desire for control over their thoughts than normal and anxious individuals (Clark, Purdon \& Wang, 2003). In terms of sense of control, these scores tend to be related to higher scores of OC symptomatology (e.g. Zebb \& Moore, 2003). Additionally, individuals with OCD demonstrate a high desire to control their thoughts, inconsistent with their low sense of control over their thoughts. This discrepancy leads to distress, as well as neutralisation behaviours and thought suppression (Moulding \& Kyrios, 2006). An example of such research is that of Moulding and Kyrios (2007) who investigated desire for control and sense of control and their relationship to OC symptoms with an undergraduate population. After controlling for depression and anxiety, higher levels of desire for control and lower levels of sense of control were associated with higher levels of OC symptoms and related beliefs. 


\subsection{Locus of Control}

Few studies have considered more general control theories in relation to OC symptoms, such as Locus of Control (LOC). This refers to attributions an individual places on the responsibility for their successes and failures in life (Rotter, 1966, 1975; as cited in Bernard \& Krupat, 1994). People with an internal locus of control believe that they are in control of their lives, and they take responsibility for what happens to them. Alternatively, those with an external locus believe that events in their lives are controlled by other people or chance. Following on from this, individuals with an internal locus are considered to be at an advantage, as they believe they have control over their own lives, whereas externals believe they have little or no influence (Kennedy, Lynch \& Schwab, 1998). Arguably, those individuals who have an inflated sense of responsibility and an external locus of control, would experience anxiety symptoms. That is, the level of perceived responsibility is not matched by their sense of control over a situation and this mismatch is likely to be highly aversive.

A large body of research has examined the effects of locus of control on symptoms of depression. The finding of greater externality in terms of locus of

control being associated with greater levels of depression has been demonstrated to be quite reliable (e.g. Ganellen \& Blaney, 1984; Burger, 1984; Benassi, Sweeney \& Dufour, 1988). Other research has suggested that locus of control can act as a mediator between life stressors and overall mental health. For example, Anderson (1977 cited in Parkes, 1984) found that internals reported lower levels of overall distress and demonstrated particular types of coping strategies focused more on taskcentred rather than emotion-centred behaviours. Similar research on the mediating effect of control beliefs was conducted by Hofman (2005) with individuals suffering from social anxiety. These results demonstrated a partial mediating effect of perceived control between estimated social cost and anxiety. Specifically, participants diagnosed with social anxiety disorder believed that social situations were more anxiety provoking when they perceived that they had little control over them.

In a recent study by Altin and Karanci (2008), the relationship between locus of control, inflated responsibility and non-clinical obsessive compulsive symptoms in Turkish adolescents was investigated. The results showed a significant interaction such that higher OC symptomology was associated with the combination of inflated responsibility along with an external locus of control. More specifically, for those 
individuals with inflated responsibility beliefs, OC symptoms were significantly higher with an external locus of control, by itself, compared to an internal locus of control. The overall findings suggest that simply having an external locus of control may not be sufficient to produce OC symptoms, but the addition of an inflated sense of responsibility for events and preventing harm, this may have a triggering effect on OC symptoms. Additionally when overall responsibility beliefs are low, and an external locus of control is present, the person is less likely to show OC symptoms. This research was limited in that it involved a very young non-clinical participant group (age range 16-20 years), and all measures were completed in Turkish, such that generalising the findings to those who have been diagnosed with OCD would be speculative at best.

\subsection{Study $2 A$}

Study 1 examined how inflated responsibility and TAF are related to psychopathology (namely OC symptoms, anxiety and depression), and the influence of thought suppression. Thought suppression can be considered a reaction to, or consequence of, inflated responsibility. It is something individuals do in response to unwanted responsibility beliefs. By targeting the underlying beliefs about responsibility, this should therefore decrease thought suppression attempts. It may be helpful to look more closely at responsibility beliefs and how individuals interpret them and their level of control. For example, if someone believes that they are responsible for preventing harm to themselves or others, their perception of how much control they have over the situation may be related to the level of anxiety they experience. The aim of Study 2A was to replicate the research of Altin and Karanci (2008), by investigating the effect of having an external locus of control on the relationship between inflated responsibility and OC symptoms, however with an adult, non-clinical participant group. If individuals believe that they are responsible for events in theirs and others' lives, and they also have an external locus of control, they would experience higher levels of OC symptoms.

\subsubsection{Hypotheses}

Based on previous research, it was predicted that: (1) both an inflated responsibility and external locus of control would significantly predict higher levels of OC symptoms, (2) that the interaction of the two variables would predict OC symptoms over and above that of each alone, (3) that for those individuals with a 
higher inflated sense of responsibility, OC symptom severity would be higher in those with higher levels of external locus of control.

\subsection{Method}

\subsubsection{Participants}

The participants for this study were 140 individuals who completed an online survey. Participants were recruited using a snowballing effect through the social networking site Facebook. The sample consisted of 78 females (56\%) and 24 males (17\%); 38 did not specify gender (27\%). The participants ages ranged from 16 to 66 with a mean age of $28.23(\mathrm{SD}=10.69)$.

\subsubsection{Measures}

The Obsessive Compulsive Inventory - Revised (OCI-R; Foa, et al., 2002), and the Responsibility Attitude Scale (RAS; Salkovskis et al., 2000) were used in this study and are described in detail in Study 1 (Chapter 2).

Additionally, Levenson's (1973) Multidimensional Locus of Control Inventory was used to measure internal and external locus of control. This is a 24 item scale, to which participants rated their agreement of a statement on a 6 point scale ranging from 'Agree Strongly' to 'Disagree Strongly'. The scale measured Internal Locus and External Locus. External was made up of the subscales Powerful Others and Chance. Statements include items such as "My life is chiefly controlled by powerful others" (external), and "I can pretty much determine what will happen in my life" (internal). Participants are able to obtain high or low scores on all three dimensions, with higher scores indicating a stronger endorsement for that attitude. For the purposes of this study, the Powerful Others and Chance subscales were combined to provide a measure of overall Externality. Only this combined External score was used in the current research. A copy of this scale is included in Appendix L.

\subsubsection{Procedure}

The questionnaire data were collected via an anonymous online survey using Survey Monkey. The initial sample of participants was invited to complete the survey by the principle investigator via connections on the Facebook social networking site. Subsequent participants were invited by those who had already completed the survey, or were those who randomly came across the site, using a snowballing effect. The survey itself took approximately 20 minutes to complete. The Victoria University Human Ethics Committee gave approval for this study prior to its beginning. The information sheet, questionnaire and the debriefing sheet are included in Appendix B. 


\subsection{Results}

\subsubsection{Descriptive Statistics}

The means, standard deviations and reliability coefficients for all variables are shown in Table 3.1. All scales showed good to excellent reliability.

Table 3.1

Means, standard deviations and Cronbach's alpha.

\begin{tabular}{llcc}
\hline & M & SD & $\alpha$ \\
\hline OCI & 36.91 & 13.59 & .92 \\
RAS & 69.38 & 33.86 & .91 \\
LOC - External & 54.18 & 11.38 & .82
\end{tabular}

Note: OCI-R: Obsessive Compulsive Inventory - Revised (Foa et al., 2002); RAS: Responsibility Attitude Scale (Salkovskis et al., 2000); LOC: Levenson's Multidimensional Locus of Control Inventory (Levenson, 1973).

Pearson correlations are presented in Table 3.2 OC symptoms, inflated responsibility and external locus of control all significantly correlated with each other.

Table 3.2

Pearson correlations

123

(1) OCI 1

(2) RAS $\quad .322 * * \quad 1$

(3) LOC - External $\quad .373 * * \quad .476 * * \quad 1$

Note: $* * p<.01$. OCI-R: Obsessive Compulsive Inventory - Revised (Foa et al., 2002); RAS: Responsibility Attitude Scale (Salkovskis et al., 2000); LOC:

Levenson's Multidimensional Locus of Control Inventory (Levenson, 1973).

\subsubsection{Multiple Regression}

Multiple regression analyses were performed to examine whether the interaction of inflated responsibility with an external locus of control would predict the presence of OC symptoms over and above the main effects of each individual variable. Table 3.3 shows the summary statistics for the hierarchical regression analysis with the total OC symptoms as the dependent variable. Inflated responsibility was entered into the equation as the first step and explained $14 \%$ of the variance $(t[139]=4.72, p<.001)$. External locus of control was entered in the second step 
explaining $19 \%$ of the variance, with both inflated responsibility $(t[138]=2.97$, $p<.001)$ and external locus of control $(t[138]=2.87, p<.001)$ being significant predictors. On the final step, the interaction term for inflated responsibility and external locus of control improved the overall variance to $25 \%$, and was a significant predictor of total OC symptoms $(t[137]=3.50, p<.001)$.

Table 3.3

The results of the multiple regression analyses

\begin{tabular}{|c|c|c|c|}
\hline & B & SE B & $\beta$ \\
\hline \multicolumn{4}{|l|}{ Step 1} \\
\hline Constant & 47.74 & 2.50 & \\
\hline Inflated Responsibility & .15 & .03 & $.37 *$ \\
\hline \multicolumn{4}{|l|}{ Step 2} \\
\hline Constant & 28.41 & 7.16 & \\
\hline Inflated Responsibility & .10 & .04 & $.26^{*}$ \\
\hline External Locus of Control & .30 & .16 & $.25^{*}$ \\
\hline \multicolumn{4}{|l|}{ Step 3} \\
\hline Constant & -1.38 & 10.94 & \\
\hline Interaction & .01 & .00 & $.92 *$ \\
\hline
\end{tabular}

Note: $R^{2}=.14$ for Step $1, \Delta R^{2}=.05(p<.001)$ for Step $2, \Delta R^{2}=.07(p<.001)$ for Step 3; $*=p<.001$. Dependent variable: OC symptoms

\subsubsection{Moderation Analyses}

The moderation analyses were performed using Modgraph (Jose, 2008), which is based on the guidelines by Aiken and West (1991). Before multiple regressions were performed, the two independent variables (External Locus of Control and Inflated Responsibility) were centred to avoid multicollinearity. Simple regression lines for moderated variables were plotted for significant interaction effects. As can be seen in Figure 3.1, inflated responsibility moderates the relationship from external Locus of Control to OC symptoms. That is, participants with high inflated responsibility showed more OC symptoms when paired with higher levels of Locus of Control externality. In fact, at every level of inflated responsibility, OC symptom severity was higher in those with higher levels of external Locus of Control.

The slopes of the three regression lines were tested to see if they significantly differed from zero. This revealed that the simple slopes for high inflated responsibility 
$(\beta=1.63, t[131]=3.91, p<.001)$, medium inflated responsibility $(\beta=1.36, t[131]=4.23$, $p<.001)$, and low inflated responsibility $(\beta=1.09, t[131]=5.54, p<.001)$ were all significant.

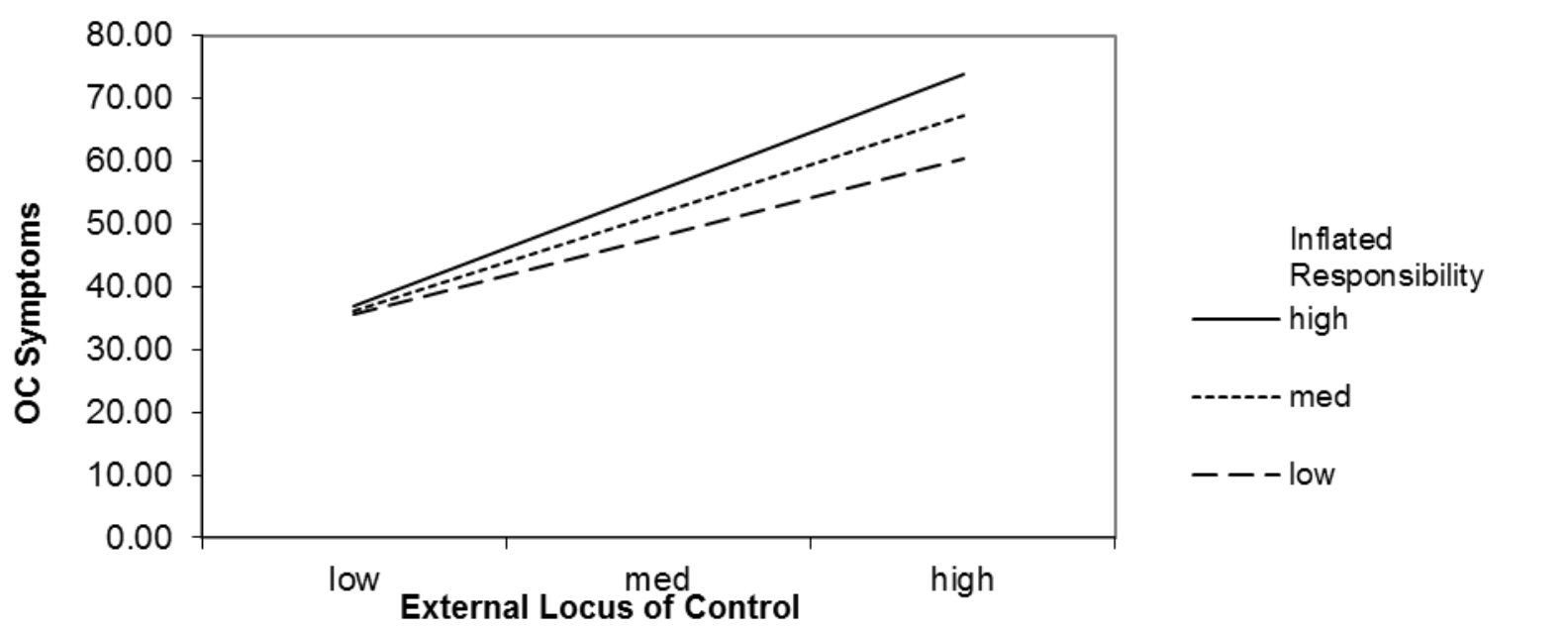

Figure 3.1 Interaction between Inflated Responsibility and external Locus of Control in the prediction of Obsessive Compulsive Symptoms.

\subsection{Study 2A Discussion}

The goal of Study 2A was to investigate the influence of external locus of control on the relationship between inflated responsibility and OC symptoms. The results showed that the interaction of inflated responsibility with an external locus of control significantly predicted OC symptoms more than each variable independently. Moderation results showed that having a higher sense of responsibility predicted higher OC symptom severity in those that also had an external locus of control. It is interesting to note that for all three levels of inflated responsibility, if an external locus of control was present, OC symptom severity was significantly higher than those who showed low levels of externality. This suggests that it is not simply the presence of inflated responsibility that predicts anxiety, but also how those beliefs are interpreted in regards to control. Evidently both variables are important and work together to produce feelings and symptoms of anxiety.

The current findings seem to be at odds with Altin and Karanci's (2008) findings in regards to inflated responsibility. Their results showed that when someone has an external locus of control, they also were only high on OC symptom severity if they also had high inflated responsibility. If the person had low responsibility beliefs, 
they were at lower risk of OC symptomatology even if they had an external locus of control. In the current study, however, all levels of inflated responsibility (low, medium, high) were related to higher OC symptomatology in the presence of external locus of control. Although all slopes were significant, results did show that, overall, higher levels of responsibility were related to OC symptom severity.

\subsection{Study 2B}

The aim of Study 2B was to replicate Study 2A with an adult clinical population. Results from Study 1 showed that TAF may be more specifically related to OC symptoms than inflated responsibility. While Study $2 \mathrm{~A}$ focussed solely on the interaction between external locus of control and inflated responsibility, in Study 2B a measure of thought-action fusion was included to see whether similar results would be found as an alternative type of responsibility belief. In the same way that inflated responsibility works alongside externality to exacerbate OC symptoms, the same is expected for TAF beliefs. This will be the first study to directly investigate thoughtaction fusion alongside locus of control and OC symptoms, and will provide information on how similar the two types of responsibility beliefs are, and to pinpoint similarities and differences in psychopathology.

\subsubsection{Hypotheses}

It was predicted that similar results would be found with the clinical sample as were found with Study 2A in terms of the interaction between inflated responsibility and externality predicting OC symptoms. That is, (1) that both an inflated responsibility and external locus of control would significantly predict higher levels of OC symptoms, (2) that the interaction of the two variables would predict OC symptoms over and above that of each alone, (3) that for those individuals with a higher inflated sense of responsibility, OC symptom severity would be higher in those with higher levels of external locus of control. Additionally, it was predicted that (4) the interaction between external locus of control and higher scores of thought-action fusion would lead to higher OC symptoms, similar to inflated responsibility.

\subsection{Method}

\subsubsection{Participants}

The participants for this study were 67 individuals who were seeking treatment for anxiety disorders. Participants completed an online survey, taking approximately 15-20 minutes. Of the participants, 28.4\% were diagnosed with OCD, $26.9 \%$ with Generalised Anxiety Disorder, $28.4 \%$ with Social Anxiety, 3\% with Panic 
Disorder, $1.5 \%$ with Agoraphobia, $7.5 \%$ with some other anxiety disorder, and $4.3 \%$ did not say. Males made up $43.1 \%$, and females made up $56.9 \%$ of the group. The mean age was $40.69(\mathrm{SD}=12.7)$ years, ranging from 18 to 67 .

\subsubsection{Measures}

The measures for Study 2B were identical to Study 2A with the addition of the TAF Scale. The Thought-Action Fusion Scale - Revised (TAF-R; Shafran et al., 1996) as described in Study 1 (Chapter 2).

\subsubsection{Procedure}

Individuals were invited to participate in the study via an email sent to clients, as well as in a newsletter of the Anxiety Clinic in Canterbury. The questionnaire data were collected via an anonymous online survey using Qualtrics software (Qualtrics, 2013). The Central Region Ethics Committee of the New Zealand Ministry of Health gave approval for this study prior to its beginning. The information, consent and debriefing information for this study is included in Appendix C.

\subsection{Results}

\subsubsection{Descriptive Statistics}

The means, standard deviations and reliability coefficients for all variables are shown in Table 3.4. All scales showed excellent reliability.

Table 3.4

Means, standard deviations and Cronbach's alpha.

\begin{tabular}{llll}
\hline & M & SD & $\alpha$ \\
\hline OCI & 66.63 & 18.57 & .91 \\
RAS & 139.78 & 32.55 & .97 \\
LOC - External & 66.53 & 14.15 & .91 \\
TAF - Total & 64.74 & 19.01 & .95 \\
TAF - Moral & 39.44 & 12.77 & .94 \\
TAF - Likelihood & 25.10 & 9.28 & .96
\end{tabular}

Note: OCI-R: Obsessive Compulsive Inventory - Revised (Foa et al., 2002); RAS:

Responsibility Attitude Scale (Salkovskis et al., 2000); LOC: Levenson's Multidimensional Locus of Control Inventory (Levenson, 1973); TAF: ThoughtAction Fusion Scale Revised (Shafran et al., 1996). 
Pearson correlations are presented in Table 3.5. All variables significantly correlated with each other, except for TAF-moral, which was only associated with overall TAF scores and TAF-likelihood.

Table 3.5

Pearson correlations

$\begin{array}{lllll}1 & 2 & 3 & 4 & 5\end{array}$

(1) OCI 1

(2) RAS $\quad .469 * * \quad 1$

(3) LOC - External $\quad .542 * * \quad .375^{* *} \quad 1$

(4) TAF - Total $\quad .317 * \quad .424 * \quad .321 * \quad 1$

$\begin{array}{llllll}\text { (5) TAF - Moral } & .193 & .293 & .153 & .910 * * & 1\end{array}$

(6) TAF - Likelihood $\quad .383 * * \quad .534 * * \quad .436 * * \quad .813 * * \quad .497 * *$

Note: $* * p<.01, * p<.05$. OCI-R: Obsessive Compulsive Inventory - Revised (Foa et al., 2002); RAS: Responsibility Attitude Scale (Salkovskis et al., 2000); LOC:

Levenson's Multidimensional Locus of Control Inventory (Levenson, 1973); TAF: Thought-Action Fusion Scale Revised (Shafran et al., 1996).

\subsubsection{Multiple Regression}

\subsubsection{Inflated Responsibility}

Multiple regression analyses were performed to examine whether the interaction of inflated responsibility and external locus of control would predict OC symptoms over and above the effects of each individual variable. Table 3.6 shows the summary statistics for the hierarchical regression analyses with the total OC symptoms as the dependent variable. Inflated responsibility was entered into the equation as the first step, and explained $16 \%$ of the variance $(t[66]=3.05, p<.001)$. External locus of control was entered in the second step explaining $29 \%$ of the variance with both inflated responsibility $(t[65]=2.56, p<.05)$ and external locus of control $(t[65]=2.92, p<.05)$ being significant predictors. On the final step, the interaction term for inflated responsibility and external locus of control did not improve the overall variance accounted for and was not a significant predictor of total OC symptoms $(t[64]=-.88, p=.38)$. 
Table 3.6

The results of the multiple regression analyses: Inflated Responsibility

B $\quad$ SE B $\quad \beta$

\begin{tabular}{|c|c|c|c|}
\hline \multicolumn{4}{|l|}{ Step 1} \\
\hline Constant & 66.42 & 2.39 & \\
\hline Inflated Responsibility & .26 & .08 & $.40 *$ \\
\hline \multicolumn{4}{|l|}{ Step 2} \\
\hline Constant & 66.21 & 2.22 & \\
\hline Inflated Responsibility & .21 & .08 & $.32 *$ \\
\hline External Locus of Control & .51 & .17 & $.37 *$ \\
\hline \multicolumn{4}{|l|}{ Step 3} \\
\hline Constant & 66.64 & 2.28 & \\
\hline Interaction & -.01 & .01 & -.17 \\
\hline
\end{tabular}

Note: $R^{2}=.16$ for Step 1, $\Delta R^{2}=.13(p<.001)$ for Step $2, \Delta R^{2}=.01(p=.38)$ for Step 3; $*=p<.001$. Dependent variable: OC symptoms

\subsubsection{Thought-Action Fusion}

Multiple regression analyses were also performed to examine whether the interaction of thought-action fusion and an external locus of control would predict overall OC symptoms. Table 3.7 shows the summary statistics for the hierarchical regression analysis with OC symptoms as the dependent variable. Thought-action fusion was entered as the first step in the equation and explained $10 \%$ of the variance $(t[66]=2.43, p<.05)$. External locus of control was entered in the second step explaining $32 \%$ of the variance. However, only locus of control was a significant predictor $(t[65]=4.01, p<.001)$. On the final step, the interaction term for thoughtaction fusion and external locus of control did not improve the overall variance accounted for, and was not a significant predictor of overall OC symptoms. 
Table 3.7

The results of the multiple regression analyses: Thought-Action Fusion

B $\quad$ SE B $\quad \beta$

\begin{tabular}{llll}
\hline Step 1 & & & \\
$\quad$ Constant & 65.88 & 2.40 & \\
$\quad$ Thought-Action Fusion & .32 & .13 & $.32 *$ \\
& & & \\
\hline
\end{tabular}

\begin{tabular}{llll}
\hline Step 2 & 66.50 & 2.11 & \\
Constant & .16 & .12 & .16 \\
$\quad$ Thought-Action Fusion & .64 & .16 & $.49^{*}$ \\
$\quad$ External Locus of Control & .64 & & \\
\hline Step 3 & 67.53 & 2.18 & \\
$\quad$ Constant & -.01 & .01 & -.19 \\
$\quad$ Interaction &
\end{tabular}

Note: $R^{2}=.10$ for Step $1, \Delta R^{2}=.05(p<.001)$ for Step $2, \Delta R^{2}=.07(p<.001)$ for Step 3; $*=p<.05$. Dependent variable: OC symptoms

The results from the regression analyses with thought-action fusion reveal that after external locus of control is entered into the model, TAF no longer predicts OC symptoms. This suggests that a mediational model may apply. In order for this, all three variables (the mediator, the independent variable, and the dependent variable) must be significantly correlated (Baron \& Kenny, 1986). The results of the correlational analyses demonstrate that external locus of control (the mediator), thought-action fusion (the independent variable), and OC symptoms (the dependent variable) were all interrelated, satisfying this condition. The association between thought-action fusion and OC symptoms must be reduced or non-significant with external locus of control in the model. The mediation analysis was performed using Medgraph (Jose, 2003). These results showed a full mediational model. A Sobel test was conducted in order to test for the indirect effect. A value of $z=2.13$ was found $(p<.05$; see Figure 3.2). 


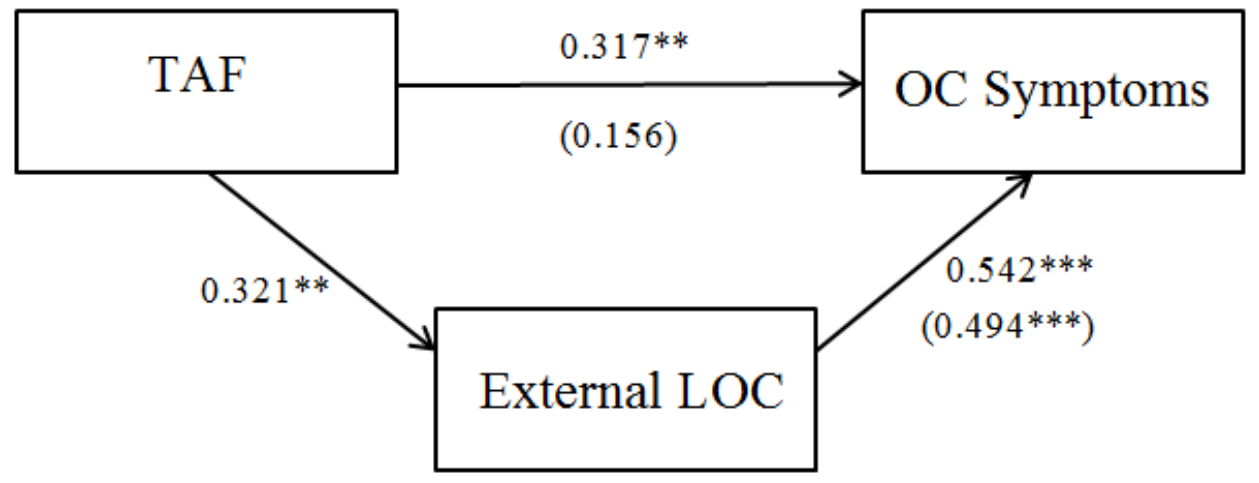

Figure 3.2 A mediational model, with External locus of control mediating between Thought-Action Fusion scores and OC symptoms; ${ }^{* *}=p<.05, * * *=p<.001$.

\subsection{TAF-likelihood and TAF-moral}

Multiple regression analyses were run for the two components of TAF-moral (Table 3.9) and likelihood (Table 3.8), with OC symptoms as the dependent variable. When TAF-likelihood was entered as the first step of the equation, it accounted for $15 \%$ of the variance $(t[66]=3.13, p<.05)$. When external locus of control was entered in the second step, it accounted for $30 \%$ of the variance, and was the only significant predictor $(t[65]=1.52, p<.001)$. On the final step, the interaction term for TAFlikelihood and external locus of control did not improve the overall variance accounted for, and was not a significant predictor of overall OC symptoms.

Table 3.8

The results of the multiple regression analyses: TAF-likelihood

\begin{tabular}{llll}
\hline & B & SE B & $\beta$ \\
\hline Step 1 & & & \\
Constant & 45.95 & 6.98 & \\
TAF-likelihood & .81 & .26 & $.38^{*}$ \\
& & & \\
\hline Step 2 & & & \\
Constant & 16.44 & 9.98 & .13 \\
TAF-likelihood & .39 & .26 & $.46^{* *}$ \\
External Locus of Control & .61 & .16 & \\
\hline Step 3 & & & \\
Constant & 18.86 & 10.03 & -.14 \\
Interaction & -.02 & .02 &
\end{tabular}

Note: $R^{2}=.15$ for Step $1, \Delta R^{2}=.18(p<.001)$ for Step $2, \Delta R^{2}=.02(p=.16)$ for Step 3; $*=p<.05, * *=p<.001$. Dependent variable: OC symptoms 
The mediation analysis was performed using Medgraph (Jose, 2003). These results showed a full mediational model. A Sobel test was conducted in order to test for the indirect effect. A value of $z=2.65$ was found ( $p<.05$; see Figure 3.3).

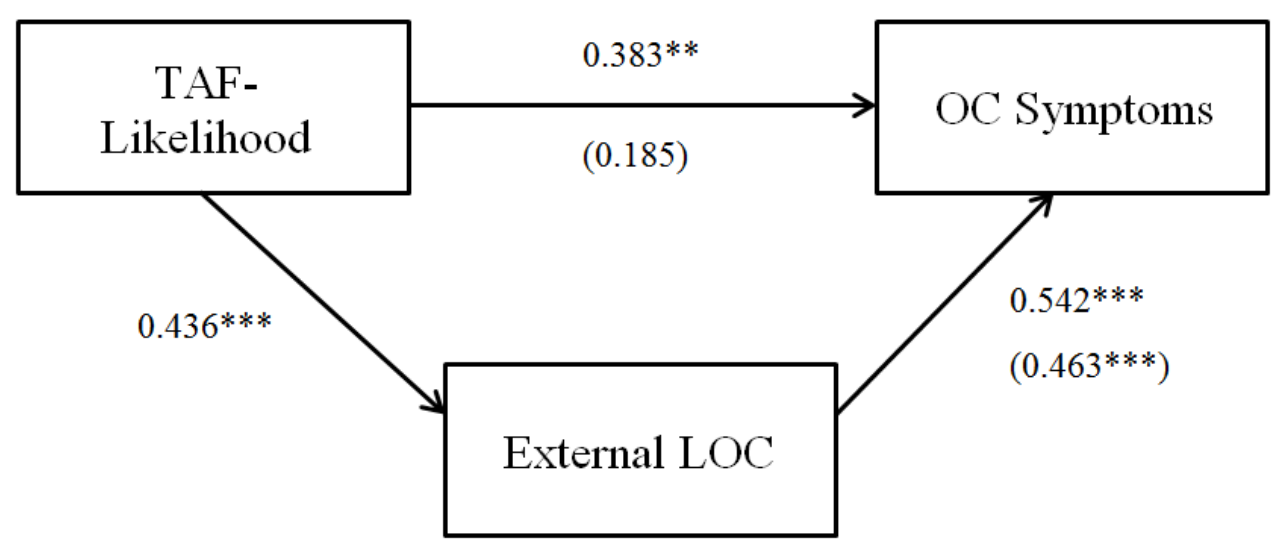

Figure 3.3 A mediational model, with External locus of control mediating between TAF-Likelihood scores and OC symptoms; $* *=p<.05, * * *=p<.001$.

When TAF-moral was entered as the first step of the equation, it was not a significant predictor of OC symptoms. When external locus of control was entered in the second step, it accounted for $28 \%$ of the variance, and was the only significant predictor $(t[65]=4.50, p<.001)$. On the final step, the interaction term for TAFlikelihood and external locus of control did not improve the overall variance accounted for, and was not a significant predictor of overall OC symptoms. Table 3.9

The results of the multiple regression analyses: TAF-moral

\begin{tabular}{llll}
\hline & B & SE B & $\beta$ \\
\hline Step 1 & & & \\
Constant & 54.73 & 8.20 & \\
TAF-moral & .29 & .20 & .19 \\
& & & \\
& & & \\
\hline Step 2 & 15.53 & 11.20 & .10 \\
Constant & .15 & .17 & $.53^{* *}$ \\
TAF-moral & .68 & .15 & \\
External Locus of Control & & & \\
Step 3 & -32.29 & 29.79 & -.11 \\
Constant & -.02 & .01 & \\
Interaction & &
\end{tabular}

Note: $R^{2}=.04$ for Step 1, $\Delta R^{2}=.27(p<.001)$ for Step 2, $\Delta R^{2}=.04(p=.09)$ for Step 3; $*=p<.05, * *=p<.001$. Dependent variable: OC symptoms 


\subsection{Study 2B Discussion}

The goal of Study 2B was to replicate the pattern of results found in study $2 \mathrm{~A}$ within a clinical sample, and with the added variable of thought-action fusion. The results were mixed. Replicating Study 2A, both inflated responsibility and external locus of control were significant predictors of OC symptoms. Unlike the previous study, however, interactive effects of these variables on OC symptom severity were not significant. Thought-Action fusion also predicted OC symptoms, but only when locus of control externality was not included in the regression term. The interaction between TAF and LOC externality also failed to serve as a significant predictor of OC symptom severity however; mediation analyses revealed external locus of control to be a significant mediator of the thought-action fusion and OC symptoms relationship. Taken together, it is clear that locus of control externality plays an important role in the relationship between both types of responsibility beliefs and OC symptomology.

Correlational results revealed that when TAF was broken down into its likelihood and moral components, TAF-likelihood, but not TAF-moral was significantly associated with external locus of control. Similarly, for the regression and mediation analyses, only TAF-likelihood was a significant predictor of OC symptoms. This may be because the target of control beliefs may be very different between these two different cognitive biases. For example, TAF-likelihood is more focused on responsibility for external events/occurrences, so would be more associated with perceived control over events. However TAF-moral may be more associated with perceived control over one's own thoughts, which was not covered in the locus of control measure used in this study. Therefore although in this study, TAFmoral was not associated with control beliefs, if a measure incorporating control beliefs around thoughts was used, this result may be different.

\subsection{General Discussion}

The overall aims of Studies 2A and 2B were to investigate how an external locus of control may work together with responsibility biases to produce symptoms of anxiety. That an external locus of control acted as a mediator between thought-action fusion scores and OC symptoms is in line with previous research examining the mediating role that control beliefs play in the stress and mental health relationship (specifically focused on depression and anxiety). This also supports ideas by Moulding and Kyrios (2006) that it is the discrepancy between perceived control (inflated responsibility and TAF) and sense of control (external locus of control) that 
produces such negative psychological consequences. The results provide support for the theory that the discrepancy between responsibility beliefs and sense of control leads to OC symptoms and distress (Burger, 1992). In terms of OC symptoms, this discrepancy leads individuals to seek to increase their perceived control through compulsions and neutralising techniques (Burger, 1992; Rachman, 1993). These findings further bolster the evidence that targeting inflated responsibility may be important for therapeutic interventions, but that locus of control also plays an important role.

\subsubsection{Clinical Implications}

The combined results of Studies $2 \mathrm{~A}$ and $2 \mathrm{~B}$ have some interesting implications for the treatment of pathological responsibility beliefs. Early research on locus of control described the differences between externals and internals, for example internals tend to be more independent and confident than external and use effective and productive coping strategies like self-monitoring, self-efficacy and selfcontrol; alternatively externals tend to use more maladaptive coping strategies (Strickland, 1989). Techniques aimed at challenging ideas around ideas of personal control may also benefit by teaching adaptive and effective coping strategies. Research focusing on decreasing externality through therapy has found that people can move from having an external locus of control, to becoming more internal, leading to an overall improvement in psychopathology (Lefcourt, 1972).

Contrary to a focus on increasing internality, Moulding and Kyrios (2006) suggest that it may be beneficial to incorporate treatment of faulty control beliefs into Cognitive Behavioural Therapy. They go on to propose teaching clients the strategies to yield control, rather than strategies to regain a sense of control, in response to

dysfunctional responsibility beliefs. The current findings would argue against such a focus on external causes for behaviour as this very externality may exacerbate existing biases in such a way that further reinforces the problem.

\subsubsection{Limitations and Future Research}

The current study focuses solely on responsibility biases, control beliefs and their association with OC symptoms. It would be worthwhile to extend this to more general anxiety and depression symptoms as well, to see whether the emerging patterns are generalizable to other disorders. Results from Study 1 suggest that responsibility beliefs may play an important maintaining role in anxiety and 
depression, so more research in this area will be beneficial for creating effective treatment programs targeting these cognitive biases.

The results of studies $2 \mathrm{~A}$ and $2 \mathrm{~B}$ showed significant associations between responsibility biases and control beliefs. However, from these samples, it is impossible to know the details of this relationship, for example whether one variable precedes or causes the other, or whether they occur concurrently. For more information on these associations, longitudinal research is needed.

\subsection{Chapter Summary}

While thought suppression can be considered a reaction/response to unwanted responsibility beliefs, locus of control contributes to how these beliefs are initially interpreted. The results from Study 2A showed that the interaction of having high levels of inflated responsibility and externality leads to higher OC symptom severity. Results from Study 2B revealed that an external locus of control fully mediated between thought-action fusion and OC symptoms. Locus of control is therefore an important factor in psychopathology when coupled with responsibility biases. The findings add to those of Study 1 and indicate that therapeutic strategies should focus on deflating responsibility to more realistic levels, and to also target and correct beliefs about personal control. 


\section{CHAPTER FOUR}

\section{PATHWAYS TO INFLATED RESPONSIBILITY BELIEFS}

Given the growing body of evidence supporting the important role of responsibility beliefs in the development of psychopathology, it makes sense to investigate their origins and factors which lead to their development. Beck's (1976) cognitive theory of emotional problems described the important role of early childhood and adolescent experiences in forming attitudes which may become dysfunctional later on in the individual's life. Following on from this theory, Salkovskis et al (1999) used information from case reports to propose five primary pathways to the development of inflated responsibility beliefs. Understanding the etiology of responsibility beliefs may lead to important clinical implications. For example, Zucker, Craske, Barrios and Holguin (2002) found that using education intervention techniques in relation to cognitive vulnerability has been found to lead to reductions in psychological symptoms and decreased anxiety. Alternatively, interventions can be targeted at preventing or modifying the development of inflated responsibility beliefs. For example, Coles and Schofield (2008) suggest implementing early prevention programs designed specifically for children in environments characterised by the five pathways described by Salkovskis et al. (1999). The aim of Studies $3 \mathrm{~A}$ and $3 \mathrm{~B}$ was to provide empirical evidence for the pathways theory. This has been conducted once before using an Icelandic translation of a newly developed measure of the pathways (Smari et al., 2010). Thus the current studies are the first to utilise the English version, with both non-clinical and clinical adult participant groups. This is also the first research to include a measure of thought-action fusion as an alternative responsibility bias to examine whether the pathways theory applies here also.

\subsection{Pathways to Inflated Responsibility}

Having a sense of inflated responsibility has been repeatedly found to be a central feature of OCD, and more recently, in other types of psychopathology including generalised anxiety and depression (Rachman, 1998, 2002; Salkovskis, 1985). Given the well-documented significance of inflated responsibility, research examining the etiology of these beliefs is lacking. This information may have implications for conceptualisation, treatment and prevention of anxiety disorders 
(Coles \& Schofield, 2008). As Beck (1976) describes, experiences occurring during childhood and adolescence are crucial to forming potentially dysfunctional attitudes later in life. In an attempt to explain the possible origins of inflated responsibility, Salkovskis et al. (1999) proposed a model of five interacting pathways. These theoretical pathways were developed through years of clinical observations and case studies. It is important to note that these experiences can overlap and co-occur, and of course there are many other factors which may contribute to having an inflated sense of responsibility (Salkovskis et al., 1999). The following factors are hypothesised to be involved in the development of inflated responsibility (Salkovskis et al., 1999, pp. 1060):

1. An early developed and broad sense of responsibility that is deliberately or implicitly encouraged or promoted during childhood.

2. Rigid and extreme codes of conduct and duty.

3. Childhood experiences where sensitivity to ideas of responsibility develops as a result of never being confronted by it.

4. An incident in which one's actions or inaction actually contributed in a significant way to a serious misfortune which affects oneself or others.

5. An incident in which it appeared that one's thoughts and/or actions or inaction contributed to a serious misfortune.

\subsubsection{Heightened Sense of Responsibility}

According to Salkovskis et al. (1999), this pathway is developed early on in childhood, with two possible scenarios. Firstly, some children and adolescents grow up in situations where they assume responsibility for themselves, siblings, and, in some circumstances, parents. Secondly, children grow up in environments where they are repeatedly used as scapegoats for negative occurrences for which they have littleto-no or little control over. Either of these circumstances can result in the child growing up with an overly wide sense of responsibility, leading to a high level of conscientiousness. When individuals with a heightened sense of responsibility find themselves in a situation in which they fail to meet self-imposed standards, they experience strong feelings of failure, disappointment and guilt (Salkovskis, et al., 1999). 


\subsubsection{Rigid and Extreme Codes}

In the same way that parenting style and the home environment can play a role in the development of inflated responsibility, other social influences (e.g., schools and churches) can also impact the development of responsibility beliefs. The second pathway concerns the development of a strict set of personal rules and standards regarding thoughts and behaviours that emerge during childhood and adolescence. Salkovskis et al. (1999) implicate respected authoritarian sources likes schools and clergy, indicating that both types of institutions can teach concepts like blame, guilt, punishment, as well as teachings of the divine, which drives the development of inflated responsibility.

\subsubsection{Overprotection}

The third pathway describes how a child or adolescent may come to develop an inflated sense of responsibility as a result of being raised in an anxious home environment by an overprotective parent or caregiver. Specifically, Salkovskis et al. (1999) describe a scenario in which the parent is excessively anxious and fearful, in particular, worrying about the child's ability to deal with potential dangers, and thus aims to protect them from harm. This, of course, leads to an over-protective parenting style in which responsibility is withheld from the child leading them to become sensitive to responsibility in adulthood.

\subsubsection{Actions contributing to incident}

The fourth pathway describes a situation in which a catastrophic event occurs that affects either the individual or somebody else. Although negative, and potentially traumatic, events occur normally, the important aspect here is that the individual strongly believes that he or she was responsible for the occurrence of the event, or should have done something to prevent it. Salkovskis et al. (1999) also include 'nearmisses' in this category, in which the person believes the misfortune did not occur only because of good luck or unlikely circumstances.

\subsubsection{Perception of actions contributing to incident}

The final pathway is similar to the fourth in that there is some serious incident, however in this case the occurrence of the event is entirely coincidental to the thoughts or behaviours of the individual. Salkovskis et al. (1999) use the example of a child wishing that his or her parent would die, and soon after the parent actually dying. Although the event itself happens by chance, the individual believes that the 
wishes or thoughts make him or her responsible. Of the five pathways, this pathway is most obviously linked to Thought-Action Fusion (Salkovskis et al.).

\subsection{Study 3A}

Until recently, this theory of multiple pathways remained untested. In 2008, Coles and Schofield developed a 23 item, self-report measure to directly assess this theory; the Pathways to Inflated Responsibility Beliefs Scale (PIRBS). As well as developing the measure, Coles and Schofield also had participants complete a measure of OCD symptoms. The results showed a significant correlation between the overall PIRBS score with frequency of OCD symptoms, as well as with all individual PIRBS subscales. This finding provides support for linking the childhood experiences described by Salkovskis et al. (1999) with OC symptoms in later life. However, the pathways theory was described as being pathways to inflated responsibility, not OC symptoms, so it is important to investigate the relationships between pathways and responsibility beliefs, with responsibility beliefs as a mediator between the pathways and OC symptoms.

An Icelandic translation of the PIRBS (Coles \& Schofield, 2008) was tested among 300 undergraduate students in 2010 by Smari et al., who applied a mediational model. Results showed that the total PIRBS scores and each of the subscales correlated significantly with both OC symptoms and inflated responsibility. The results also showed strong support for inflated responsibility as a partial mediator of the relationship between pathways to responsibility and OC symptoms. The lack of a full mediation raises the possibility of the presence and influence of other cognitive variables, for example control beliefs and thought suppression.

The five theoretical pathways to inflated responsibility were also examined by Lawrence and Williams (2011). Sixteen adolescents with a history of OCD were compared to 16 non-clinical adolescent participants on an assessment of the pathways. This involved an original measure of the five pathways proposed by Salkovskis et al. (1999): the Origins Questionnaire for Adolescents (OQA); a semi-structured interview measuring: 1) Broad sense of responsibility; 2) Rigid codes of conduct; 3 )

Shielded from responsibility; 4) Incident associated with negative outcome; and 5) Extremes of responsibility. Results of this research showed that the two groups differed significantly only on one aspect of responsibility beliefs, that is specific incidents related to a negative outcome, with the clinical group reporting higher scores compared to the non-clinical group. However, results found the internal 
consistency of the OQA to be only partly satisfactory. This, in conjunction with a very small sample size makes the findings tentative.

Salkvoskis et al.'s (1999) theory of multiple pathways to inflated responsibility describes a list of childhood experiences that can lead to an individual developing a vulnerability towards inflated responsibility, and OC symptoms. While initial research by Coles and Schofield (2008) has shown promising results in support of this theory, it is important to note that these external experiences are likely to interact with internal, cognitive factors. Whilst many individuals may experience one or more scenarios described in Salkvoskis et al.'s model, only a few may go on to develop inflated responsibility of a clinical level. How one interprets and thinks about their experiences must also play an important role. Studies 3A and 3B will be the first to empirically test the pathways theory within a general adult population as well as an adult clinical population. The aim of this research was to provide empirical support for the Pathways to Inflated Responsibility model and the recently developed measure for this (PIRBS). This has only been done once before by Smari et al. (2010), which was with an Icelandic version. Study 3A will utilise the English translation for the first empirical test of the pathways theory within an adult population.

\subsubsection{Hypotheses}

Based on previous research, it was predicted that: (1) those individuals who show high scores on the PIRBS will demonstrate a higher level of inflated responsibility; (2) that a high overall score on the PIRBS will predict inflated responsibility beliefs; and (3) that each subscale of the PIRBS will be a significant predictor. As the PIRBS and underlying Pathways theory were devised as predictors of inflated responsibility in relation to OCD, a measure of Obsessive Compulsive symptoms was also included. Similar to the results of Smari et al. (2010), it was also predicted that (4) inflated responsibility would partially mediate the relationship between scores on the PIRBS and OC symptoms.

\subsection{Method}

\subsubsection{Participants}

The participants for this study were 265 individuals from the same pool as that for Study 2A who completed an online survey. Participants were recruited using a snowballing effect through the social networking site Facebook. The sample consisted of 149 females (56.2\%) and 45 males (17\%); 71 did not provide this information 
(26.8\%). The participants ages ranged from 16 to 66 with a mean age of 28.23 $(\mathrm{SD}=10.69)$. The sample consisted mainly of New Zealand European participants (30.9\%), 3.4\% were Asian, with the remaining participants being European (14\%), Maori (2.3\%), Pacific Nations (0.8\%), American (12.8\%), Australian (7.2), and the remaining being other/refused.

\subsubsection{Measures}

The Obsessive Compulsive Inventory - Revised (OCI-R; Foa et al., 2002) and the Responsibility Attitude Scale (RAS; Salkovskis et al., 2000) were used in this study and are described in detail in Study 1 (Chapter 2). Additionally, the Pathways to Inflated Responsibility Beliefs Scale (PIRBS; Coles \& Schofield, 2008) is a 23 item scale recently devised to measure four pathways to inflated responsibility. The scale has four subscales: Heightened Responsibility (5 items, e.g. 'I was responsibility for keeping our house functioning smoothly'); Overprotection (5 items, e.g. 'My parent(s) thought that I was unable to deal with danger'); Rigid Rules (5 items, e.g. 'Adults around me strictly enforced rules'); and Actions Caused/Influenced (8 items, e.g. 'I am confident that something I did resulted in someone else experiencing a serious misfortune). For each item the participant rated the statements for how frequently they were true for them, from 0 ('never') to 4 ('always'), with higher overall scores indicating higher endorsement. While there are five theoretical pathways, this measure combines the last two into one subscale. A copy of this scale is in Appendix K.

\subsubsection{Procedure}

The questionnaire data was collected via an anonymous online survey using Survey Monkey. The initial sample of participants was invited to complete the survey by the principle investigator via connections on the Facebook social networking site. Subsequent participants were invited by those who had already completed the survey, or were those who randomly came across the site, using a snowballing effect. The survey itself took approximately 20 minutes to complete. The Victoria University Human Ethics Committee gave approval for this study prior to its beginning. The information sheet, questionnaire and the debriefing sheet are included in Appendix B. 


\subsection{Results}

\subsubsection{Descriptive Statistics}

The means, standard deviations and reliability coefficients for all variables are shown in Table 4.1. All scales (and subscales of the PIRBS) showed good to excellent reliability.

Table 4.1

Means, standard deviations and reliability.

\begin{tabular}{lllc}
\hline & M & SD & $\alpha$ \\
\hline OCI & 36.91 & 13.59 & .92 \\
RAS & 69.38 & 33.86 & .91 \\
PIRBS - Overall & 38.02 & 15.12 & .88 \\
\multicolumn{1}{l}{ PIRBS - Heightened Responsibility } & 6.07 & 4.75 & .84 \\
PIRBS - Rigid Rules & 11.43 & 4.60 & .89 \\
PIRBS - Actions & 10.04 & 8.03 & .92 \\
PIRBS - Overprotection & 8.61 & 4.51 & .81
\end{tabular}

Note: $\alpha$ : Cronbach's alpha; OCI-R: Obsessive Compulsive Inventory - Revised (Foa et al., 2002 RAS: Responsibility Attitude Scale (Salkovskis et al., 2000); PIRBS:

Pathways to Inflated Responsibility Scale and 4 subscales (Coles \& Schofield, 2008).

Pearson Correlations for all variables are shown in Table 4.2. The overall PIRBS total, as well as each of the four subscales all correlated significantly with OC symptoms and inflated responsibility. All subscales were significantly correlated with the overall PIRBS score, and with each other, apart from Overprotection and Rigid Rules. 
Table 4.2

Pearson correlations

\begin{tabular}{lllllll}
\hline & 1 & 2 & 3 & 5 & 6 & 7 \\
\hline (1) OCI & 1 & & & & & \\
(2) RAS & $.322^{* *}$ & 1 & & & & \\
(3) PIRBS & $.521 * *$ & $.390^{* *}$ & 1 & & & \\
(4) H Resp & $.201 * *$ & $.174 * *$ & $.512 * *$ & 1 & & \\
(5) Rigid Rules & $.242 * *$ & $.252 * *$ & $.252 * *$ & $.282 * *$ & 1 & \\
(6) Actions & $.541^{* *}$ & $.406 * *$ & $.802 * *$ & $.271 * *$ & $.218^{* *}$ & 1 \\
(7) Overprotect & $.268^{* *}$ & $.190 * *$ & $.588^{* *}$ & -.108 & $.325 * *$ & $.317 * *$ \\
\hline
\end{tabular}

Note: $* * p<.01$. OCI-R: Obsessive Compulsive Inventory - Revised (Foa et al., 2002 RAS: Responsibility Attitude Scale (Salkovskis et al., 2000); PIRBS: Pathways to Inflated Responsibility Scale (Coles \& Schofield, 2008).

\subsubsection{Regression Analyses}

The overall PIRB scale was a significant predictor of inflated responsibility $(t[169]=5.49, p<.001)$. Regression was used to assess whether the four individual subscales were also predictors. Variables were entered individually, and results can be seen in Table 4.3. Results showed that all of the subscales were significant predictors of inflated responsibility.

Table 4.3

Individual Regression Analyses: Four subscales of the PIRBS

\begin{tabular}{lccc}
\hline & $B$ & $S E B$ & Beta \\
Heightened Responsibility & -1.270 & .542 & $-.174^{*}$ \\
Rigid Rules & -1.816 & .524 & $-.252^{* *}$ \\
Actions & -1.680 & .283 & $-.406^{* *}$ \\
Overprotection & -1.426 & .552 & $-.190^{*}$ \\
\hline $\begin{array}{l}\text { Dependent Variable: Inflated Responsibility } \\
\text { Note: } * * p<.001, * p<.05 ; \text { PIRBS: Pathways to Inflated Responsibility Scale (Coles } \\
\text { \& Schofield, 2008). }\end{array}$
\end{tabular}




\subsubsection{Mediation Analyses}

The mediation analysis was performed using Medgraph (Jose, 2003). In order for a mediational model to apply, all three variables (the mediator, the independent variable, and the dependent variable) must be significantly correlated (Baron \& Kenny, 1986). As can be seen in Table 4.2, inflated responsibility (the mediator), PIRBS scores (the independent variable), and OC symptoms (the dependent variable) were all interrelated, satisfying this condition. Secondly, there needs to be less of an association between the predictor and the outcome when the mediator is controlled for. A Sobel test was conducted in order to test for the indirect effect. A value of $z=$ 4.18 was found $(p<.001)$, meaning a significant, full mediation was found (see Figure 4.1).

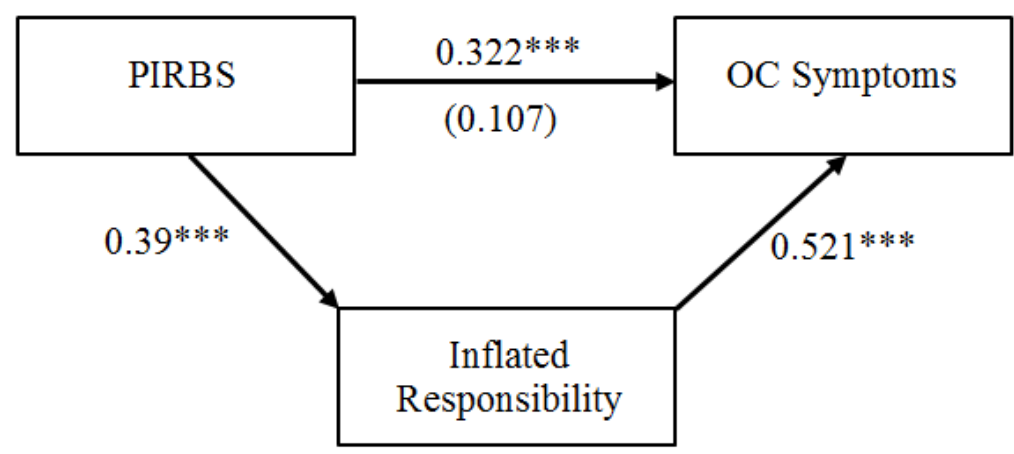

Figure 4.1 A mediational model, with Inflated Responsibility mediating between PIRBS scores and OC symptoms. ${ }^{* *}=p<.05, * * *=p<.001$.

\subsection{Study 3A Discussion}

Study 3A aimed to investigate the influence of early experiences (measured using the PIRBS) on the development of inflated responsibility in a normal adult population. Good to excellent reliability coefficients were found for the overall scale, as well as the four subscales. Overall scores on the PIRBS were significantly correlated with inflated responsibility. Each of the four subscales also correlated significantly with inflated responsibility. The obtained results showed that the overall PIRBS scores, as well as those of the four subscales all significantly correlated with obsessive compulsive symptoms. Regression analyses revealed that the overall PIRBS score, as well as all of the individual pathways were significant predictors of inflated responsibility. 
The strongest results, both correlations and regressions, were for the pathway combining situations in which one's action/inaction caused/influenced misfortune (that relating to the fourth and fifth theoretical pathways). This supports research by Lawrence and Williams (2011), who suggest that incidents involving real or imagined responsibility for negative events and/or coincidental events where the individual feels to blame, can alone create an inflated sense of responsibility. Such 'critical incidents' would not necessarily trigger obsessional/compulsive symptoms alone, but may combine with pre-existing beliefs to trigger and maintain inflated responsibility (Salkovskis et al., 1999).

Results also showed that Inflated Responsibility played a full mediatory role between PIRBS and OC symptoms. These results are similar to Smari et al. (2010), who found a partial mediation. This provides support for the theory proposed by Smari et al., that if inflated responsibility leads to OC symptoms, and higher endorsement on the pathways is associated with higher inflated responsibility, then the latter should mediate between the former and OC symptoms. These results also provide empirical support for Salkovskis et al.'s (1999) theory of multiple pathways leading to inflated responsibility.

\subsection{Study 3B}

The aim of Study 3B was to replicate Study 3A with an adult clinical population, and to include an examination of the pathways theory in relation to TAF as an alternative to inflated responsibility. A measure of TAF was included to examine whether it was predicted by any of the PIRBS subscales, similar to inflated responsibility. The items measuring the Actions subscale refer to situations where an individual believes that something they did (or did not do) contributed to a negative occurrence (e.g. 'I am confident that something I did resulted in someone else experiencing a serious misfortune'). Additionally in the instructions for this section, it states "sometimes it appears that something we think or do may have results in a serious misfortune" (PIRBS; Coles \& Schofield, 2008). This links directly to the concept of TAF-likelihood where one believes their thoughts may lead to a negative event (Abramowitz, et al., 2003). Indeed, Salkovskis et al. (1999) stated that people who are more prone to TAF biases are most likely to experience the type of responsibility beliefs measured by the Actions subscale. The items measuring the Rigid Rules subscale however, seem to be more related to the concept of TAF-moral. 
These items refer to underlying personal standards and rules, often developed through association with strict parental codes, schools and churches (Abramowitz, et al., 2003), ideas often included in discussions of TAF-moral. Examples from this subscale include "I was taught that rules were to be obeyed without discussion" and "My parent(s) strongly valued obedience".

\subsubsection{Hypotheses}

Similar to Study 3A, it was predicted that: (1) higher total scores on the PIRB scale would be related to higher inflated responsibility. Based on theory (Salkovskis et al., 1999), it was expected that: (2) the four subscales of the PIRBS would be predictive of inflated responsibility. It was also predicted that: (3) the overall PIRBS would be predictive of thought-action fusion. Additionally, when breaking the TAF concept down into TAF-moral and TAF-likelihood, it is predicted that: (4) the Actions subscale of the PIRBS would be predictive of TAF-likelihood, while (5) the Rigid Rules subscale would be predictive of TAF-moral. In terms of mediation, similar results to Study 3A were expected, (6) where each of the responsibility biases (inflated responsibility, TAF, TAF-moral and TAF-likelihood) would play a mediating role between PIRBS and OC symptoms.

\subsection{Method}

\subsubsection{Participants}

The participants for this study are from the same pool as Study 2B, and were 67 individuals who were all seeking and receiving treatment for anxiety disorders. Participants completed an online survey, taking approximately 15-20 minutes. Of the participants, $28.4 \%$ were diagnosed with OCD, 26.9\% with Generalised Anxiety Disorder, $28.4 \%$ with Social Anxiety, $3 \%$ with Panic Disorder, $1.5 \%$ with Agoraphobia, $7.5 \%$ with some other anxiety disorder, and $4.3 \%$ did not say. Males made up $43.1 \%$, and females made up $56.9 \%$ of the group. The mean age was 40.69 $(\mathrm{SD}=12.7)$ years, ranging from 18 to 67 .

\subsubsection{Measures}

The measures for Study 3B consisted of the Pathways to Inflated Responsibility Beliefs Scale (PIRBS; Coles \& Schofield, 2008), the Obsessive Compulsive Inventory - Revised (OCI-R; Foa et al., 2002) the Responsibility Attitude 
Scale (RAS; Salkovskis et al., 2000), and the Thought-Action Fusion Scale-Revised (TAF-R; Shafran et al., 1996).

\subsubsection{Procedure}

Individuals were invited to participate in the study via an email sent to clients of the Anxiety Clinic in Canterbury. The questionnaire data was collected via an anonymous online survey using Qualtrics software (Qualtrics, 2013). The Central Region Ethics Committee of the New Zealand Ministry of Health gave approval for this study prior to its beginning. The information sheet, questionnaire and the debriefing sheet are included in Appendix C.

\subsection{Results}

\subsubsection{Descriptive Statistics}

The means, standard deviations and reliability coefficients for all variables are shown in Table 4.4. All scales (and subscales of the PIRBS) showed good to excellent reliability.

Table 4.4

Means, standard deviations and reliability.

\begin{tabular}{lllc}
\hline & M & SD & $\alpha$ \\
\hline OCI & 66.63 & 18.57 & .91 \\
RAS & 139.78 & 32.55 & .97 \\
PIRBS - Overall & 75.00 & 15.90 & .91 \\
$\quad$ PIRBS - Heightened Responsibility & 13.87 & 4.76 & .87 \\
$\quad$ PIRBS - Rigid Rules & 18.71 & 4.35 & .92 \\
\multicolumn{1}{c}{ PIRBS - Actions } & 26.92 & 9.52 & .96 \\
$\quad$ PIRBS - Overprotection & 16.00 & 5.13 & .90 \\
TAF - Overall & 64.74 & 19.02 & .95 \\
TAF - Moral & 39.44 & 12.77 & .94 \\
TAF - Likelihood & 25.10 & 9.28 & .96 \\
\hline
\end{tabular}

Note: $\alpha$ : Cronbach's alpha; Obsessive Compulsive Inventory - Revised (Foa et al., 2002); RAS: Responsibility Attitude Scale (Salkovskis et al., 2000); PIRBS:

Pathways to Inflated Responsibility Scale and 4 subscales (Coles \& Schofield, 2008); TAF: Thought-Action Fusion Scale Revised (Shafran et al., 1996). 
Pearson Correlations for all variables are shown in Table 4.5. The overall PIRBS total, correlated significantly with OC symptoms, Inflated Responsibility and Thought-Action Fusion, as well as the Moral and Likelihood subscales of TAF.

Table 4.5

Pearson correlations

\begin{tabular}{llllllllll}
\hline & 1 & 2 & 3 & 4 & 5 & 6 & 7 & 8 & 9 \\
\hline (1) OCI & 1 & & & & & & & & \\
(2) RAS & $.469^{* *}$ & 1 & & & & & & & \\
(3) TAF & $.317^{*}$ & $.327^{*}$ & 1 & & & & & & \\
(4) TAF-M & .193 & .145 & $.910^{* *}$ & 1 & & & & & \\
(5) TAF-L & $.383^{* *}$ & $.458^{* *}$ & $.813^{* *}$ & $.497^{* *}$ & 1 & & & & \\
(6) PIRBS & $.392^{* *}$ & $.517^{* *}$ & $.607^{* *}$ & $.467^{* *}$ & $.592^{* *}$ & 1 & & & \\
(7) HR & $.326^{*}$ & $.377^{* *}$ & $.324^{*}$ & .251 & $.280^{*}$ & $.724^{* *}$ & 1 & & \\
(8) RR & .022 & .067 & .181 & $.333^{* *}$ & -.022 & $.370^{* *}$ & .212 & 1 & \\
(9) AS & $.394^{* *}$ & $.409^{* *}$ & $.524^{* *}$ & $.296^{*}$ & $.621^{* *}$ & $.763^{* *}$ & $.472^{* *}$ & -.226 & \\
(10) OP & .092 & -.076 & $.335^{* *}$ & $.339^{* *}$ & $.266^{*}$ & $.678^{* *}$ & .225 & $.538^{* *}$ & .217 \\
\hline
\end{tabular}

Note: $* * p<.01,{ }^{*} p<.05$. OCI: Obsessive Compulsive Inventory - Revised (Foa et al., 2002); RAS: Responsibility Attitude Scale (Salkovskis et al., 2000); TAF: ThoughtAction Fusion Scale Revised with 2 subscales: TAF-M (TAF-moral), TAF-L (TAFlikelihood; Shafran et al., 1996); PIRBS: Pathways to Inflated Responsibility Scale with 4 subscales: HR (Heightened Responsibility), RR (Rigid Rules), AS (Actions), OP (Overprotection; Coles \& Schofield, 2008).

\subsubsection{Inflated Responsibility}

\subsubsection{Regression Analyses}

The overall PIRB scale was a significant predictor of inflated responsibility $(t[66]=4.18, p<.001)$. Regression was used to assess whether each of the four subscales were also predictors. Variables were entered individually, and results can be seen in Table 4.6. Results showed that the Heightened Responsibility and Actions subscales were all significant predictors of inflated responsibility. 
Table 4.6

Individual Regression Analyses: Four subscales of the PIRBS on Inflated Responsibility

\begin{tabular}{lccc}
\hline & $B$ & $S E B$ & Beta \\
Heightened Responsibility & 2.825 & .896 & $.385^{*}$ \\
Rigid Rules & 1.059 & .964 & .140 \\
Actions & 1.693 & .401 & $.485^{* *}$ \\
Overprotection & .954 & .850 & .145 \\
\hline
\end{tabular}

Dependent Variable: Inflated Responsibility

Note: $* p<.05, * * p<.001$; PIRBS: Pathways to Inflated Responsibility Scale (Coles $\&$ Schofield, 2008).

\subsubsection{Mediation Analyses}

As can be seen in Table 4.5, inflated responsibility (the mediator), PIRBS scores (the independent variable), and OC symptoms (the dependent variable) were all significantly correlated, satisfying the first condition of Baron and Kenny (1986). Using Medgraph (Jose, 2003), a Sobel test was conducted in order to test for the indirect effect. A value of $z=2.91$ was found ( $p=.004)$, meaning a significant, full mediation was found (see Figure 4.2).

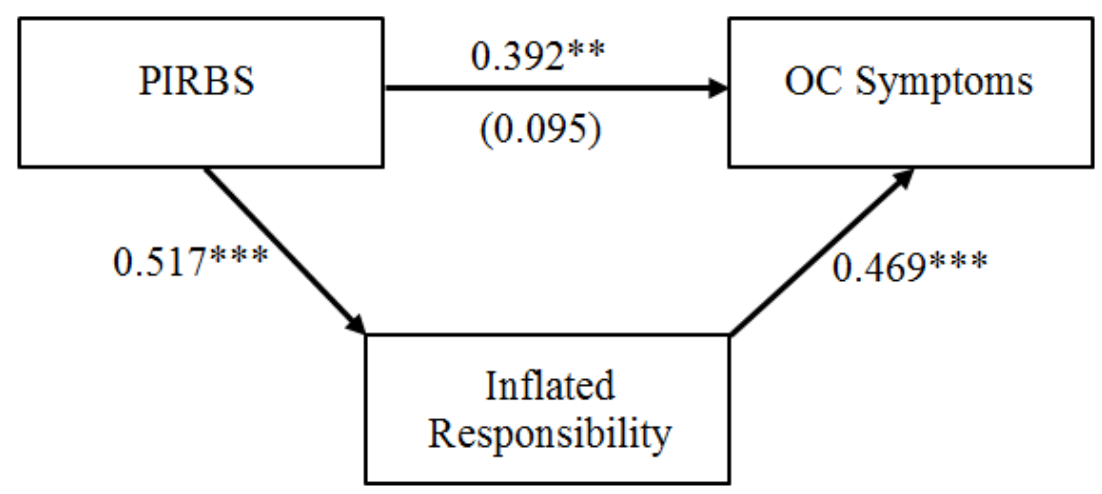

Figure 4.2 A mediational model, with Inflated Responsibility mediating between PIRBS scores and OC symptoms. $* *=p<.05, * * *=p<.001$.

\subsubsection{Thought-Action Fusion}

\subsubsection{Regression Analyses}

The overall PIRB scale was also a significant predictor of thought-action fusion $(t[66]=5.61, p<.001)$. Regression was used to assess whether the four 
subscales were also predictors. Variables were entered individually, and results can be seen in Table 4.7. Results showed that only the Actions and Overprotection subscales were significant predictors of thought-action fusion.

Table 4.7

Individual Regression Analyses: Four subscales of the PIRBS on Thought-Action Fusion

$B \quad S E B \quad$ Beta

$\begin{array}{lccc}\text { Heightened Responsibility } & 1.274 & .497 & .324 \\ \text { Rigid Rules } & .779 & .552 & .181 \\ \text { Actions } & .978 & .211 & .524 * * \\ \text { Overprotection } & 1.223 & .452 & .335^{*}\end{array}$

Dependent Variable: Thought-Action Fusion

Note: ${ }^{*} p<.05, * * p<.001$; PIRBS: Pathways to Inflated Responsibility Scale (Coles $\&$ Schofield, 2008).

\subsubsection{Mediation Analyses}

As can be seen in Table 4.5, TAF (the mediator), PIRBS scores (the independent variable), and OC symptoms (the dependent variable) were all significantly correlated, satisfying the first condition of Baron and Kenny (1986). Using Medgraph (Jose, 2003), a Sobel test was conducted in order to test for the indirect effect. A value of $z=1.37$ was found ( $p=.17)$, meaning no significant mediation was found (see Figure 4.3).

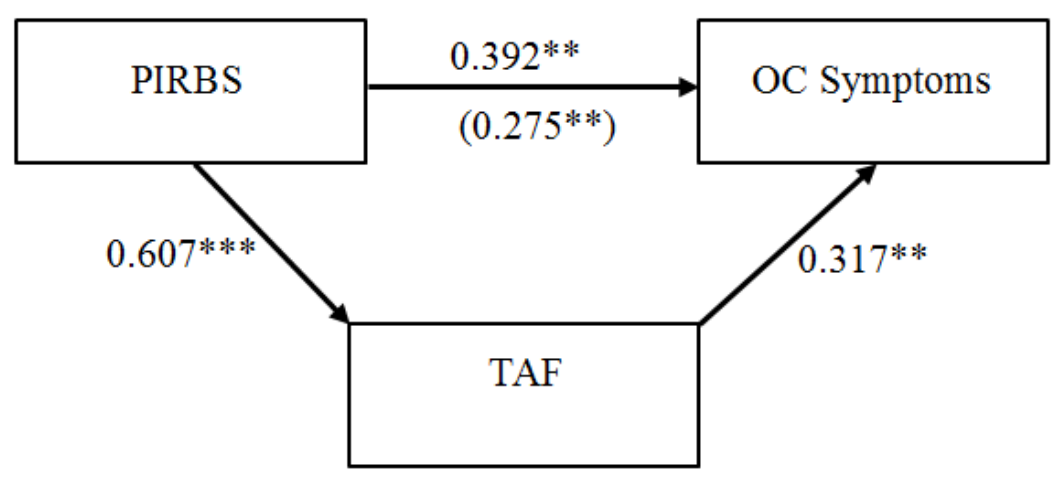

Figure 4.3 A (non significant) mediational model, with TAF mediating between PIRBS scores and OC symptoms. ${ }^{* *}=p<.05, * * *=p<.001$. 


\subsubsection{Thought-Action Fusion - Likelihood}

\subsubsection{Regression Analyses}

The overall PIRB scale was also a significant predictor of thought-action fusion - likelihood $(t[66]=5.64, p<.001)$. Regression was used to assess whether the four subscales were also predictors. Variables were entered individually, and results can be seen in Table 4.8. Results showed that the Heightened Responsibility, Actions and Overprotection subscales were significant predictors of TAF-likelihood.

Table 4.8

Individual Regression Analyses: Four subscales of the PIRBS on Thought-Action Fusion-Likelihood

\begin{tabular}{lccc}
\hline & $B$ & $S E B$ & Beta \\
Heightened Responsibility & .546 & .240 & $.280^{*}$ \\
Rigid Rules & -.046 & .265 & -.022 \\
Actions & .589 & .094 & $.621^{* *}$ \\
Overprotection & .480 & .219 & $.266^{*}$ \\
\hline $\begin{array}{l}\text { Dependent Variable: TAF-likelihood } \\
\text { Note: } * p<.05, * * p<.001 ; \text { PIRBS: Pathways to Inflated Responsibility Scale (Coles } \\
\& \text { Schofield, 2008). }\end{array}$
\end{tabular}

\subsubsection{Mediation Analyses}

As can be seen in Table 4.5, TAF-likelihood (the mediator), PIRBS scores (the independent variable), and OC symptoms (the dependent variable) were all significantly correlated, satisfying the first condition of Baron and Kenny (1986). Using Medgraph (Jose, 2003), a Sobel test was conducted in order to test for the indirect effect. A value of $z=2.04$ was found ( $p=.04$ ), meaning a significant, full mediation was found (see Figure 4.4). 


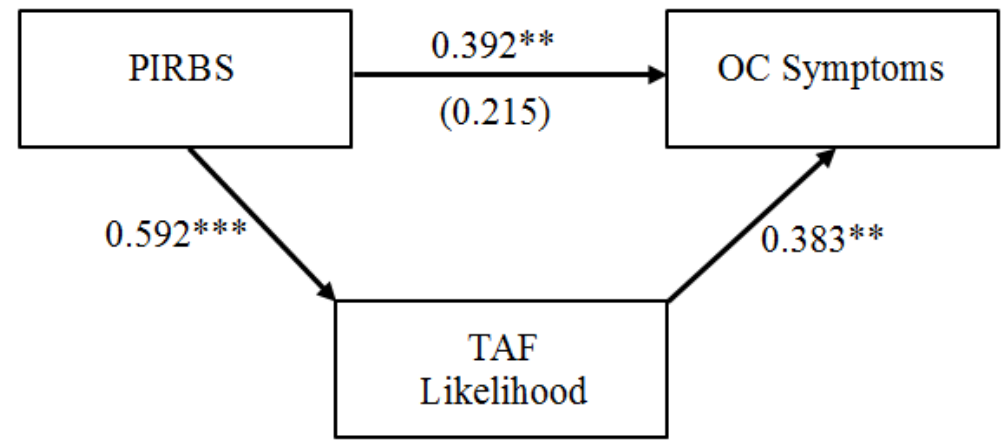

Figure 4.4 A mediational model, with TAF-likelihood mediating between PIRBS scores and OC symptoms. $* *=p<.05, * * *=p<.001$.

\subsubsection{Thought-Action Fusion - Moral}

\subsubsection{Regression Analyses}

The overall PIRB scale was also a significant predictor of thought-action fusion - moral $(t[66]=3.89, p<.001)$. Regression was used to assess whether the four subscales were also predictors. Variables were entered individually, and results can be seen in Table 4.9. Results showed that the Rigid Rules, Actions and Overprotection subscales were significant predictors of TAF-moral.

Table 4.9

Individual Regression Analyses: Four subscales of the PIRBS on Thought-Action Fusion-Moral

\begin{tabular}{lccc}
\hline & $B$ & $S E B$ & Beta \\
Heightened Responsibility & .667 & .343 & .251 \\
Rigid Rules & .969 & .357 & $.333^{*}$ \\
Actions & .378 & .162 & $.296^{*}$ \\
Overprotection & .837 & .305 & $.339^{*}$ \\
\hline
\end{tabular}

Dependent Variable: TAF-moral

Note: $* p<.05$, ** $p<.001$; PIRBS: Pathways to Inflated Responsibility Scale (Coles $\&$ Schofield, 2008).

\subsubsection{Mediation Analyses}

Mediation analyses were not able to be performed with TAF-moral as there was no significant correlation between OC symptoms and TAF-moral. 


\subsection{Study 3B Discussion}

Study 3B aimed to investigate the influence of early experiences (measured using the PIRBS) on the development of inflated responsibility and thought-action fusion in an adult, clinical population. In terms of inflated responsibility, overall scores on the PIRBS were found to be significantly predictive, as well as the Heightened Responsibility and Actions subscales. Overall PIRBS scores were also significantly predictive of Thought-Action Fusion, with the Actions and Overprotection subscales being significant. When Thought-Action Fusion was dichotomised into Likelihood and Moral, the hypothesis was supported for Likelihood, with the Actions subscale being a significant predicting subscale, as well as Heightened Responsibility and Overprotection. For Moral, the Rigid Rules, Actions and Overprotection subscales were significant predictors. Correlative results revealed that the overall PIRBS was significantly associated with both inflated responsibility and thought-action fusion (including both moral and likelihood subscales).

The results also showed that inflated responsibility played a full mediating role between PIRBS and OC symptoms, as was found in Study 3A, providing further support for Salkovskis et al.'s (1999) theory. This was also the case for TAFlikelihood; however, there was no mediation for overall TAF, and TAF-moral. A mediation analysis was not performed for TAF-moral, as it did not significantly correlate with OC symptoms.

\subsection{General Discussion}

The overall aims of Studies 3A and 3B were to investigate the roles of childhood/early experiences in the etiology of responsibility biases using both clinical and non-clinical participant samples, and to provide further support for the newly developed PIRBS (Coles \& Schofield, 2008) measure. These studies were the first to empirically test the pathways theory with an adult, English speaking sample, and with an adult clinical sample. Study 3B was also the first study to apply the theoretical pathways to TAF, as an alternative responsibility bias. In terms of the PIRBS, for both the clinical and non-clinical samples, the overall scale and each of the four subscales all showed good to excellent reliability. Overall regression analyses revealed that for the non-clinical sample, each of the four subscales were significant predictors of inflated responsibility. For the clinical sample, Heightened Responsibility and Actions were significant predictors of inflated responsibility. Thought-Action Fusion was only 
measured in the clinical sample and results revealed that Actions and Overprotection were both significant predictors. When TAF was broken down into different types, TAF-moral was predicted by Rigid Rules, Actions and Overprotection, whereas TAFlikelihood was significantly predicted by the Heightened Responsibility, Actions and Overprotection subscales. Overall results for Inflated Responsibility provided empirical support for Salkovskis et al.'s (1999) theory of multiple pathways, and support for the recently developed PIRBS (Coles \& Schofield, 2008) measure.

From these overall results, it can be seen that for both participant samples and for all types of responsibility biases measured, the Actions subscale was the only common, significant predictor. This subscale aims to measure the fourth and fifth pathways from Salkovskis et al.'s (1999) original theory, in which one's thoughts and/or actions actually contributed to a negative incident, or it appears as though one's thoughts and/or actions contributed to a negative incident. This finding is similar to the research by Lawrence and Williams (2010) who found the only difference on the five theoretical pathways between adolescents with and without OCD was those in the OCD group showed a greater sense of responsibility for specific incidents. These pathways differ from the other three developmental pathways (Heightened Responsibility, Overprotection and Rigid Rules) in a number of ways. Firstly, the Actions pathway describes a sudden onset of inflated responsibility, whereas the other pathways tend to be more gradual and cumulative. Secondly, this occurs after a critical incident (or even a near-miss incident), with a relatively later onset, while the other theoretical pathways evolve from many accumulating experiences throughout early life that individually have little effect on responsibility beliefs (Salkovskis, et al.). Finally, Salkovskis et al. suggest that the inflated beliefs originating from such critical incidents tend to be a lot more specific and circumscribed, rather than being generalised to other aspects of daily life.

The results also provided support for the idea proposed by Salkovskis et al. (1999) that endorsement of the pathways theory is associated with high inflated responsibility, and that inflated responsibility mediates between the pathways and OC symptoms. For both the non-clinical and clinical samples, a significant full mediation was found. When this model was applied to TAF, a full mediation was only found for TAF-likelihood. The lack of a mediation for TAF-moral was due to there being no significant correlation with OC symptoms in the clinical sample. This supports previous research such as that by Coles, Mennin and Heimberg (2001) that TAF- 
likelihood has a closer relationship with OCD than TAF-moral, which seems to be more related to depression.

\subsubsection{Clinical Implications}

The combined results of Studies 3A and 3B have some interesting implications for the treatment of responsibility beliefs within psychopathology, however these may be somewhat limited. As Salkovskis et al. (1999) discuss, more progress is made in therapy when focusing on maintaining factors, rather than identifying the origins of belief biases. In saying this, from the proposed pathways, the authors also identified the final two pathways (those measured by the Actions subscale of the PIRBS), as being the most amenable to change through cognitive behavioural therapy. This may be because the Actions pathway describes key events/critical incidents, rather than responsibility biases evolving from a long process of many small experiences. Having a target source of origin of where cognitive biases come from means that therapy can be focussed on replacing incorrect thoughts around the incident, potentially deflating the exaggerated sense of responsibility generally. Ideally, this would be possible early on, before the beliefs become fixed in the individual's belief system (Salkovskis et al.).

Additionally, the results support the idea of early intervention for potentially preventing subsequent problematic responsibility beliefs. Firstly, in reference to the Actions pathway measuring critical incidents, targeted programs may be developed for assessing the risks of developing responsibility biases. For example, following an accidental death, the way in which a family member describes the occurrence may signal the need for cognitive restructuring, which may help to reduce longer-lasting inflated responsibility beliefs and related symptoms anxiety/depression. Especially for children, it may be appropriate to include discussing these ideas as part of immediate trauma and victim support counselling, in an effort to avoid this becoming a long-term problem. Secondly, Coles and Schofield (2008) suggest that once more is known on the etiology of responsibility beliefs, this knowledge can be used for developing prevention programs. Children who are identified as being in vulnerable environments (such as living with Rigid Rules, or have Heightened Responsibility e.g. living in a single parent home), as well as at risk for anxiety and/or depression, may be able to participate in specifically designed programs for targeting responsibility beliefs. 


\subsubsection{Limitations}

The main limitation with Studies $3 \mathrm{~A}$ and $3 \mathrm{~B}$ is that the data on childhood experiences was collected retrospectively, and participants may have been providing information from well over thirty years ago. Consequently the data may not be an entirely accurate portrayal of events. Similarly, those participants who are in treatment for anxiety disorders may show some responsibility biases in their memory of past events. Lawrence and Williams (2010) describe how particularly with the Actions subscale, memories of how responsible the individual felt at the time of the critical incident may be affected by anxiety symptoms; they suggest that participants would report a higher level of responsibility when experiencing elevated anxiety symptoms. Therefore caution is recommended when interpreting this kind of retrospective data.

\subsubsection{Future Research}

The most effective way to investigate the etiology of responsibility beliefs, and to thoroughly examine the Pathways to Inflated Responsibility theory (Salkovskis et al., 1999) is to conduct longitudinal research. Due to the correlational nature of the current research, extrapolation of a causal pathway would be unwise. Another avenue to pursue in terms of the origins of responsibility beliefs is investigating the factors that may interact with the theoretical pathways. Salkovskis et al. (1999) identified some of these factors as criticism and blame, life events, prolonged stress, depressed mood, and situational increases in responsibility (e.g. having a baby or a work promotion). Although the theory of developmental pathways to inflated responsibility describes common patterns, it is more likely that it is the result of complex interactions of many variables and experiences that lead to responsibility biases (Alloy \& Riskind, 2006). Identifying some of these may help to further our knowledge of how these biases develop, and therefore create more targeted interventions and treatments.

\subsection{Chapter Summary}

Studies 3A and 3B aimed to empirically test the theoretical work of Salkovskis et al.'s (1999) pathways to inflated responsibility within both adult clinical and nonclinical samples. Study 3B was the first to examine the pathways theory using the PIRBS (Coles \& Schofield, 2008) with an adult, clinical population. Additionally, this was the first research to include thought-action fusion in the clinical sample to shed light on the similarities in etiology between the two different responsibility biases, 
and whether any of the theoretical pathways were predictive of TAF. These findings provide support for Salkovskis et al.'s theory of multiple pathways to inflated responsibility, highlighting the importance of early childhood events on the later development of dysfunctional responsibility beliefs in adulthood. Results from both Studies 3A and 3B found that inflated responsibility played a full mediating role between endorsement of the pathways and OC symptoms. This was also found with TAF-likelihood in the clinical sample. The overall results identify critical incidents where one actually does have influence on a negative occurrence, or where it appears as so to the individual, can be predictive of later developing inflated responsibility beliefs and TAF-likelihood. 


\section{CHAPTER FIVE}

\section{RELIGIOUS AFFILIATION AND RESPONSIBILITY BELIEFS}

The collective results of Studies 1,2A and 2B, 3A and 3B have shown the importance of both TAF and inflated responsibility in psychopathology, namely symptoms of OCD, anxiety and depression. Existing research on these responsibility biases tends to consider them as maladaptive constructs, and indicative of psychopathology. The aim of Study 4 was to consider whether these responsibility biases can exist outside the realm of psychopathology, so that they are not always indicative of underlying psychological symptoms. This has important implications for treatment, as although it is clear that both TAF and inflated responsibility are associated with particular disorders like OCD, depression and anxiety, caution should be taken when making assumptions and pathologising beliefs that may be acceptable in some cultural and religious groups (Siev \& Cohen, 2007).

\subsection{Literature Review}

Religiosity has been repeatedly linked with both thought-action fusion (especially the moral component) and inflated responsibility (e.g. Rassin \& Koster, 2003; Berle \& Starcevic, 2005). This does not, of course, mean that all individuals with strong religious beliefs will also develop thought-action fusion. Abramowitz, Deacon, Woods and Tolin (2004) argue that pre-existing cognitive biases like inflated responsibility may predispose one to affiliate more strongly with their religious beliefs and teachings. Similarly, individuals who already score highly on TAF may go on to become more involved in religion. It remains unclear whether religious teachings (or one's interpretations of these) can lead to TAF beliefs, and/or whether those who are already endorsing such beliefs are attracted to religion. In the end, the relationship between religiosity and responsibility beliefs is likely to be a complex and multiply-determined relationship taking into account internal (i.e., personality, biases, behavioural tendencies), and external (i.e., specific religious teachings, childhood experiences) factors (Abramowitz et al., 2004; Salkovskis et al., 1999).

Most previous research in this area has been conducted by examining differences between Christians and Jews. For example Cohen and Rozin (2001) investigated how Jews and Protestants moralised mental states. That is, believing that one's thoughts have moral importance, similar to the moral component of TAF. By using hypothetical vignettes, they found that both participant groups assigned equal 
moral significance to particular behaviours, but that Protestants considered thoughts to be more morally important that Jews. Following on from this research, Cohen (2003) conducted three studies to look at how Jews and Protestants differ in whether they consider: (a) thoughts about immoral actions to be immoral themselves, and (b) whether they think immoral thoughts are likely to lead to action. These results built on previous research, showing that Jews and Protestants do not differ in the extent to which they view certain thoughts as moral or immoral but that Protestants more strongly believe that thinking about an immoral act is as bad as actually doing it. The author concluded that Protestants are therefore likely to use thoughts as a basis for negative moral judgement.

Similar research was conducted by Siev and Cohen (2007) who looked at differences in thought-action fusion between Christians and Jews using an online survey. Results showed that the Christians scored higher on the TAF-moral subscale than Jews. Additionally, in the Christian group, religiosity was only found to be associated with TAF, and not OCD cognitions and symptoms. The authors suggest that TAF-moral is only a marker of psychopathology when the beliefs are not culturally normative; however when they are present alongside religious doctrine, they may not be indicative of pathology.

The fact that TAF has been found to play a part in the development and maintenance of a number of psychological disorders, and that TAF has repeatedly been found to correlate with religiosity, may lead one to assume that religious people are therefore at higher risk of pathology. However, the evidence for such a relationship indicates a much more complex relationship. Interesting research by Siev, Chambless and Huppert (2010) evaluated TAF-moral alongside religious affiliation and OCD symptoms among undergraduate students. The findings of this research showed Christians endorsed higher levels of TAF-moral than Jews. Results also showed that among Christians, TAF-moral was related to religiosity, but not OCD symptoms. In contrast, for the Jewish participants, TAF-moral was related to OCD symptoms, but not religiosity.

Berman, Abramowitz, Pardue and Wheaton (2010) investigated the association between religion and TAF using an in-vivo paradigm. Protestant Christian and Atheist undergraduate students completed an exercise that involved writing the name of a close, loved relative into both negative likelihood thoughts ("I hope is in a car accident today"), and negative moral thoughts involving incest ("I hope I 
have sex with "). After spending time writing down the thought and visualising its occurrence, they completed measures of anxiety, likelihood of the event happening, and the moral wrongness of thinking and writing the thought. In terms of their affective responses, the groups reported equally high levels of anxiety experienced due to thinking about the events. The religious participants, however, rated the events as more likely to occur, and that thinking and writing these thoughts were more morally wrong, than did the Atheist participants. Even though the religious groups showed higher levels of induced TAF, this was not related to higher anxiety levels. The authors suggested that the religious participants were able to use beliefbased anxiety reduction strategies, such as praying for forgiveness.

\subsubsection{Religious Affiliation vs. Religiosity}

A lot of research in this particular area has included a measure of religiosity. Religiosity can be described as "society-based beliefs and practices relating to God or a higher power commonly associated with a church or organised group" (Egbert, Mickley \& Coeling, 2009; p.8). There are over 100 scales measuring religiosity and many of these differentiate between extrinsic and intrinsic orientations (Egbert et al., 2009). One of the more commonly used measures is the Duke Religion Index (DUREL; Koenig, Meador \& Parkerson, 1997), developed for use in health and psychiatric fields. This scale includes five items measuring external factors (e.g. "How often do you attend church of other religious meetings?"), and five measuring internal (e.g. "In my life, I experience the presence of the Divine"). Because these types of religiosity scales measure religiosity in general, it is difficult to attribute any observed differences to a particular doctrine, or to see where these differences may originate. The current study uses religious affiliation - either Protestant Christian or Atheist. This is dichotomous, as one cannot believe or disbelieve in God more than someone else. Religious affiliation means that the individual has self-identified with a particular religion, with the assumption being made that this reflects an internal decision to live according to that belief (whether Atheist or Christian).

\section{$5.2 \quad$ Study 4}

The aims of Study 4 were to compare TAF and inflated responsibility in Protestant Christians and Atheist/Agnostics, and to investigate the moderating role of religious affiliation on the relationships between TAF-moral and symptoms associated with OC and depression. Previous research has repeatedly found TAF-moral to be significantly associated with symptoms of depression. For example, Abramowitz, 
Whiteside, Lynam and Kalsy (2003) examined TAF in relation to anxiety and depression, and whether negative affect mediates the relationship between TAF and OCD. The participants were made up of clinical and non-clinical groups. Results showed that higher scores on the TAF scale were related to more severe depression and anxiety. More specifically, TAF-likelihood was related to anxiety, while TAFmoral was more related to depression. The authors propose that TAF-moral reflects types of cognitive distortions seen in depression such as self-blaming for external events. The association between TAF-moral and depressive symptoms was found for both the clinical and non-clinical groups.

While most of the previous research on TAF with religious groups has been conducted comparing Jews and Protestants/Catholics, Study 4 will compare Protestant Christians with Atheists. It is anticipated that the results will add to existing research that religious groups show higher levels of TAF. The study will extend the work of Siev, Chambless and Huppert (2010) on the moderating role of religiosity on TAFmoral and OC symptoms. It will extend and improve on this research by including a comparison group (Atheists), and being the first study to include a measure of depression. Research by Abramowitz et al. (2003) showed that TAF-moral is more directly related to depression. Research on religiosity shows more links with TAFmoral than TAF-likelihood (e.g. Rassin \& Koster, 2003). It is speculated that TAFlikelihood is more akin to tempting fate and superstition which goes against the more Calvinist doctrine which focuses more on Divine predeterminism (Rassin \& Koster). Predeterminism refers to the idea that events are determined in advance, and in Christianity this means that all future events are already decided by God (McKewan, 2009).

\subsubsection{Hypotheses}

Based on previous research, it was hypothesised that (a) participants with Protestant religious beliefs would demonstrate significantly higher levels of TAFmoral than Atheists; and (b) that TAF-moral would be significantly associated with OC symptoms and depression in the Atheist group, but not for Christian participants; i.e. that religiosity would moderate the relationship between TAF-moral and OC symptoms/depression. A measure of inflated responsibility was included to see whether there were group differences on this measure similar to TAF. As religious groups often show elevated levels of TAF, a measure of thought suppression was also 
included to see whether there was also an increase in attempts to avoid unwanted thoughts.

\subsection{Method}

\subsubsection{Participants}

Participants were 100 Protestant Christians, and 100 Atheists, who completed an anonymous online survey. Christian participants were recruited from local protestant (Baptist and Non-Denominational) churches and ranged from 16 to 80 years of age $(M=31.51, S D=12.90)$, with 34 males and 48 females (18 did not complete this question). Regular congregation members known to the Pastors were sent emails with information about the research and a link to the online survey. Atheist participants were recruited using a snowballing effect through a social networking site, and through a national Atheist society; they ranged from 18 to 80 years of age $(\mathrm{M}=35.42, \mathrm{SD}=16.67)$, with 50 males and 50 females.

\subsubsection{Measures}

The Obsessive Compulsive Inventory - Revised (OCI-R; Foa et al., 2002), the Responsibility Attitude Scale (RAS; Salkovskis et al., 2000), the Thought-Action Fusion Scale-Revised (TAFS-R; Shafran et al., 1996), the White Bear Suppression Inventory (WBSI; Wegner \& Zanakos, 1994), and the Self-Rating Depression Scale (Zung, 1965) were used in this study and are described in detail in Study 1 (Chapter 2).

\subsubsection{Procedure}

The questionnaire data were collected via an anonymous online survey using Qualtrics software (Qualtrics, 2013). For the Christian sample, emails were sent out from Church leaders inviting their congregations to participate. Atheist participants were recruited through Facebook, using a snowballing effect. Additionally, a national Atheist/Rationalist society emailed their members to participate, making up the Atheist group. Participation took approximately 15-20 minutes. The Victoria University Human Ethics Committee gave approval for this study prior to its beginning. The information, consent and debriefing information for this study is included in Appendix D. 


\section{$5.4 \quad$ Results}

\subsubsection{Descriptive Analyses}

The means, standard deviations and reliability coefficients for all variables are shown in Table 5.1. As can be seen, all scales showed good to excellent reliability (Cronbach's alphas).

Table 5.1

Mean scores (and standard deviations) and reliability analyses

\begin{tabular}{|c|c|c|c|c|}
\hline & $\begin{array}{l}\text { Atheist } \\
\mathrm{N}=100\end{array}$ & $\begin{array}{l}\text { Protestant } \\
\text { Christian } \\
\mathrm{N}=100\end{array}$ & $\begin{array}{c}\text { Total } \\
\mathrm{N}=200\end{array}$ & $\alpha$ \\
\hline TAF - total & $26.81(10.99)$ & $44.10(13.55)$ & $34.55(14.91)$ & 0.94 \\
\hline TAF - moral & 17.69 (7.27) & $35.56(12.41)$ & $25.86(13.38)$ & 0.96 \\
\hline TAF - likelihood & $8.94(4.37)$ & $8.52(3.33)$ & $8.68(3.99)$ & 0.93 \\
\hline - (others) & $4.83(2.51)$ & 4.54 (1.69) & $4.60(2.13)$ & 0.96 \\
\hline - (self) & $4.11(2.20)$ & $3.98(2.02)$ & $4.07(2.18)$ & 0.89 \\
\hline RAS & $57.44(27.23)$ & $64.32(25.91)$ & $60.63(26.77)$ & 0.93 \\
\hline SRDS & $35.00(9.30)$ & $37.6(10.04)$ & 36.08 (9.67) & 0.87 \\
\hline OCI-R & 10.77 (8.97) & $12.14(7.83)$ & $11.43(8.45)$ & 0.86 \\
\hline WBSI & $2.23(.88)$ & $2.67(.87)$ & $2.44(.90)$ & 0.93 \\
\hline
\end{tabular}

Note: $\alpha$ : Cronbach's alpha.

TAF-R: Thought-Action Fusion Scale - Revised with subscales (Shafran et al., 1996); RAS: Responsibility Attitude Scale (Salkovskis et al., 2000); SRDS: Self-Rating Depression Scale (Zung, 1965); OCI-R: Obsessive Compulsive Inventory - Revised (Foa et al., 2002); WBSI: White Bear Suppression Inventory (Wegner \& Zanakos, 1994).

Pearson Correlations are shown in Table 5.2 for both the Christian and Atheist participant groups. In the Christian sample, TAF-moral correlated with OC Symptoms, but not with Depression. In the Atheist sample, TAF-moral significantly correlated with both OC symptoms and depression. Thought suppression significantly correlated with OC symptoms, TAF-moral and Depression in both samples. 
Table 5.2

Pearson correlations

\begin{tabular}{|c|c|c|c|c|c|c|}
\hline & \multicolumn{3}{|c|}{ Protestant Christians $(\mathrm{N}=100)$} & \multicolumn{3}{|c|}{ Atheist/Agnostic $(\mathrm{N}=100)$} \\
\hline & 1 & 2 & 3 & 1 & 2 & 3 \\
\hline (1) TAF-Moral & 1 & & & 1 & & \\
\hline (2) OCI-R & $.243 *$ & 1 & & $.498 * *$ & 1 & \\
\hline (3) SRDS & .166 & $.575^{* *}$ & 1 & $.334 * *$ & $.532 * *$ & 1 \\
\hline (4) WBSI & $.354 * *$ & $.656^{* *}$ & $.549 * *$ & $.308 * *$ & $.576^{* *}$ & $.549 * *$ \\
\hline
\end{tabular}

Note: $* p<.01, * * p<.001$.

TAF-Moral: subscale from TAF-R: Thought-Action Fusion Scale - Revised (Shafran et al., 1996); OCI-R: Obsessive Compulsive Inventory - Revised (Foa et al., 2002); WBSI: White Bear Suppression Inventory (Wegner \& Zanakos, 1994); SRDS: SelfRating Depression Scale (Zung, 1965).

\subsubsection{Group Differences}

As can be seen in Figure 5.1, Protestant Christians demonstrated higher overall TAF scores than Atheist participants $(t[198]=9.48, p<.001)$. However, when this is divided into TAF-moral and TAF-likelihood, only TAF-moral scores are significantly different $(t[198]=11.56, p<.001)$, between the groups, with the Christian group being higher on this measure. The two groups also differed significantly on thought suppression, with Protestant Christians scoring higher on this scale than the Atheist group $(t[198]=3.47, p<.001)$. There were no significant differences between the two groups on inflated responsibility, depression, OC symptoms and TAFlikelihood. 


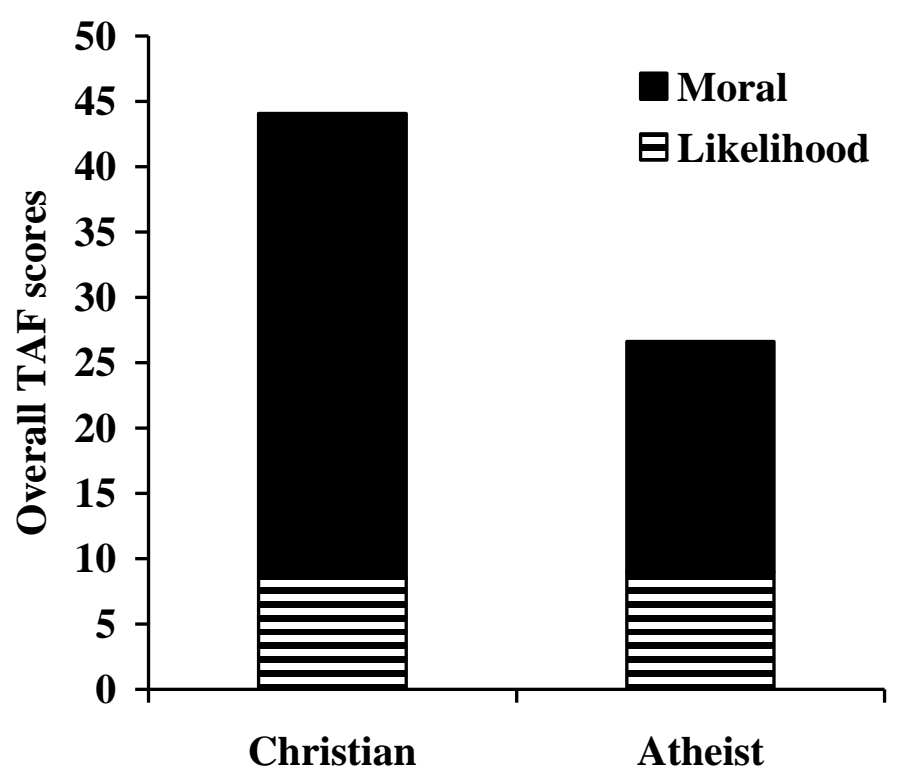

Figure 5.1 Differences between Christians and Atheists on overall TAF, TAF-moral and TAF-likelihood. TAF total and TAF-moral differences are significant.

\subsubsection{Moderation}

The moderation analyses were performed using Modgraph (Jose, 2008), which is based on the guidelines by Aiken and West (1991). Before analyses were performed, TAF-moral as an independent variable was centred to reduce multicollinearity. Then simple regression lines for moderated variables were plotted for significant interaction effects.

\subsubsection{OC Symptoms}

As can be seen in Figure 5.2, religiosity moderates the relationship from TAFmoral to OC symptoms. That is, an increase in TAF-moral scores predicted an increase in OC symptom severity for the Atheist participants. Comparison of this slope showed a significant difference from zero $(\beta=0.16, t[196]=2.23, p<.05)$. In comparison, OC symptom severity decreased with an increase in TAF-Moral for the Christian participants. Comparison of the slope against zero, however, revealed that the simple slope for the Christian participant group was not significant $(\beta=-0.26$, $t[196]=-1.48, p=0.14)$. 


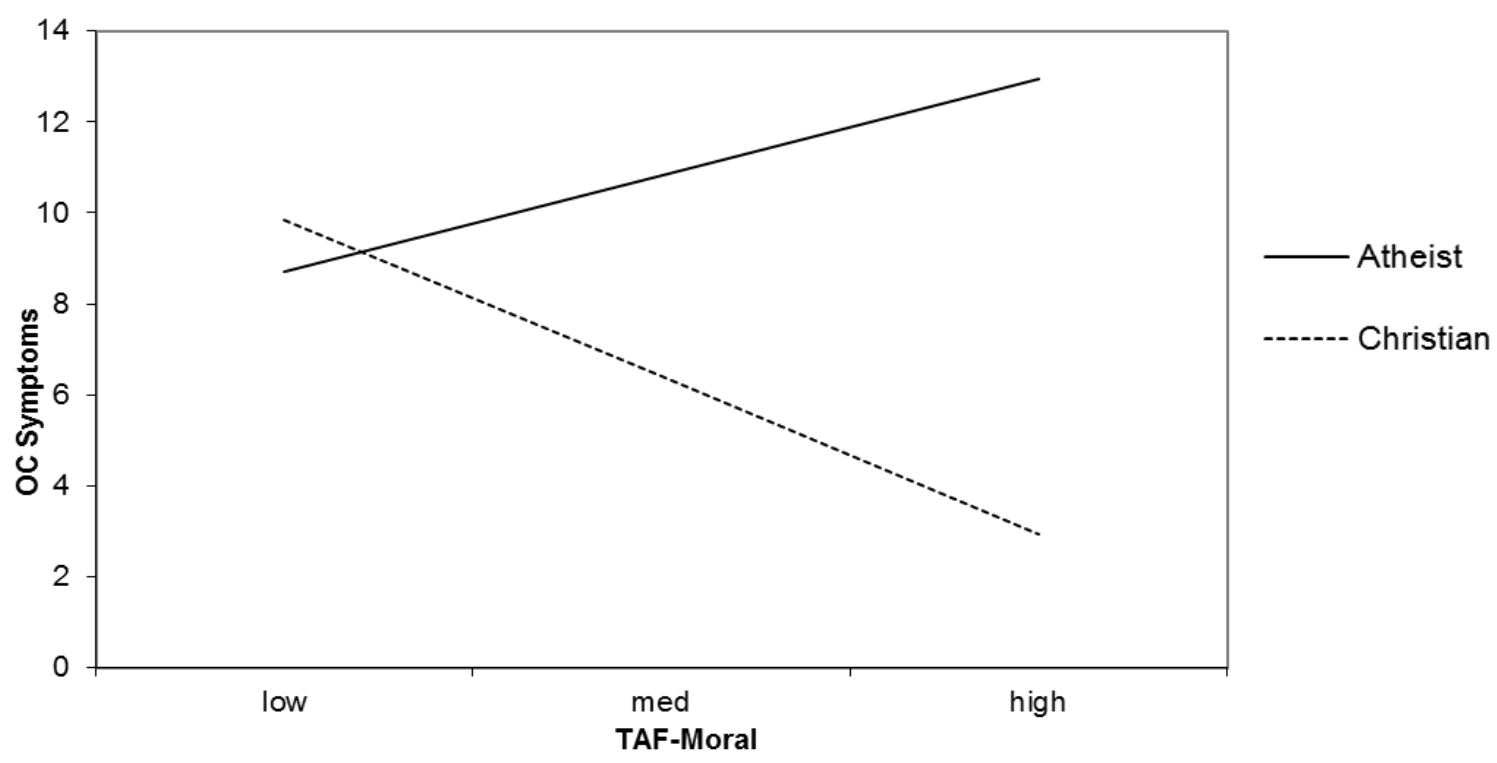

Figure 5.2 Religiosity as a moderator for TAF-Moral on OC symptoms.

\subsubsection{Depression}

Figure 5.2 shows the moderating role of religiosity from TAF-moral to

Depression scores. Even though the pattern of results resembles the pattern observed for OC symptoms, neither of the slopes differed significantly from zero (Christian sample: $\beta=-0.04, t[196]=-0.19, p=0.85$; Atheist sample: $\beta=0.13, t[196]=1.35, p=0.18$ ).

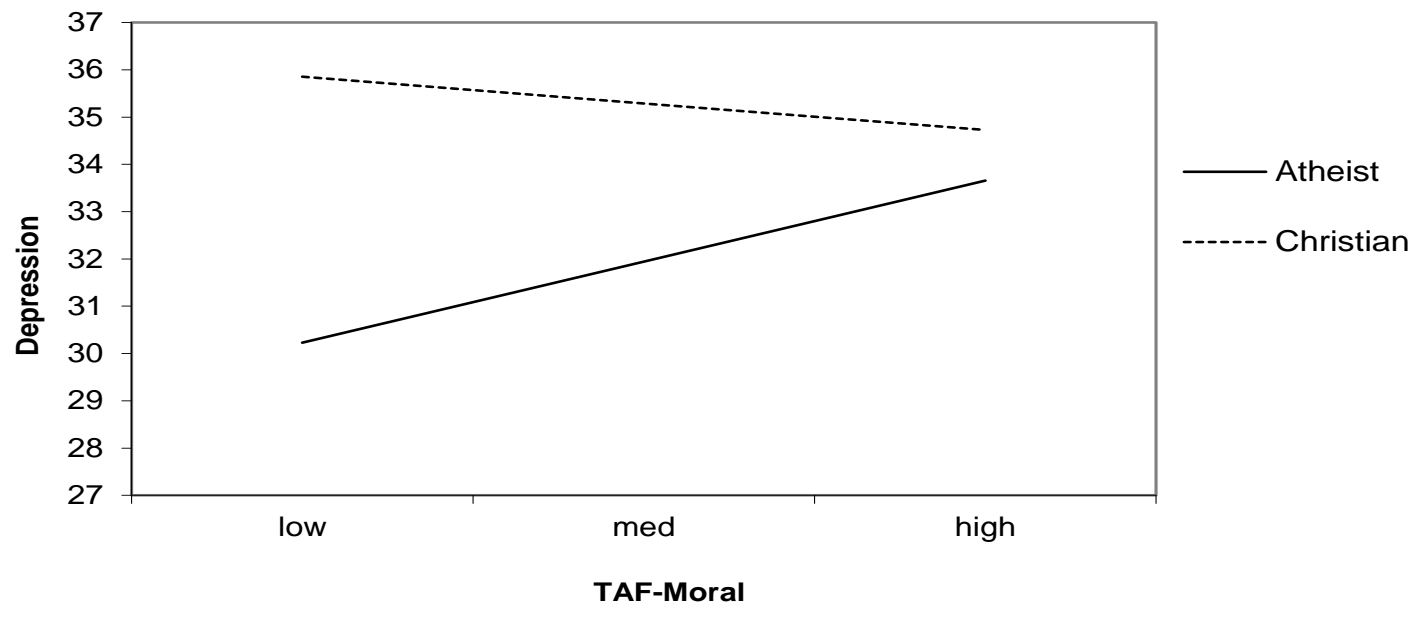

Figure 5.3 Religiosity as a moderator for TAF-Moral on Depression 


\subsection{Study 4 Discussion}

The goal of Study 4 was to examine the moderating role of religiosity in the relationship between responsibility beliefs and the occurrence of OC and depression symptoms. The current study examined religiosity as a dichotomous "religious" (i.e., Protestant Christian) vs. "non-religious" (i.e., Atheist) factor. Significant differences were found between the two groups on TAF-moral and thought suppression, with the Christians scoring higher on both measures. This difference was specific to TAFmoral, and did not generalise to TAF-likelihood or inflated responsibility. There were no significant differences between the two groups on depression or OC symptoms. Moderation analyses revealed that for the Christians, higher levels of TAF-moral was not associated with higher levels of OC symptoms, whereas with the Atheist group, higher TAF-moral scores were related to higher OC scores. It was expected that similar results would be found with depression. Results, however, showed that high TAF-moral scores were not related to higher depression scores for either group.

In this study, the Atheist group represented those individuals who do not endorse any type of religious belief. The obtained results were comparable to that of Siev et al. (2010) regarding OC symptoms in their Jewish sample. Within the Atheist sample, high scores on TAF-moral were significantly associated with higher scores on OC symptomatology. Although the same pattern was hypothesised for scores on depression, this was not found.

The finding that, for those holding Christian beliefs, TAF-moral was not associated with OC symptoms supported the hypothesis. When this is broken down further, it indicates that TAF-moral thoughts do not necessarily lead to feelings of anxiety and/or the desire to neutralise them through compulsive behaviours, as is measured by the OCI-R (Foa et al., 2002). Arguably there may be something inherently different about the way that Christians process such thoughts compared to non-Christians. This can be related to Rachman's (1997) Misinterpretation of Significance Theory which proposes that for a thought to become pathological, the individual misinterprets it as being personally meaningful and indicates something significant about their character, which may lead to negative consequences. Clark (2004) proposed that those who show high levels of the TAF bias are vulnerable to making such misinterpretations. In terms of the Christian group, there appears to be at least two options, either they are not making any such misinterpretations, or they are, and this is being offset by another factor. Given the teachings of the Bible around 
thoughts being judged, and being morally equivalent to actions, the latter appears more likely. It may be that the added teachings of the unconditional mercy and forgiveness of God in Christian theology works to neutralise any anxiety caused by the thoughts.

\subsubsection{Christian Theology}

For the results to show twice the levels of TAF-moral in the Christian group compared to the Atheist group, indicates there is something within Christian teaching that links thoughts to actions, at least in terms of morality. Christianity is a belief system which places great importance on one's thoughts and intentions (Cohen \& Rozin, 2001). Evidence for this can be seen throughout the Bible, for example "The Lord knows the thoughts of man” (Psalm 94:11; New International Version); “... you perceive my thoughts from afar" (Psalm 139:2); "For the word of God is living and active... it judges the thoughts and attitudes of the heart" (Hebrews 4:12), and of course "You have heard that it was said, "Do not commit adultery." But now I tell you: Anyone who looks at a woman and wants to possess her is guilty of committing adultery with her in his heart" (Matthew 5: 27-28). These passages describe that God knows peoples' thoughts, and that when judged by God, people are represented by their thoughts. Furthermore, merely the act of thinking about a negative action is considered sinful (often referred to as 'sin by thought'). It would logically follow, then, that those who choose to live by a certain interpretation of these words would be more likely to endorse TAF-moral statements.

This could also help to explain the significantly higher levels of thought suppression. As discussed in Chapter 2, there are concerns with the validity of the White Bear Suppression Inventory (WBSI; Wegner \& Zanakos, 1994) and its ability to measure actual thought suppression, as opposed to cognitive intrusions. Either way, a high score on this scale indicates that the individual experiences thoughts they do not want, and that they may try to suppress or avoid. This elevated rate of thought suppression in the Christian sample may not necessarily be related to TAF-moral cognitions, but may be more generalised. That is, it may be an indicator that those with Christian beliefs are more mindful of their thoughts, and engage in more selfmonitoring to avoid what they consider to be immoral or sinful thoughts, compared to non-Christians. Currently this conclusion is speculative and more research on this is needed. 


\subsubsection{Religious Affiliation}

The obtained results cannot be generalised to other religions, and crossreligion commonality should not be assumed. Even within Protestant Christianity, there are different denominations with variations in doctrine and practice. Rather than examining all existing religious groups, it may be possible to find patterns of thought along categories of religions; it may be that people of different religions make their moral judgements depending on their taught doctrine (Cohen, 2003). One way to distinguish between groups of religions is the degree to which they are Orthodox or Orthopractic. Orthodox religions emphasise internal beliefs and creed, whereas Orthopractic religions are more focused on law and community life (Cohen). In other words, Orthodox religions are more internally focussed, on thoughts and beliefs (e.g. Christianity), whereas orthopractic religions are more externally focused and emphasise the importance of behaviours and practices (e.g. Islam and Judaism). A similar classification system is whether religions are descent or assent in terms of how membership is defined. In descent religions, members are decided through birth (e.g. Judaism and Hinduism), whereas membership to assent religions requires the personal decision to adopt a particular belief structure (e.g. Christianity and Buddhism). Cohen (2003) speculates that assent religions would be more moralising of thoughts, i.e. that they would score higher on TAF-moral, as they require personal, internal beliefs. The same may be said for religions that are more orthodox; that they centre around belief structures and thoughts may mean they are more likely to endorse TAF-moral, as opposed to the more orthopractic religions.

Most previous research comparing TAF and morality between religious groups has been with Christian and Jews (e.g. Cohen \& Rankin, 2004; Siev, Chambless \& Huppert, 2010). Christianity is an example of a more orthodox, assent religion, while Judaism is more orthopractic and a religion of descent. Christianity emphasises the notion that one's eternal life depends on commitment to a belief, and teaches few restrictions and behaviours. However, Judaism teaches behavioural adherence to daily routines and law, and places little importance on mental states. Crucial differences in doctrine, theology and practise of religion make it so important to not make assumptions in terms of TAF-moral and related cognitions. While many studies vaguely measure 'religiosity', this can lead readers to unintentionally relate this to a traditional Christian perspective, while in fact there is so much variance across different religions, sects and denominations. 


\subsubsection{Clinical Implications}

The current research has important clinical implications; sensitivity around religious beliefs is needed when approaching individuals about their spiritual and moral beliefs, as well as their perceived moral weight of thoughts, desires and intentions. As concluded by Siev, Chambless and Huppert (2010), when TAF beliefs are culturally normative, as in religious teachings, they do not necessarily signal pathology, and can be an example of healthy religious beliefs. Clinicians and researchers need to consider the context of responsibility beliefs, before assuming that they represent a risk factor for psychological disorder. Clinicians in particular, should be aware that attempting to convince religious patients that their thoughts do not matter or are incorrect may be insensitive and unhelpful. Rather, Siev and Cohen (2007) recommend focussing on the interpretation and implications for self-worth, based on these thoughts. Additionally, they recommend directing attention more on educating clients that perfection is a goal demanded by OCD and not religion, rather than spending time on whether the thoughts matter.

\subsubsection{Limitations}

Both groups of participants were very aware that they had been selected for being either Christians or Atheists, which may have had an influence on the way they responded. For example, the Christian sample may have responded in a way that they believe 'good Christians' should. Similarly, the Atheists may have responded in strong opposition against the more religious items as they knew their views on this were of interest. The thought-action fusion scale used in this study has a number of items that directly mention God/Church which potentially would have drawn this out, with the two groups of participants responding at either ends of the scale. For example "Having a blasphemous thought is almost as sinful to me as a blasphemous action", and "When I think about making an obscene remark or gesture in church, it is almost as sinful as actually doing it". In future research, it would be interesting to either exclude these items, or to use modified items to take away the religious themes, and see whether this may account for the differences in overall TAF scores.

\subsubsection{Future Research}

TAF-moral is nearly always considered in terms of thoughts about doing negative, immoral things. Similarly, the Revised TAF Scale (TAFS-R; Shafran et al., 1996) only describes unwanted, negative cognitions. This makes sense, given the associations with anxiety and OC symptoms. It would be unlikely for individuals to 
seek help from a mental health professional because of positive, happy thoughts. As Shafran and Rachman (2004) describe, it is unlikely for those with OCD to believe that their positive thoughts will increase the likelihood of positive events. However, in terms of TAF-moral in relation to religious beliefs, it would be interesting to examine whether the same differences between Christians and Atheists could be found with positive thoughts. For example, does simply thinking about giving to charity make someone charitable, or does thinking about being a loving wife/husband make them a good spouse? For example, Cohen and Rankin (2004) found that Christians paid more attention to underlying motivations for virtuous actions; for example, selfish motives invalidated the moral quality of the action; this was not found for the Jewish sample. This is in line with Jewish views that "thoughts about immoral actions are natural and can be overcome, whereas thoughts about virtuous actions will be cultivated and acted on" (Cohen, 2003; p. 274).

\subsection{Chapter Summary}

The results of Study 4 showed that in the Christian sample, TAF-moral was not associated with OC symptoms or depression. This is compared to the Atheist group, where higher levels of TAF-moral were associated with higher scores on OC symptoms. TAF is often considered to be a marker of psychopathology, or a specific symptom of OCD. The results from Study 4 have shown that this is not always the case, and that TAF-moral beliefs in particular, are not necessarily maladaptive. This research has important implications for clinicians in considering the context of TAF beliefs before assuming they represent a risk factor for psychopathology. Attempting to correct these beliefs or convince someone that their thoughts are incorrect may be insensitive and damaging. As Siev and Cohen (2007) suggest, it may be more useful in a clinical setting, to help the individual reinterpret their thoughts in terms of the person's perceived self-worth, i.e. that their thoughts matter, but are not their fault. This research will also provide a better understanding on the links between TAF and religious affiliation (Protestant Christianity) in general, leading to more effective intervention and treatment techniques, while being respectful to individuals' beliefs. 


\section{CHAPTER SIX \\ 6. RESEARCH SUMMARY AND GENERAL DISCUSSION}

\subsection{Research Summary}

This body of research has been made up of four key studies on thought-action fusion and inflated responsibility. Study 1 was conducted to investigate the role of these beliefs in psychopathology alongside thought suppression. Studies 2A and 2B examined the additional influence of an individual's locus of control and how this effects responsibility beliefs. In Studies 3A and 3B, Salkovskis et al.'s (1999) pathways theory was empirically tested with both non-clinical and clinical participants, looking at the etiology of responsibility beliefs. Finally, Study 4 focussed on the relationship between responsibility beliefs and religiosity. The assessment methods included self-report questionnaires tapping into both TAF and inflated responsibility constructs.

\subsubsection{Study 1}

Study 1 used structural equation modelling to explore the relationships between both TAF and inflated responsibility beliefs and OC symptoms, depression and anxiety. The mediating role of thought suppression was also examined. It was hypothesised that both inflated responsibility and TAF would be related to symptoms of OCD, depression and anxiety, and that this relationship would be mediated by thought suppression. The results showed that while TAF was specific to OC symptoms, inflated responsibility was a more general construct and is also associated with depression and anxiety. For inflated responsibility, the best fitting structural model showed thought suppression to partially mediate between these responsibility beliefs and the three disorders. TAF-likelihood was directly linked to OC symptoms, depression and anxiety, while for TAF-moral this relationship was mediated by thought suppression. This research highlights important differences between TAF and inflated responsibility and how they are related to psychopathology. The findings that TAF-moral and inflated responsibility work alongside thought suppression have implications for treatment focusing on the underlying belief systems that may lead to the desire to suppress such thoughts, thereby potentially lessening symptoms of anxiety and depression, rather than the reaction of thought suppression which plays a more intermediary role. 


\subsubsection{Studies $2 A$ and $2 B$}

The aim of Studies 2A and 2B were to look at the function of control beliefs; namely having an external locus of control, and how this may work alongside responsibility beliefs in psychopathology. This was first investigated within a nonclinical sample (Study 2A), and then within a clinical group of anxiety patients (Study 2B). It was hypothesised that the interaction between responsibility beliefs and an external locus of control would predict OC symptoms. For the non-clinical sample, the results showed that the combination of having both an inflated responsibility and an external locus of control was predictive of OC symptoms. For the clinical sample, inflated responsibility and external locus of control were both individually predictive of higher scores on OC symptoms; however the interaction between these two variables was not significant. Study 2B also included a measure to TAF in order to see if there were similar findings for this type of responsibility belief. Results showed that only TAF-likelihood was related to external locus of control (and not TAF-moral). For overall TAF scores and TAF-likelihood, having an external locus of control fully mediated between these beliefs and higher OC symptoms. The combined results of both studies show how the two constructs of responsibility beliefs (both inflated and TAF) can work with an external locus of control to produce OC symptoms. It is proposed that it is the discrepancy between the level of responsibility one feels, and the level of control they have which is what leads to distress (Burger, 1992).

\subsubsection{Studies $3 A$ and $3 B$}

Studies 3A and 3B focused on the etiology of responsibility biases and empirically tested Salkovskis et al.'s (1999) theory of multiple pathways to inflated responsibility beliefs, with a newly designed measure. This was the first study to empirically test this theory within a general adult population, as well as with an adult clinical sample. It was hypothesised that these theoretical pathways would be predictive of inflated responsibility and TAF. For Study 3A with a non-clinical sample, results showed that the Rigid Rules and Actions pathways were significant predictors of inflated responsibility. This is consistent with the view that, growing up in an environment with strict rules and standards; and secondly, experiencing a catastrophic event where one believes they were responsible, are both related to later developing an inflated sense of responsibility. The same pathways were significant predictors in study $3 \mathrm{~B}$ with a clinical sample, as well as Overprotection. This pathway describes a childhood where the parent/s overprotected the individual in an 
environment of anxiety and worry. Study 3B also included a measure of TAF to see whether the pathways theory could predict these types of responsibility beliefs. Results showed that TAF-likelihood was predicted by the Actions pathway, and that TAF-moral was predicted by the Rigid Rules and Actions pathways. The overall results provided some support for Salkovskis et al.'s theory in not only predictive inflated responsibility, but also TAF beliefs. The most convincing results for both studies were found for the Actions pathway, which demonstrates the importance of how one processes their role (whether real or imagined) in causing a catastrophic event.

\subsubsection{Study 4}

The aim of Study 4 was to take a close look at the link between responsibility beliefs, religious affiliation and symptoms of OC and depression, and to examine the implications of this for treatment. This involved a comparison of Atheists and Protestant Christians. Based on previous research in this area, it was hypothesised that the Christian group would show higher levels of TAF-moral, and that TAF-moral would be associated with OC symptoms and depression in the Atheist group, but not for the Christian group. The results for this study showed that for the Christian sample, scores of TAF-moral were over twice as high as those of the Atheist group. In terms of the link to OC symptoms, for the Christian group, higher TAF-moral scores were not significantly related to having higher OC scores, while they were for the Atheist sample. For depression scores, higher TAF-moral scores were not significantly related for either of the two groups. These results have important implications for treatment, especially for those individuals with strong religious beliefs. It is important to consider and be respectful of the context of such beliefs before assuming they are representative of psychopathology. Additionally, attempts to correct such beliefs may be insensitive and offensive, as well as not being effective in reducing symptoms.

\subsection{The relationship between Thought-Action Fusion and Inflated Responsibility}

Across all studies in this research that included both constructs, the correlation between IR and TAF ranged from $r=.21$ to $r=.58$, indicating a weak to strong relationship. While TAF and inflated responsibility are significantly correlated, there are also important differences between the two. For example, Study 1 showed that TAF is specific to OC symptoms, while inflated responsibility seems to be a more general cognitive bias also evident in anxiety and depression. Overall results tend to 
support inflated responsibility being more general, while TAF tends to be specific to OC symptoms and religious affiliation. This supports research by Rassin et al. (1999) and Shafran et al. (1996), that the two belief types are closely related and theoretically connected, but also distinct. In Chapter 1, two options were proposed: either the two belief types shared some overlap, or TAF was a subset of inflated responsibility (refer to Figure 1.1). Based on the overall research results throughout the studies of this thesis, there is support for the first option, where there is some overlap and correlation, but each can occur without the presence of the other. Other researchers (e.g. Rachman, 1993; Rachman et al., 1995; Rassin, Merckelback, Muris and Spaan, 1999; Shafran et al., 1996) suggest that TAF leads to inflated responsibility, a suggestion that which would require longitudinal research to investigate.

\subsubsection{Misinterpretation of Significance Theory}

In addition to clarifying the relationship between TAF and inflated responsibility, it is also important to elucidate how this fits in with existing theory. Rachman's (1997; 1998) misinterpretation of significance theory describes how intrusive thoughts are misinterpreted in terms of personal significance, which leads to and maintains psychological distress (described in detail in Chapter 1). Clark (2004) proposes that responsibility biases such as TAF and inflated responsibility represent a vulnerability for making incorrect appraisals of intrusive thoughts. Therefore those individuals who endorse these beliefs are more likely to make misinterpretations of personal significance, thereby leading to (and maintaining) distress.

In reference to TAF beliefs in particular, Berle and Starcevic (2005) discuss whether these should be considered as beliefs or appraisals. They state that it remains unclear whether TAF is an appraisal specific to an intrusive thought, or that TAF beliefs represent a more enduring way of thinking. The authors tentatively conclude that TAF can be both; the individual can have TAF as an underlying trait-like characteristic, which is then used in specific situational appraisals. They suggest that "TAF may be considered as an appraisal when a specific preceding mental event (thought, impulse or image) is necessary for its occurrence and as a belief when it can be evoked in the absence of any particular mental event" (pg. 266).

\subsubsection{TAF-likelihood and TAF-moral}

For the correlation from the current research measuring each of the TAF constructs, the relationship between TAF-moral and TAF-likelihood was $r=.497$, indicating a strong relationship. This is comparable to the correlation value found by 
Shafran et al. (1996), of $r=.044$, and a similar strength to Rassin, Merckelbach, Muris and Schmidt (2001), who reported $r=.032$. The two constructs are clearly intertwined; Rachman and Shafran (1999) described that someone who believes their thoughts increases the likelihood of a negative event happening to someone else, is likely to also interpret these thoughts in terms of their morality for thinking something like this about another person.

However, throughout the studies of this thesis, evidence emerged that the two subtypes of TAF are very different. In each of the studies measuring TAF within this thesis, results have demonstrated important differences between likelihood and moral. For example in Study 1, TAF-moral works alongside thought suppression, while TAF-likelihood does not. In Study 2B, TAF-moral was not correlated with external locus of control while TAF-likelihood was. In Study 3B each subtype was associated with different theoretical pathways. Finally in Study 4, TAF-moral was associated with Protestant Christian beliefs, while TAF-likelihood was not. These findings suggest that it may not always be helpful or accurate to refer only to overall TAF scores. People who show high overall scores may actually only endorse one type of belief. This is shown in Study 4, where the Christian group showed significantly higher scores than the Atheists on overall TAF. However, when looking at the two subtypes, there was no significant difference in likelihood scores, and only a significant difference for moral scores. Therefore by looking only at the overall umbrella term of TAF, this may mask real underlying biases and therefore be unhelpful for identifying accurate information on these types of responsibility beliefs

Of course, the original study that described the psychometric properties of the TAF scale (Shafran, et al., 1996) identified a three-factor structure, dividing TAF into moral, likelihood-self and likelihood-other. More research on the similarities and differences between the two types of TAF-likelihood would be an interesting extension.

\subsection{The roles of Thought-Action Fusion and Inflated Responsibility in Psychopathology}

The research presented in this thesis provides evidence that TAF is specific to OCD. This is consistent with research showing this relationship (e.g. Coles et al., 2001; Rachman, 1993; Rachman et al., 1995; Shafran et al., 1996), however not other research showing TAF to be a cognitive bias in a wider range of disorders, for example anxiety (e.g. Muris et al., 2001; Abramowitz et al., 2003). The results from 
the current studies also reveal inflated responsibility to be an easily activated cognitive bias related to symptoms of OCD, depression and anxiety, supporting research that this type of belief is more general (Abramowitz et al.).

The results from the current body of research have provided many insights into the roles of both types of responsibility bias in psychopathology, namely anxiety, depression and OC symptoms. These symptoms are likely to be the result of a combination of variables including faulty responsibility beliefs, failed suppression attempts and external control beliefs. What can be taken from the current results is that inflated responsibility and TAF beliefs can play a maintaining role in particular psychological disorders, as these biases become imbedded in one's way of thinking and interpreting their place in the world. According to Rachman's (1997) misinterpretation of significance theory, distress and psychological symptoms persist over time, as the individual becomes stuck in a cycle where their thoughts are misinterpreted, leading to distress and therefore resistance, and attempts at thought suppression and neutralisation, rather than addressing the initial thought or image. Faulty belief patterns such as inflated responsibility and TAF set up the vulnerability to misinterpret thoughts according to these biases. Similarly, Salkovskis' $(1985 ; 1996)$ cognitive model proposes that symptoms are maintained by individuals' maladaptive responses to intrusive thoughts in terms of their personal responsibility, by directing their attentional efforts towards removing these thoughts, and therefore decreasing their feelings of inflated responsibility. Although these models have been developed in reference to OCD, it is suggested that the underlying principle of maintenance can be generalised to symptoms of anxiety and depression.

Results from the current research also highlighted how responsibility biases interact with control beliefs. In Studies $2 \mathrm{~A}$ and 2B an external locus of control was related to inflated responsibility and TAF which lead to higher scores on OC symptoms. For inflated responsibility this relationship was an interaction, whereas for TAF, external locus of control acted as a mediator. Locus of control may be considered similarly to responsibility biases in that it provides a way for individuals to interpret their thoughts. If they misinterpret these in a negative way, this can lead to attempts at suppressing thoughts, neutralising behaviours to reduce distress, and rumination. These reactions have been found to maintain disorders such as OCD, anxiety and depression (Rachman, 1997; 1998). 


\subsection{The Etiology of Thought-Acton Fusion and Inflated Responsibility}

As discussed by Salkovskis et al. (1999), the development of both inflated responsibility and TAF beliefs is likely to be the result of many different contributing factors over a period of time, which are subtle and difficult to identify. This may include parenting style, genetics, environmental and situational factors. The results of Studies 3A and 3B highlight the importance of critical incidents in this development. This refers to one part of the multiple pathways to inflated responsibility beliefs theory proposed by Salkovskis et al., and in the current research was also predictive of TAF beliefs (both moral and likelihood). It does not seem to make a difference whether the individual's role in the occurrence of the event is real or imagined. However, Salkovskis et al. note that the faulty responsibility beliefs that are generated from these types of events tend to be more specific, rather than generalising to all aspects of daily life. Studies 3A and 3B also show that growing up in an environment where there are extreme codes of conduct and behaviour are also conducive to developing responsibility biases (referring to the Rigid Rules pathway of Salkovskis et al.'s theory). In terms of the overall theory of pathways to responsibility beliefs, the current research provides empirical support for this idea, for both the non-clinical and clinical participant groups.

More research is needed on the factors involved in the development of biased responsibility beliefs. Some of these identified by Salkovskis et al. (1999) involve a history of being blamed, criticised and scapegoated, as well as situational increases in responsibility. Additionally, Berle and Starcevic (2005) suggest examining personality characteristics that may predispose an individual to developing faulty beliefs, such as neuroticism, harm avoidance and psychoticism.

\subsection{Clinical Implications}

The findings from the research throughout this thesis have some important implications for treatment around responsibility biases. Firstly, support was found for inflated responsibility being considered as a more general cognitive bias involved in anxiety and depression, rather than being a specific symptom or indicator of OCD. This suggests that it may be worthwhile to address such belief patterns in CBT with disorders other than OCD. By targeting underlying beliefs around responsibility, this may lead to a decrease in subsequent thought suppression and neutralisation attempts, and resultant symptoms of distress. Additionally, Studies 2A and 2B provide support for the idea that it is the discrepancy between high levels of personal responsibility 
beliefs and a low sense of control that leads to distress and psychological symptoms. Treatment programmes that incorporate developing more accurate attributions of personal control may also be helpful in decreasing the distress caused by responsibility biases.

TAF appraisals and inflated responsibility beliefs have been found to play a part in the development and maintenance of symptoms in a number of disorders and it is important to assess whether they are susceptible and amenable to change through therapy. Early promising research on this by Rassin et al. (2001) found that TAF beliefs decreased after successful cognitive-behavioural treatment of OCD and other anxiety disorders, although it was not specifically addressed. This suggests that perhaps the potency of therapy would be enhanced if there was some specific focus on TAF and responsibility beliefs. In a study with 72 undergraduate students, Zucker et al. (2002) found that providing participants who scored highly on measures of TAF with educational 'anti-TAF' scripts normalising intrusive thoughts, reduced anxiety and the urge to neutralise. Unfortunately there were no follow-up assessments, so it is unclear whether these effects persisted over time.

There have also been encouraging findings in terms of correcting inflated responsibility beliefs through therapy. For example, Ladouceur, Leger, Rheaume and Dube (1996) evaluated the efficacy of cognitive therapy targeting only inflated responsibility in four patients with OCD, using four key strategies: (a) targeting inflated responsibility (identifying situations in the patient's own life); (b) awareness of automatic thoughts; (c) correction of negative automatic thoughts; and (d) development of adequate perceptions of personal responsibility. All subjects reported a significant decrease in OC symptoms, as well as a decrease in perceived responsibility. These effects were maintained at follow ups (6 and 12 months) for three of the patients. In a more recent study, cognitive behavioural group therapy targeting inflated responsibility was found to significantly improve responsibility beliefs in 28 patients with OCD (Haraguchi, et al., 2011). Interestingly, results showed that while the treatment improved responsibility attitudes, the frequency of negative intrusive thoughts did not change. Research such as this is important in providing support for therapeutic techniques targeting inflated responsibility specifically. 


\subsection{Limitations and Directions for Future Research.}

Much of the research in this thesis is to do with causality, which has been inferred based on theory and past research. In order to get stronger evidence for these relationships, longitudinal research is needed. An additional limitation can be seen with the clinical sample used for Studies 2B and 3B. Although all participants had been formally diagnosed with anxiety disorders, they were all in the process of receiving treatment for these. This may have already begun to alter their underlying responsibility schemas; therefore if participants had completed this research before they began any treatment, their overall results may have been different.

As previously mentioned, longitudinal research is needed for more solid evidence around causality. These pathways have been inferred throughout the current research, based mainly on previous research. An example of this type of causality is from Study 4; an interesting question for future research is whether it is the religious teachings that lead to an endorsement of TAF-moral beliefs, or whether pre-existing TAF-moral beliefs lead to an interest in religiosity. In terms of religiosity itself, it would be worthwhile for clinical treatments to see whether there are other religious or cultural groups for which particular types of responsibility bias are considered normal, and not associated with symptoms of, or a vulnerability for psychopathology.

An additional extension to the current research is going beyond anxiety and depression, and investigating the potential role of responsibility beliefs in other disorders such as eating and psychotic disorders. For example there has been some work on a variation of TSF: Thought-Shape Fusion in eating disorders (Shafran et al., 1999). In terms of psychosis, it would be interesting to look at the links between responsibility beliefs and grandiose delusions seen in psychosis and manic states; whether there was any history of inflated responsibility before the onset of delusions, which set up a vulnerability for this.

Perhaps the most logical direction for future research is extending the investigation between TAF and inflated responsibility to also include magical ideation/thinking. Previous research has implicated magical thinking repeatedly in the discussion of responsibility beliefs, with some researchers even suggesting that TAF is a subtype of magical thinking (Einstein \& Menzies, 2004a; 2004b). More research on the role of magical thinking alongside responsibility, control and psychopathological symptoms would be valuable. 


\subsection{Conclusions}

To conclude, the studies presented in this thesis have demonstrated novel, empirical evidence to illustrate the significant roles and both inflated responsibility and TAF in psychopathology. It has been shown that both types of responsibility biases play different, but important roles in the development and maintenance of psychological symptoms related to anxiety, depression and OCD. The results support the idea of inflated responsibility being a general cognitive bias, while TAF is more specific to OC symptoms, and not always being an indicator of psychopathology. Considerable differences were found between TAF-moral and TAF-likelihood, indicating that it may not be helpful to consider these only in terms of overall TAF scores. By targeting underlying inflated responsibility and TAF beliefs during treatment, this may result in fewer attempts at thought suppression, neutralisation, and therefore symptoms and feelings of distress. 


\section{References}

Abramowitz, J., Deacon, B., Woods, C. \& Tolin, D. (2004). Association between protestant religiosity and obsessive-compulsive symptoms and cognitions. Depression and Anxiety, 20. 70. doi:10.1002/da.20021

Abramowitz, J.S., Whiteside, S., Lynam, D. \& Kalsy, S. (2003). Is thought action Fusion specific to obsessive-compulsive disorder?: a mediating role of negative affect. Behaviour Research and Therapy, 41. 1069-1079. doi:10.1016/S0005-7967(02)00243-7

Aiken, L.S. \& West, S.G. (1991). Multiple regression: Testing and interpreting interactions. Newbury Park, CA: Sage Publications.

Alloy, L.B. \& Riskind, J.H. (2006). Cognitive vulnerability to emotional disorders. Routledge.

Altin, M. \& Gencoz, T. (2011). How does thought-action fusion relate to responsibility attitudes and thought suppression to aggravate the obsessivecompulsive symptoms? Behavioural and Cognitive Psychotherapy, 39. 99114.

Altin, M. \& Karanci, A.N. (2008). How does locus of control and inflated sense of responsibility relate to obsessive-compulsive symptoms in Turkish adolescents? Journal of Anxiety Disorders, 22. 1303-1315. doi:10.1016/j.janxdis.2008.01.010

Amir, N., Cashman, L. \& Foa, E.B. (1997). Strategies of thought control in obsessive compulsive disorder. Behaviour Research and Therapy, 35. 775-777. doi:10.1016/S0005-7967(97)00030-2

Amir, N., Freshman, M., Ramsey, B., Neary, E. \& Brigidi, B. (2001). Thought-action Fusion in individuals with OCD symptoms. Behaviour Research and Therapy, 39. 765-776. doi: 10.1016/S0005-7967(00)00056-5

Baron, R.M. \& Kenny, D.A. (1986). The moderator-mediator variable distinction in social psychological research: conceptual, strategic, and statistical considerations. Journal of Personality and Social Psychology, 5. 1173-1182. doi:10.1037/0022-3514.51.6.1173

Beck (1976). Cognitive Therapy and Emotional Disorders. New York: International University Press.

Beck, A.T. \& Steer, R.A. (1987). Beck Depression Inventory: Manual. San Antonio, TX: The Psychiatric Corporation. 
Beevers, C.G., Wenzlaff, R.M., Hayes, A.M. \& Scott, W.D. (1999). Depression and the ironic effects of thought suppression: therapeutic strategies for improving mental control. Clinical Psychology: Science and Practice, 62. 133-148. doi:10.1093/clipsy.6.2.133

Benassi, V.A., Sweeney, P.D. Dufour, C.L. (1988). Is there a relation between locus of control orientation and depression? Journal of Abnormal Psychology, 97. 357-367. doi:10.1037/0021-843X.97.3.357

Bentler, P.M. (1992). On the fit of models to covariances and methodology to the bulletin. Psychological Bulletin, 112. 400-404. doi:10.1037/00332909.112.3.400

Berle, D. \& Starcevic, V. (2005). Thought-action fusion: review of the literature and future directions. Clinical Psychologyy Review, 25. 263-284. doi:10.1016/j.cpr.2004.12.001

Berman, N.C., Abramowitz, J.S., Pardue, C.M. \& Wheaton, M.G. (2010). The relationship between religion and thought-action fusion : use of an in vivo paradigm. Behaviour Research and Therapy, 48. 670-674. doi:10.1016/j.brat.2010.03.021

Bernard, L.C. \& Krupat, E. (1994). Health Psychology: Biopsychosocial Factors in Health and Illness. New York: Harcourt Brace College Publishers.

Bleuler, E. (1934). Textbook of Psychiatry. New York: The Macmillan Company. Blumberg, S.J. (2000). The white bear suppression inventory: revisiting its factor structure. Personality and Individual Differences, 29. 943-950. doi:10.1016/S0191-8869(99)00245-7,

Burger, J.M. (1984). Desire for control, locus of control, and proneness to depression. Journal of Personality, 52. 71-89. doi :10.1111/j.1467-6494.1984.tb00551.x

Byrne, R. (2006). The Secret. Atria Books : Beyond Words Publishing.

Chamberlain, S.R., Blackwell, A.D., Fineberg, N.A., Robbins, T.W. \& Sahakian, B.J. (2005). The neuropsychology of obsessive compulsive disorder: the importance of failures in cognitive and behavioural inhibition as candidate endophenotypic markers. Neuroscience and Biobehavioral Reviews, 29. 399419. doi:10.1016/j.neubiorev.2004.11.006

Clark, D.A. (2004). Cognitive-Behavioral Therapy for OCD. New York: The Guilford Press. 
Clark, D.A., Purdon, C.L. \& Wang, A. (2003). The meta-cognitive beliefs questionnaire: development of a measure of obsessional beliefs. Behaviour Research and Therapy, 41. 655-669. doi:10.1016/S0005-7967(02)00032-3

Cohen, A.B. (2003). Religion, likelihood of action, and the morality of mentality. The International Journal for the Psychology of Religion, 13. 273-285. doi:10.1207/S15327582IJPR1304_4

Cohen, A.B. \& Rankin, A. (2004). Religion and the morality of positive mentality. Basic and Applied Social Psychology, 26. 45-57. doi:10.1207/s15324834basp2601_5

Cohen, A.B. \& Rozin, P. (2001). Religion and the morality of religion. Journal of Personality and Social Psychology, 81. 697-710. doi:10.1037/00223514.81.4.697

Coles, M.E., Mennin, D.S. \& Heimberg, R.G. (2001). Distinguishing obsessive features and worries: the role of thought-action fusion. Behaviour Research and Therapy, 39. 947-959. doi:10.1016/S0005-7967(00)00072-3

Coles, M.E. \& Schofield, C.A. (2008). Assessing the development of inflated responsibility beliefs: the pathways to inflated responsibility beliefs scale. Behavior Therapy, 39. 322-335. doi:10.1016/j.beth.2007.09.003

Egbert, N., Mickley, J. \& Coeling, H. (2009). A review and application of social scientific measures of religiosity and spirituality: assessing a missing component in health communication research. Health Communication, 16. 727. doi: 10,1207/S15327027HC1601_2

Einstein, D.A. \& Menzies, R.G. (2004a). The presence of magical thinking in obsessive compulsive disorder. Behaviour Research and Therapy, 42. 539549. doi:10.1016/S0005-7967(03)00160-8

Einstein, D.A. \& Menzies, R.G. (2004b). Magical thinking in obsessive-compulsive disorder, panic disorder and the general community. Behavioural and Cognitive Psychotherapy, 34. 351-357. doi: 10.1017/S1352465806002864

Foa, E.B., Huppert, J.D., Leiberg, S., Langner, R., Kichic, R., Hajcak, G. \& Salkovskis, P.M. (2002). The obsessive-compulsive inventory: development and validation of a short version. Psychological Assessment, 14. 485-496. doi:10.1037/1040-3590.14.4.485

Ganellen, R.J. \& Blaney, P.H. (1984). Stress, externality and depression. Journal of Personality, 52. 326-337. doi:10.1111/j.1467-6494.1984.tb00355.x 
Garner, D.M. \& Bemis, K.M. (1982). A cognitive-behavioural approach to anorexia nervosa. Cognitive Therapy and Research, 6. 123-150. doi:10.1007/BF01183887

Gwilliam, P., Wells, A. \& Cartwright-Hatton, S. (2004). Does meta-cognition or responsibility predict obsessive-compulsive symptoms: a test of the metacognitive model. Clinical Psychology and Psychotherapy, 11. 137-144. doi:10.1002/cpp.402

Haraguchi, T., Shimizu, E., Ogura, H., Fukami, G., Fujisaki, M. \& Iyo, M. (2011). Alterations of responsibility beliefs through cognitive-behavioural group therapy for obsessive-compulsive disorder. Behavioural and Cognitive Psychotherapy, 39. 481-486. doi: 10.1017/S1352465811000051

Hazlett-Stevens, H., Zucker, B.G. \& Craske, M.G. (2002). The relationship of thought-action fusion to pathological worry and generalized anxiety disorder. Behaviour Research and Therapy, 40. 1199-1204. doi:10.1016/S00057967(01)00138-3

Hoelter, D.R. (1983). The analysis of covariance structures: goodness-of-fit indices. Sociological Methods and Research, 11. 325-344. doi:10.1177/0049124183011003003

Hofmann, S.G. (2005). Perception of control over anxiety mediates the relation between catastrophic thinking and social anxiety in social phobia. Behaviour Research and Therapy, 43. 885-895. doi: 10.1016/j.brat.2004.07.002

Hoping, W. \& de Jong-Meyer, R. (2003). Differentiating unwanted intrusive thoughts from thought suppression: what does the White Bear Suppression Inventory measure? Personality and Individual Differences, 34. 1049-1055. doi:10.1016/S0191-8869(02)00089-2

Jose, P. E. (2003) MedGraph-I: A programme to graphically depict mediation among three variables: The internet version, version 2.0. Victoria University of Wellington, Wellington, New Zealand. Retrieved from http://www.victoria.ac.nz/staff/paul-jose-files/medgraph/medgraph.php.

Jose, P.E. (2008). ModGraph-I: A programme to compute cell means for the graphical display of moderational analyses: The internet version, Version 2.0. Victoria University of Wellington, Wellington, New Zealand. Retrieved from http://www.victoria.ac.nz/psyc/staff/paul-jose-files/modgraph/modgraph.php.

Jose, P.E. (2010). Structural Equation Modelling Workshop. School of Psychology. 
Victoria University of Wellington, New Zealand.

Kennedy, B.L., Lynch, A.V. \& Schwab, J.J. (1998). Assessment of locus of control in patients with anxiety and depressive disorders. Journal of Clinical Psychology, 54 (4). 509-515. doi:10.1002/(SICI)1097 4679(199806)54:4<509::AID-JCLP12>3.0.CO;2-J

Kline, R.B. (1998). Software programs for structural equation modeling: Amos, EQS, and LISREL. Journal of Psychoeducational Assessment, 16. 343-364. doi:10.1177/073428299801600408

Koenig, H.G., Meador, K. \& Parkerson, G. (1997). Religious index for psychiatric research: A 5-item measure for use in health outcomes studies [Letter to the Editor]. American Journal of Psychiatry, 154. 885-886.

Ladouceur, R., Leger, E., Rheaume, J. \& Dube, D. (1996). Correction of inflated responsibility in the treatment of obsessive-compulsive disorder. Behaviour Research and Therapy, 34. 767-774. doi: 10.1016/0005-7967(96)00042-3

Lawrence, P.J. \& Williams, T.I. (2011). Pathways to inflated responsibility beliefs in adolescent obsessive-compulsive disorder: a preliminary investigation. Behavioural and Cognitive Psychotherapy, 39. 229-234. doi:10.1017/S1352465810000810

Lee, H. J., Cougle, J.R. \& Telch, M.J. (2005). Thought-action fusion and its relationship to schizotypy and OCD symptoms. Behaviour Research and Therapy, 43. 29-41. doi:10.1016/j.brat.2003.11.002

Lefcourt, H.M. (1972). Recent developments in the study of locus of control. In B.A. Maher (Ed.), Progress in Experimental Research in Personality (Vol. 6). New York: Academic Press.

Levenson, H. (1973). Multidimensional locus of control in psychiatric patients. Journal of Consulting and Clinical Psychology, 41. 397-404. doi: $10.1037 / \mathrm{h} 0035357$

Lopatka, C. \& Rachman, S. (1995). Perceived responsibility and compulsive checking: an experimental analysis. Behaviour Research and Therapy, 33. 673-684. doi:10.1016/0005-7967(94)00089-3

Marino, T.L., Lunt, R.A. \& Negy, C. (2008). Thought-action fusion: a comprehensive analysis using structural equation modelling. Behaviour Research and Therapy, 46. 845-853. doi:10.1016/j.brat.2008.03.005 
McKewan, J. (2009). Predeterminism. In H. Birx (Ed.), Encyclopedia of time: Science, philosophy, theology, \& culture. (pp. 1035-1036). Thousand Oaks, CA: SAGE Publications, Inc. doi: 10.4135/9781412963961.n191

Moulding, R. \& Kyrios, M. (2006). Anxiety disorders and control related beliefs: the exemplar of Obsessive-Compulsive Disorder (OCD). Clinical Psychology Review, 26. 573-583. doi:10.1016/j.cpr.2006.01.009

Moulding, R. \& Kyrios, M. (2007). Desire for control, sense of control and obsessivecompulsive symptoms. Cognitive Therapy and Research, 31. 759-772. doi:10.1007/s10608-006-9086-х

Nolen-Hoeksema, S. (2000). The role of rumination in depressive disorders and mixed anxiety/depressive symptoms. Journal of Abnormal Psychology, 109. 504-511. doi:10.1037/0021-843X.109.3.504

Papageorgious, C. \& Siegle, G.J. (2003). Rumination and depression: advances in theory and research. Cognitive Therapy and Research, 27. 243-245. doi:10.1023/A:1023918331490

Parkes, K.R. (1984). Locus of control, cognitive appraisal, and coping in stressful episodes. Journal of Personality and Social Psychology, 46. 655-668. doi:10.1037/0022-3514.46.3.655

Purdon, C. \& Clark, D.A. (1993). Obsessive intrusive thoughts in nonclinical subjects. Part 1. Content and relation with depressive, anxious and obsessional symptoms. Behaviour Research and Therapy, 31, (8). 713-720. doi:10.1016/0005-7967(93)90001-B

Purdon, C. \& Clark, D.A. (2001). Suppression of obsession-like thoughts in nonclinical individuals: impact on thought frequency, appraisal and mood state. Behaviour Research and Therapy, 39. 1163-1181. doi:10.1016/S00057967(00)00092-9

Qualtrics. (2013). Qualtrics Research Suite. Copyright ( ) 2013. Provo, UT, USA. Rachman, S. (1993). Obsessions, responsibility, and guilt. Behaviour Research and Therapy, 31. 149-154. doi:10.1016/0005-7967(93)90066-4

Rachman, S. (1997). A cognitive theory of obsessions. Behaviour Research and Therapy, 35,(9). 793-802. doi:10.1016/S0005-7967(97)00040-5

Rachman, S. (1998). A cognitive theory of obsessions: elaborations. Behaviour Research and Therapy, 36. 385-401. doi:10.1016/S0005-7967(97)10041-9

Rachman, S. (2003). The Treatment of Obsession. Oxford University Press, 
Incorporated.

Rachman, S. \& de Silva, P. (1978). Abnormal and normal obsessions. Behaviour Research and Therapy, 16. 233-248. doi:10.1016/0005-7967(78)90022-0

Rachman, S. \& Hodgson, R. (1980). Obsessions and compulsions. Hillsdale, NJ: Prentice-Hall.

Rachman, S. \& Shafran, R. (1999). Cognitive distortions: Thought-action fusion. Clinical Psychology \& Psychotherapy, 6. 8-85. doi:10.1002/(SICI)10990879(199905)6:2<80::AID-CPP188>3.0.CO;2-C

Rachman, S., Shafran, R., Mitchell, D., Trant, J. \& Teachman, B. (1996). How to remain neutral: an experimental analysis of neutralization. Behaviour Research and Therapy, 34. 889-898. doi:10.1016/S0005-7967(96)00051-4

Rachman, S., Thordarson, D.S., Shafran, R. \& Woody, S.R. (1995). Perceived responsibility: structure and significance. Behaviour Research and Therapy, 33. 779-784. doi:10.1016/0005-7967(95)00016-Q

Rassin, E. (2001). The contribution of thought-action fusion and thought suppression in the development of obsession-like intrusions in normal participant. Behaviour Research and Therapy, 39. 1023-1032. doi:10.1016/S00057967(00)00075-9

Rassin, E. \& Koster, E. (2003). The correlation between thought-action fusion and religiosity in a normal sample. Behaviour Research and Therapy, 41. 361-368. doi:10.1016/S0005-7967(02)00096-7

Rassin, E., Merckelbach, H., Muris, O. \& Spaan, V. (1999). Thought-action fusion as a causal factor in the development of intrusions. Behaviour Research and Therapy, 37. 231-237. doi:10.1016/S0005-7967(98)00140-5

Rassin, E., Muris, P., Schmidt, H. \& Merckelbach, H. (2000). Relationships between thought-action fusion, thought suppression and obsessive-compulsive symptoms: a structural equation modeling approach. Behaviour Research and Therapy, 38. (889-897). doi:10.1016/S0005-7967(99)00104-7

Salkovskis, P.M. (1985). Obsessional-compulsive problems: A cognitive-behavioural analysis. Behaviour Research and Therapy, 25. 571-583. doi:10.1016/00057967(85)90105-6

Salkovskis, P.M. (1996). Frontiers of cognitive therapy. New York, NY, US: Guilford Press,554. 
Salkovskis, P.M. \& Kirk, J. (1989). Obsessional disorders. In K. Hawton, P.M.

Salkovskis, J. Kirk \& D.M. Clark (Eds.). Cognitive behaviour therapy for psychiatric problems: A practical guide. Oxford medical publications. (pp. 129-168): Oxford University Press, New York, NY, US.

Salkovskis, P., Shafran, R., Rachman, S., \& Freeston, M.H. (1999). Multiple pathways to inflated responsibility beliefs in obsessional problems: Possible origins and implications for therapy and research. Behaviour Research and Therapy, 37, 1055-1072. doi:10.1016/S0005-7967(99)00063-7

Salkovskis, P.M., Thorpe, S.J., Wahl, K., Wroe, A.L. \& Forrester, E. (2003). Neutralizing increases discomfort associated with obsessional thoughts: an experimental study with obsessional patients. Journal of Abnormal Psychology, 112. 709-715. doi: 10.1037/0021-843X.112.4.709

Salkovskis, P.M., Wroe, A. L., Gledhill, A., Morrison, N., Forrester, E., Richards, C., Reynolds, M. \& Thorpe, S. (2000). Responsibility attitudes and interpretations are characteristic of obsessive compulsive disorder. Behaviour Research and Therapy, 38. 347-372. doi:10.1016/S0005-7967(99)00071-6

Shafran, R. \& Rachman, S. (2004). Thought-action fusion: a review. Journal of Behavior Therapy and Experimental Psychiatry, 35. 87-107. doi:10.1016/j.jbtep.2004.04.002

Shafran, R. \& Robinson, P. (2004). Thought-shape fusion in eating disorders. British Journal of Clinical Psychology, 43. 399-408. doi:10.1348/0144665042389008

Shafran, R., Thordarson, D.S. \& Rachman, S. (1996). Thought-action fusion in obsessive compulsive disorder. Journal of Anxiety Disorders, 10, (5). 379-391. doi:10.1016/0887-6185(96)00018-7

Siev, J., Chambless, D.L. \& Huppert, J.D. (2010). Moral thought-action fusion and OCD symptoms: the moderating role of religious affiliation. Journal of Anxiety Disorders, 24. 309-312. doi:10.1016/j.janxdis.2010.01.002

Siev, J. \& Cohen, A.B. (2007). Is thought-action fusion related to religiosity? Differences between Christian and Jews. Behaviour Research and Therapy, 45. 829-837. doi:10.1016/j.brat.2006.05.001

Smari, J. \& Holmsteinsson, H.E (2001). Intrusive thoughts, responsibility attitudes, thought-action fusion, and chronic thought suppression in relation to obsessive-compulsive symptoms. Behavioural and Cognitive Psychotherapy, 29. 13-20. doi:10.1017/S1352465801001035 
Smari, J., Porsteinsdottir, A., Magnusdottir, L., Smari, U.J. \& Olason, D.P. (2010). Pathways to inflated responsibility beliefs, responsibility attitudes and obsessive-compulsive symptoms: factor structure and test of a mediational model. Behavioural and Cognitive Psychotherapy, 38. 535-544. doi:10.1017/S135246581000041X

Strickland, B.R. (1989). Internal/external control expectancies: from contingency to creativity. American Psychology, 44. 1-12. doi:10.1037/0003-066X.44.1.1

Wegner, D.M., Schneider, D.J., Carter III, S.R. \& White, T.L. (1987). Paradoxical effects of thought suppression. Journal of Personality and Social Psychology, 53. 5-13.

Wegner, D.M. \& Zanakos, S. (1994). Chronic thought suppression. Journal of Personality, 62. 615-640. doi:10.1111/j.1467-6494.1994.tb00311.x

Wenzlaff, R.M., Wegner, D.M. \& Klein, S.B. (1991). The role of thought suppression in the bonding of thought and mood. Journal of Personality and Social Psychology, 20. 500-508.

Wenzlaff, R.M., Wegner, D.M. \& Roper, D. (1988). Depression and mental control: the resurgence of unwanted negative thoughts. Journal of Personality and Social Psychology, 55. 882-892. doi:10.1037/0022-3514.55.6.882

Woolley, J.D. (1997). Thinking about fantasy: are children fundamentally different thinkers and believers from adults? Child Development, 68. 991-1011. doi:10.1111/j.1467-8624.1997.tb01975.x

Yorulmaz, O., Altin, M. \& Karanci, N. (2008). Further support for responsibility in different obsessive-compulsive symptoms in Turkish adolescents and young adults. Behavioural and Cognitive Psychotherapy, 36. 605-617. doi:10.1017/S1352465808004530

Yorulmaz, O., Yilmazl, A.E. \& Gencoz, T. (2004). Psychometric properties of the thought-action fusion scale in a Turkish sample. Behaviour Research and Therapy, 42. 1203-1214. doi:10.1016/j.brat.2003.08.005

Zebb, B.J. \& Moore. M.C. (2003). Superstitiousness and perceived anxiety control as predictors of psychological distress. Journal of Anxiety Disorders, 17. 115130. doi:10.1016/S0887-6185(02)00176-7

Zucker, B.G., Craske, M.G., Barrios, V. \& Holguin, M. (2002). Thought action fusion: can it be corrected? Behaviour Research and Therapy, 40. 653-664. doi:10.1016/S0005-7967(01)00054-7 
Zung, W.W. (1965). A self-rating depression scale. Archives of General Psychiatry, 12 63-70. doi:10.1001/archpsyc.1965.01720310065008

Zung, W.W.K. (1971). A rating instrument for anxiety disorders. Psychosomatics, 12. 371-379. doi:10.1016/S0033-3182(71)71479-0 


\section{Appendix A}

Study 1 Information and Debriefing Information

VICTORIA UNIVERSITY OF WELLINGTON

Te Whare Wananga o te Upoko o te Ika a Maui

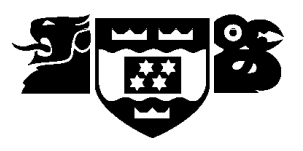

$\underline{\text { Information sheet }}$

$\mathrm{Hi}$, my name is Kirsty Fraser. I am undertaking this research to investigate aspects of everyday life, for example daily routines and mood. You'll also be asked questions about some sensitive topics (for example, your experience of disturbing, intrusive thoughts and your personal religious beliefs).

If you agree to take part in this study, you will be asked to fill out a questionnaire pack. Some of the questions you will be asked may seem very personal. Examples of such questions include those about your own religious beliefs and behaviours, as well as questions about your experience of disturbing, intrusive thoughts. Some items will ask for your perception of experiences such as: "I frequently get nasty thoughts and have difficulty in getting rid of them." Remember, you can withdraw from the study at any time.

As some of the questions in this study are sometimes used by other researchers to screen for individuals with depression, we will make contact with people whose scores indicate that they should seek support. Associate Professor John McDowall will write a letter to these people, asking them to visit him at an arranged time to discuss their scores, and support resources that are available to them. Though it is recommended, these people are under no obligation to make a subsequent appointment.

Data is confidential, and will be analysed collectively. The data will also be anonymously available to other competent professionals. There will be no way in which these other competent professionals will be able to identify you from the data.

If at any stage you wish to discontinue your participation in this research, you are welcome to withdraw from the study. If you choose to withdraw, this will not result in any penalty.

Thank you for your time.

Kirsty Fraser

Masters Student

kirsty.fraser@vuw.ac.nz
Associate Professor John McDowall

Senior Lecturer

john.mcdowall@vuw.ac.nz

I have read the information sheet and I give consent for my data to be used in this study.

Signature |

Student ID number | 
VICTORIA UNIVERSITY OF WELLINGTON

Te Whare Wananga o te Upoko o te Ika a Maui

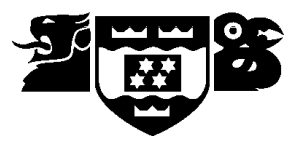

\section{Debriefing Sheet}

Thank you for participating in this research.

You have just completed several sets of questions used for a range of different purposes: to assess individuals with Obsessive Compulsive Disorder (OCD), depression, anxiety, religious beliefs, along with questions about your experience of disturbing, intrusive thoughts, responsibility beliefs and a measure of Thought-Action Fusion (TAF). TAF is an extremely common phenomenon whereby people have difficulty separating thoughts (in particular those that are negative and intrusive) from their corresponding behaviours. TAF was first introduced in the context of its occurrence in OCD; however there is a growing body of literature that suggests that TAF has implications in a wide variety of disorders, including depression and general anxiety.

The aim of this research was to investigate the relationships between TAF and a range of other variables, including Obsessive Compulsive Disorder, responsibility, depression, anxiety and religious beliefs. Relations between these variables and TAF have been found in previous research although in isolation. It is important to examine these relations and possible contributions simultaneously in order to elucidate how these constructs relate to one another. By investigating these relationships further, we hope to increase understanding and awareness of TAF and its potential role in a variety of clinical disturbances.

If questions in this study have raised any issues (either with regard to yourself or friends/family) that you wish to discuss further, please do not hesitate to contact Associate Professor John McDowall at the School of Psychology. Alternatively, please feel free to make use of the following services provided by the university:

$$
\begin{aligned}
& \text { Student Health Services } \\
& 4 \text { Wai-te-ata Rd } \\
& \text { Kelburn Campus } \\
& \text { (04) } 4635308
\end{aligned}
$$

Counselling Services

2 Wai-te-ata Rd

Kelburn Campus

(04) 4635310

Because the questionnaires in this study are used to screen for individuals with depression, we are obliged to make contact with people whose scores reveal that they should seek support. Associate Professor John McDowall will write a letter to these people, asking them to visit him at an arranged time to discuss the implications of their scores, and support resources that are available to them. Though it is recommended, these people are under no obligation to make a subsequent appointment.

If you wish to know the results of this study, or wish to discuss it further, please do not hesitate to contact Kirsty Fraser or Associate Professor John McDowall at the School of Psychology.

Thank you again for your time.

Kirsty Fraser

Masters Student

kirsty.fraser@vuw.ac.nz
Associate Professor John McDowall

Senior Lecturer

john.mcdowall@vuw.ac.nz 
Appendix B

Study 2A and 3A Information and Debriefing Information

VICTORIA UNIVERSITY OF WELLINGTON

Te Whare Wananga o te Upoko o te Ika a Maui

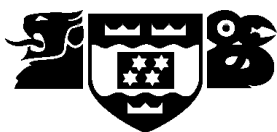

Information and Consent

This is an online survey investigating aspects about everyday life and anxiety. This research is being conducted by a $\mathrm{PhD}$ student at Victoria University of Wellington, New Zealand, and it has been approved by the Human Ethics Committee. This survey takes approximately 20-30 minutes to fill out. Please read through the consent form on the following page and click on "I agree" to proceed. You must be at least 16 years of age to complete the survey.

Thank you for your interest.

Kirsty Fraser \& Associate Professor John McDowall

Victoria University

Wellington

New Zealand

Please read this consent agreement carefully before you decide to participate in the study.

Purpose of the research study: This research aims to develop an understanding of the causes and factors related to anxiety.

Who is conducting the research?: Kirsty Fraser is a PhD student in the School of Psychology, and this research is supervised by Associate Professor John McDowall and Dr. Matt Crawford. This research has been approved by the Victoria University of Wellington ethics committee.

What is involved if you agree to participate?: If you agree to participate in this study, you will be asked to complete the online questionnaire. Some of the questions you will be asked may seem very personal. Examples of such questions include those about your childhood experiences and your own thoughts and behaviours. Some items will ask for your perception of experiences such as: "I frequently get nasty thoughts and have difficulty in getting rid of them." Remember, you can withdraw from the study at any time.

You will also be asked for some basic demographic information. We anticipate that your total involvement will take approximately 20-30 minutes.

Confidentiality: The questionnaire is totally anonymous so no individual's responses can or would be identified. We will keep the data collected here for at least five years after publication. You will never be identified in this research project or in any other 
presentation or publication. The information you provide will be coded by number only. In accordance with the requirements of some scientific journals and organisations, your coded data may be shared with other competent researchers. Your coded data may be used in other, related studies. A copy of the coded data will remain in the custody of Associate Professor John McDowall and will be kept in a locked cabinet in his office.

Voluntary participation: Your participation in the study is completely voluntary.

What happens to the information that you provide?: The data you provide may be used for one or more of the following purposes: The overall findings may be submitted for publication in a scientific journal, or presented at scientific conferences; The overall findings may form part of a PhD thesis, Masters thesis, or Honours research project that will be submitted for assessment.

Who to contact: Regardless of whether or not you complete the survey, if you have any general questions or comments about the research (excluding questions about the data you have provided), feel free to contact us.

Kirsty Fraser

School of Psychology

Victoria University of Wellington

Wellington, New Zealand

E-mail: kirsty.fraser@vuw.ac.nz

Associate Professor John McDowall

School of Psychology

Victoria University of Wellington

Wellington, New Zealand

E-mail: john.mcdowall@vuw.ac.nz

PLEASE FEEL FREE TO PRINT A COPY OF THIS PAGE FOR YOUR RECORDS.

Agreement. Do you agree to participate in the research study described above? 
VICTORIA UNIVERSITY OF WELLINGTON

Te Whare Wananga o te Upoko o te Ika a Maui

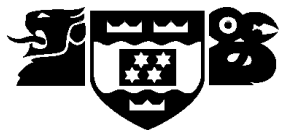

Debriefing Information

\section{Thank you for participating in this study}

Everyone has the occasional unwanted, and unwelcome, thought. It may involve behaviour that one may consider disturbing and very much out of character (e.g., imagining pushing someone in front of a bus), or a negative thought about possible future events (e.g., dying in a house fire). Most people are able to take these thoughts, dismiss them as strange, not act upon them, move on, and not think about them again. For others, however, these thoughts keep coming back again and again. For some people, these recurring unwanted thoughts can be very bothersome, for others, not so much.

The study that you just completed, examines early childhood experiences and the relationship between these experiences and adult mental health. Specifically, we are interested in how early childhood experiences relate to an inflated sense of responsibility (e.g., feeling that things are your responsibility and yours alone) and how this might be related to how well people are able to control unwanted thoughts in their daily lives.

By examining these questions, we can get a better understanding of how these may be related to the development of certain mental health issues which could provide invaluable information in how these might be treated for those who do have such a diagnosis.

If you have any concerns about your own well-being as a result of some of your responses to items within this survey, there are a number of sources from which you could seek further advice, for example meeting with your GP or Student Health (contact details below) to discuss your concerns.

Student Health Services

4 Wai-te-ata Rd

Kelburn Campus

(04) 4635308
Counselling Services

2 Wai-te-ata Rd

Kelburn Campus

(04) 4635310

If you have further questions, please feel free to contact either

Kirsty Fraser (Kirsty.Fraser@vuw.ac.nz) or

Associate Professor John McDowall (John.McDowall@ vuw.ac.nz)

at the School of Psychology, Victoria University of Wellington. 
Appendix C

Study 2B and 3B Information and Debriefing Information

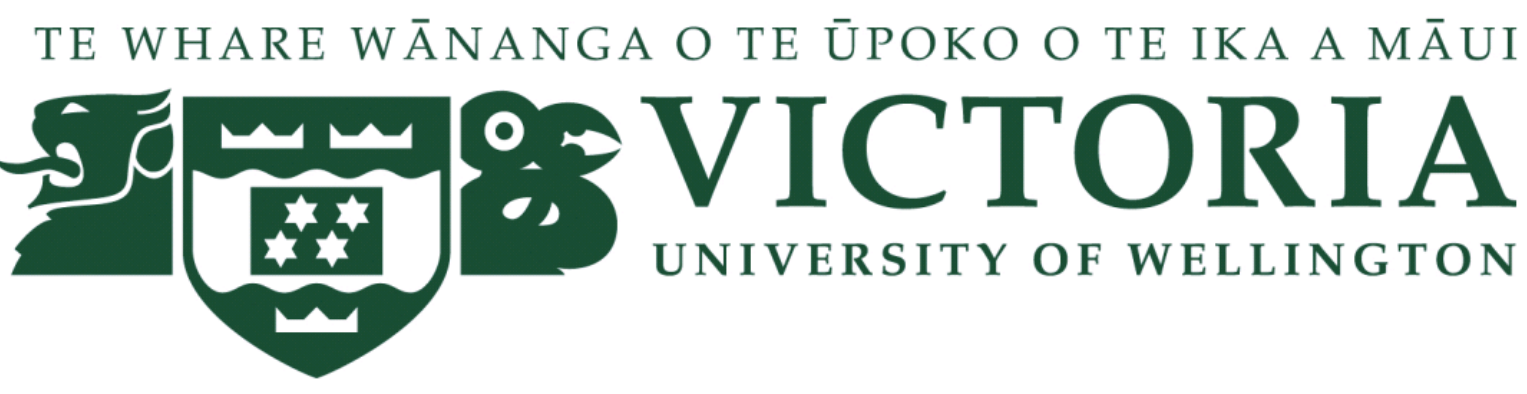

Information and Consent

This is a survey investigating aspects about everyday life and anxiety. This research is being conducted by a PhD student at Victoria University of Wellington, New Zealand, and it has been approved by the Human Ethics Committee. This survey takes on average 15-25 minutes to fill out. Please read through the consent form on the following page and click on "I agree" to proceed. You must be at least 16 years of age to complete the survey, and have been formally diagnosed as having Obsessive Compulsive Disorder.

Thank you for your interest.

Kirsty Fraser \& Associate Professor John McDowall

Victoria University

Wellington

New Zealand

Please read this consent agreement carefully before you decide to participate in the study.

Purpose of the research study: This research aims to develop an understanding of the causes and factors related to anxiety.

Who is conducting the research?: Kirsty Fraser is a $\mathrm{PhD}$ student in the School of Psychology, and this research is supervised by Associate Professor John McDowall and Dr. Matt Crawford. This research has been approved by the Victoria University of Wellington ethics committee.

What is involved if you agree to participate?: If you agree to participate in this study, you will be asked to complete the online questionnaire. Some of the questions you will be asked may seem very personal. Examples of such questions include those about your childhood experiences and your own thoughts and behaviours. Some items will ask for your perception of experiences such as: "I frequently get nasty thoughts and have difficulty in getting rid of them." Remember, you can withdraw from the study at any time. 
You will also be asked for some basic demographic information. We anticipate that your total involvement will take approximately 15-25 minutes.

Confidentiality: The questionnaire is totally anonymous so no individual's responses can or would be identified. We will keep the data collected here for at least five years after publication. You will never be identified in this research project or in any other presentation or publication. The information you provide will be coded by number only. In accordance with the requirements of some scientific journals and organisations, your coded data may be shared with other competent researchers. Your coded data may be used in other, related studies. A copy of the coded data will remain in the custody of Associate Professor John McDowall and will be kept in a locked cabinet in his office.

Voluntary participation: Your participation in the study is completely voluntary. It is entirely your choice if you would like to fill out the survey. Remember, if you feel uncomfortable at any time, it is ok to not finish.

What happens to the information that you provide?: The data you provide may be used for one or more of the following purposes: The overall findings may be submitted for publication in a scientific journal, or presented at scientific conferences; The overall findings may form part of a PhD thesis, Masters thesis, or Honours research project that will be submitted for assessment.

Who to contact: Regardless of whether or not you complete the survey, if you have any general questions or comments about the research, feel free to contact us. Please note we do not have access to your specific data.

Kirsty Fraser

School of Psychology

Victoria University of Wellington

Wellington, New Zealand

E-mail: kirsty.fraser@vuw.ac.nz

Associate Professor John McDowall

School of Psychology

Victoria University of Wellington

Wellington, New Zealand

E-mail: john.mcdowall@vuw.ac.nz

PLEASE FEEL FREE TO PRINT A COPY OF THIS PAGE FOR YOUR RECORDS.

Agreement. Do you agree to participate in the research study described above?

TO THE SURVEY.

YOU MUST BE OVER 16 YEARS OF AGE TO CONTINUE

I AGREE

$\ulcorner$ I DO NOT AGREE 


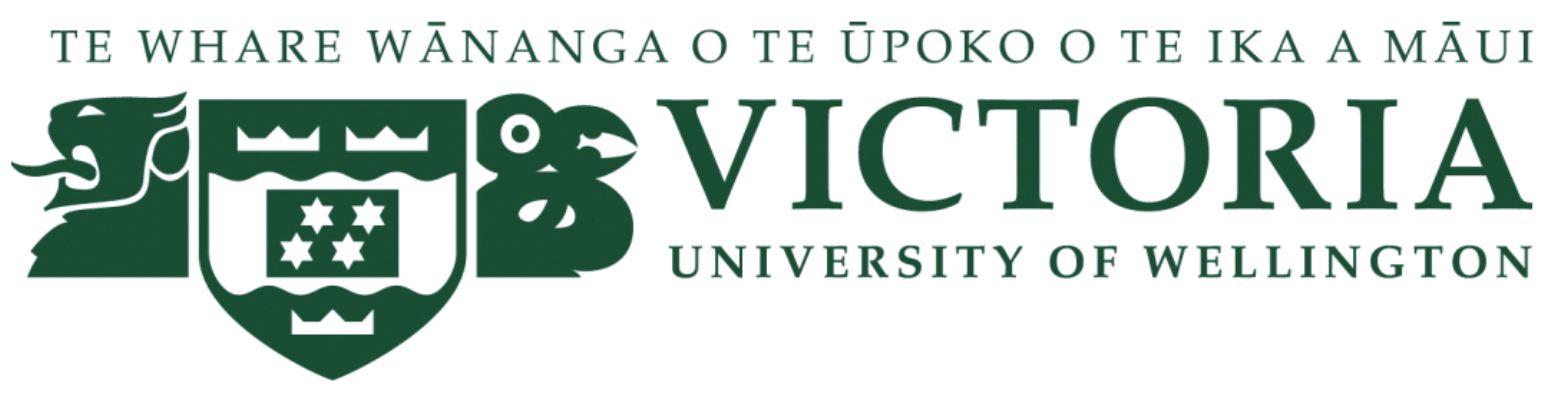

Debriefing Information

\section{Thank you for participating in this study}

Everyone has the occasional unwanted, and unwelcome, thought. It may involve behaviour that one may consider disturbing and very much out of character (e.g., imagining pushing someone in front of a bus), or a negative thought about possible future events (e.g., dying in a house fire). Most people are able to take these thoughts, dismiss them as strange, not act upon them, move on, and not think about them again. For others, however, these thoughts keep coming back again and again. For some people, these recurring unwanted thoughts can be very bothersome, for others, not so much.

The study that you just completed, examines early childhood experiences and the relationship between these experiences and adult mental health. Specifically, we are interested in how early childhood experiences relate to an inflated sense of responsibility (e.g., feeling that things are your responsibility and yours alone) and how this might be related to how well people are able to control unwanted thoughts in their daily lives.

By examining these questions, we can get a better understanding of how these may be related to the development of certain mental health issues which could provide invaluable information in how these might be treated for those who do have such a diagnosis.

If you have any concerns about your own well-being as a result of some of your responses to items within this survey, there are a number of sources from which you could seek further advice, for example meeting with your GP or contacting a peer support worker from Anxiety Support Canterbury (contact details below) to discuss your concerns.

\section{Anxiety Support Canterbury}

www.anxietysupport.org.nz

Phone: (03) 3779665

Email: info@anxietysupport.org.nz

If you have further questions, please feel free to contact either

Kirsty Fraser (Kirsty.Fraser@vuw.ac.nz) or

Associate Professor John McDowall (John.McDowall@ vuw.ac.nz)

at the School of Psychology, Victoria University of Wellington. 
Appendix D

Study 4 Information and Debriefing Information

TE WHARE WĀNANGA O TE ŪPOKO O TE IKA A MĀUI

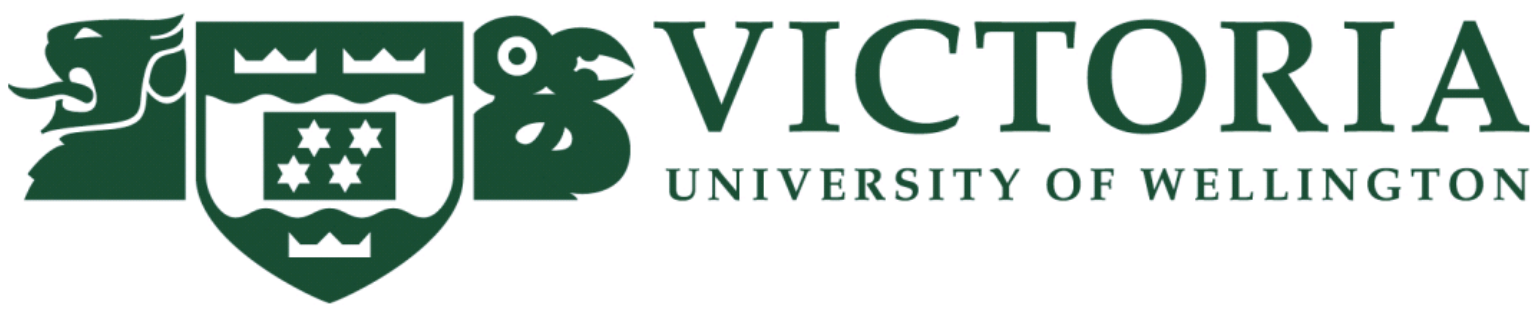

\section{Information and Consent}

This is an online survey investigating aspects about everyday life and mood. This research is being conducted by a $\mathrm{PhD}$ student at Victoria University of Wellington, New Zealand, and it has been approved by the Human Ethics Committee. This survey takes approximately 15-20 minutes to fill out. Please read through the consent form on the following page and click on "I agree" to proceed. This research has been approved by the School of Psychology Human Ethics Committee under delegated authority of Victoria University of Wellington's Human Ethics Committee.

You must be at least 16 years of age to complete the survey.

Thank you for your interest.

Kirsty Fraser \& Associate Professor John McDowall

Victoria University

Wellington

New Zealand

Please read this consent agreement carefully before you decide to participate in the study.

Purpose of the research study: This research aims to develop an understanding of the influences of responsibility and religious beliefs on mental health.

Who is conducting the research?: Kirsty Fraser is a PhD student in the School of Psychology, and this research is supervised by Associate Professor John McDowall and Dr. Matt Crawford This research has been approved by the School of Psychology Human Ethics Committee under delegated authority of Victoria University of Wellington's Human Ethics Committee.

What is involved if you agree to participate?: If you agree to participate in this study, you will be asked to complete the online questionnaire. Some of the questions you 
will be asked may seem very personal. Examples of such questions include those about your childhood experiences and your own thoughts and behaviours. Some items will ask for your perception of experiences such as: "I frequently get nasty thoughts and have difficulty in getting rid of them." Remember, you can withdraw from the study at any time.

You will also be asked for some basic demographic information. We anticipate that your total involvement will take approximately 15-20 minutes.

Protecting your identity: The questionnaire is totally anonymous - your name will never be asked for in this survey. No individual's responses can or would be identified. We will keep the data collected here for at least five years after publication. You will never be identified in this research project or in any other presentation or publication. The information you provide will be coded by number only. In accordance with the requirements of some scientific journals and organisations, your coded data may be shared with other competent researchers. Your coded data may be used in other, related studies. A copy of the coded data will remain in the custody of Associate Professor John McDowall and will be kept in a locked cabinet in his office.

Voluntary participation: Your participation in the study is completely voluntary.

What happens to the information that you provide?: The data you provide may be used for one or more of the following purposes: The overall findings may be submitted for publication in a scientific journal, or presented at scientific conferences; The overall findings may form part of a $\mathrm{PhD}$ thesis, Masters thesis, or Honours research project that will be submitted for assessment.

Who to contact: Regardless of whether or not you complete the survey, if you have any general questions or comments about the research, feel free to contact us. Please note we do not have access to your specific data.

$\underline{\text { Kirsty Fraser }}$

School of Psychology

Victoria University of Wellington

Wellington, New Zealand

E-mail: kirsty.fraser@vuw.ac.nz

Associate Professor John McDowall

School of Psychology

Victoria University of Wellington

Wellington, New Zealand

E-mail: john.mcdowall@vuw.ac.nz

PLEASE FEEL FREE TO PRINT A COPY OF THIS PAGE FOR YOUR RECORDS.

Agreement. Do you agree to participate in the research study described above? YOU MUST BE OVER 16 YEARS OF AGE TO CONTINUE

TO THE SURVEY.

$\ulcorner$ I AGREE

$\ulcorner$ I DO NOT AGREE 


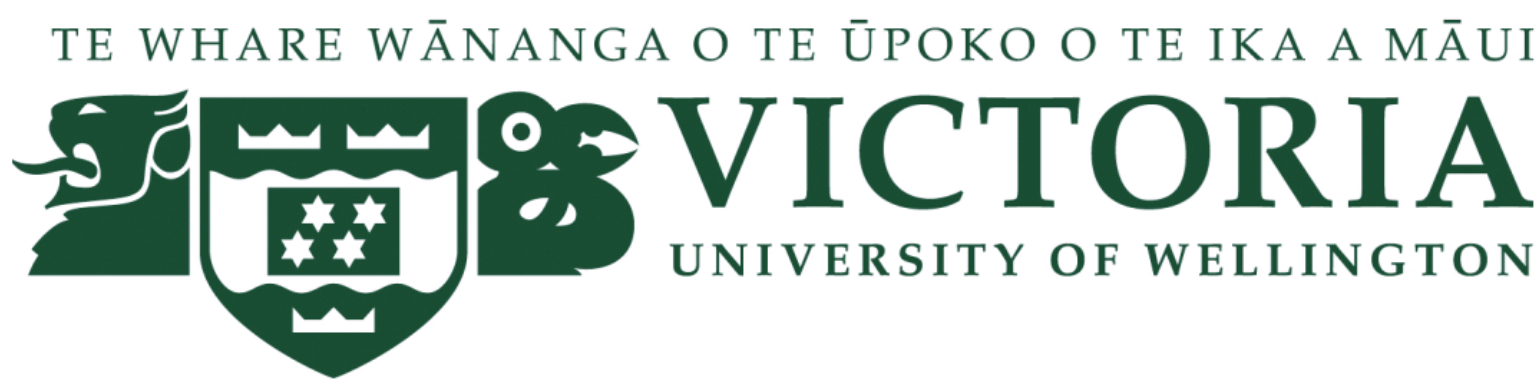

\section{Debriefing Information}

\section{Thank you for participating in this study}

Everyone has the occasional unwanted, and unwelcome, thought. It may involve behaviour that one may consider disturbing and very much out of character (e.g., imagining pushing someone in front of a bus), or a negative thought about possible future events (e.g., dying in a house fire). Most people are able to take these thoughts, dismiss them as strange, not act upon them, move on, and not think about them again. For others, however, these thoughts keep coming back again and again. For some people, these recurring unwanted thoughts can be very bothersome, for others, not so much.

The study that you just completed, examines thoughts and beliefs about personal responsibility and the relationship between these and adult mental health. Specifically, particular types of responsibility beliefs have been associated with anxiety and depression, and interestingly also religiosity. Recent research suggests that when these beliefs are present in those who believe in God, they are not linked with anxiety. The study you just completed hopes to repeat this finding with depression as well. This has important implications for treatment programmes designed to change responsibility beliefs among those with religious beliefs.

It is important to note that the current study is focused on these relationships within the general population rather than looking at individuals who have been diagnosed with a particular mental health issue. By examining these questions within a broad range of people within the population, we can get a better understanding of how these may be related to the development of certain mental health issues which could provide invaluable information in how these might be treated for those who do have such a diagnosis.

If you have any concerns about your own well-being as a result of some of your responses to items within this survey, there are a number of sources from which you could seek further advice. If you are a student, you might wish to contact the student health organisation in your university. If you are not a student, the first step in receiving additional information should be to set up a meeting with your GP to discuss your concerns. Your GP should be able to guide you to the appropriate sources if he or she perceives that there is a problem. Alternatively, you may approach your church leader or elders for help and guidance.

\section{Again, thank you for your participation}


If you have further questions, please feel free to contact either Kirsty Fraser (Kirsty.Fraser@ vuw.ac.nz) or Associate Professor John McDowall (John.McDowall@vuw.ac.nz) at the School of Psychology, Victoria University of Wellington. 


\section{Appendix E}

The Obsessive Compulsive Inventory - Revised (OCI-R; Foa et al., 2002).

The following statements refer to experiences that many people have in their everyday lives.

Circle the number that best describes HOW MUCH that experience has DISTRESSED or BOTHERED you during the PAST MONTH.

I have saved up so many things that they get in the way.

I check things more often than necessary.

I get upset if objects are not arranged properly.

I feel compelled to count while I am doing things.

I find it difficult to touch an object when I know it has been touched by strangers or certain people.

I find it difficult to control my own thoughts.

I collect things I don't need.

I repeatedly check doors, windows, drawers, etc.

I get upset if others change the way I have arranged things.

I feel I have to repeat certain numbers.

I sometimes have to wash or clean myself simply because I feel contaminated.

I am upset by unpleasant thoughts that come into my mind against my will.

I avoid throwing things away because I am afraid I might need them later.

I repeatedly check gas and water taps and light switches after turning them off.

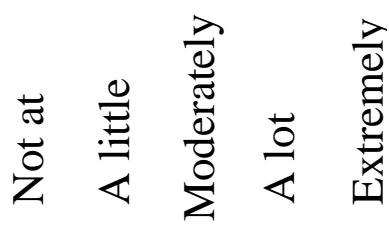

I need things to be arranged in a particular order.

I feel that there are good and bad numbers.

I wash my hands more often and longer than necessary.

$\begin{array}{lllll}0 & 1 & 2 & 3 & 4\end{array}$

$\begin{array}{lllll}0 & 1 & 2 & 3 & 4\end{array}$

$\begin{array}{lllll}0 & 1 & 2 & 3 & 4\end{array}$

$\begin{array}{lllll}0 & 1 & 2 & 3 & 4\end{array}$

$\begin{array}{lllll}0 & 1 & 2 & 3 & 4\end{array}$

I frequently get nasty thoughts and have difficulty in getting rid of them.

$\begin{array}{lllll}0 & 1 & 2 & 3 & 4 \\ 0 & 1 & 2 & 3 & 4 \\ 0 & 1 & 2 & 3 & 4 \\ 0 & 1 & 2 & 3 & 4 \\ 0 & 1 & 2 & 3 & 4 \\ 0 & 1 & 2 & 3 & 4 \\ 0 & 1 & 2 & 3 & 4 \\ 0 & 1 & 2 & 3 & 4 \\ 0 & 1 & 2 & 3 & 4 \\ 0 & 1 & 2 & 3 & 4 \\ 0 & 1 & 2 & 3 & 4 \\ 0 & 1 & 2 & 3 & 4 \\ 0 & 1 & 2 & 3 & 4\end{array}$




\section{Appendix F}

Responsibility Attitude Scale (RAS; Salkovskis et al., 2000).

This questionnaire lists different attitudes or beliefs which people
sometimes hold. Read each statement carefully and decide how much
you agree or disagree with it.

Because people are different, there is no right answer or wrong answer to these statements. To decide whether a given attitude is typical of your way of looking at things, simply keep in mind what you are like MOST of the time.

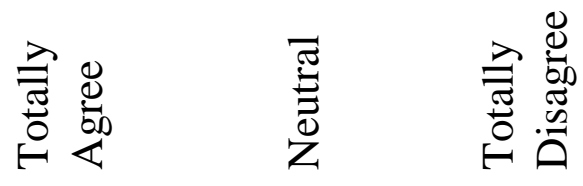

I often feel responsible for things which go wrong

If I don't act when I can foresee danger, then I am to blame for any consequences if it happens

I am too sensitive to feeling responsible for things going wrong

If I think bad things, this is as bad as doing bad things

I worry a great deal about the effects of things which I do or don't do

To me, not acting to prevent disaster is as bad as making disaster happen

If I know that harm is possible, I should always try to prevent it, however unlikely it seems

I must always think through the consequences of even the smallest actions

I often take responsibility for things which other people don't think are my fault

Everything I do can cause serious problems

I am often close to causing harm

I must protect others from harm

I should never cause even the slightest harm to others

I will be condemned for my actions

If I can have even a slight influence on things going wrong, then I must act to prevent it

To me, not acting where disaster is a slight possibility is as bad as making that disaster happen

For me, even slight carelessness is inexcusable when it might affect other people

In all kinds of daily situations, my inactivity can cause as much harm as deliberate bad intentions

Even if harm is a very unlikely possibility, I should always try to prevent it at any cost

Once I think it is possible that I have caused harm, I can't forgive myself

$\begin{array}{lllllll}0 & 1 & 2 & 3 & 4 & 5 & 6\end{array}$

$\begin{array}{lllllll}0 & 1 & 2 & 3 & 4 & 5 & 6\end{array}$

$\begin{array}{lllllll}0 & 1 & 2 & 3 & 4 & 5 & 6\end{array}$

$\begin{array}{lllllll}0 & 1 & 2 & 3 & 4 & 5 & 6\end{array}$

$\begin{array}{lllllll}0 & 1 & 2 & 3 & 4 & 5 & 6\end{array}$

$\begin{array}{lllllll}0 & 1 & 2 & 3 & 4 & 5 & 6\end{array}$

$\begin{array}{lllllll}0 & 1 & 2 & 3 & 4 & 5 & 6\end{array}$

$\begin{array}{lllllll}0 & 1 & 2 & 3 & 4 & 5 & 6\end{array}$

$\begin{array}{lllllll}0 & 1 & 2 & 3 & 4 & 5 & 6\end{array}$

$\begin{array}{lllllll}0 & 1 & 2 & 3 & 4 & 5 & 6\end{array}$

$\begin{array}{lllllll}0 & 1 & 2 & 3 & 4 & 5 & 6\end{array}$

$\begin{array}{lllllll}0 & 1 & 2 & 3 & 4 & 5 & 6\end{array}$

$\begin{array}{lllllll}0 & 1 & 2 & 3 & 4 & 5 & 6\end{array}$

$\begin{array}{lllllll}0 & 1 & 2 & 3 & 4 & 5 & 6\end{array}$

$\begin{array}{lllllll}0 & 1 & 2 & 3 & 4 & 5 & 6\end{array}$

$\begin{array}{lllllll}0 & 1 & 2 & 3 & 4 & 5 & 6\end{array}$

$\begin{array}{lllllll}0 & 1 & 2 & 3 & 4 & 5 & 6\end{array}$

$\begin{array}{lllllll}0 & 1 & 2 & 3 & 4 & 5 & 6\end{array}$

$\begin{array}{lllllll}0 & 1 & 2 & 3 & 4 & 5 & 6\end{array}$

Many of my past actions have been intended to prevent harm to others

I have to make sure other people are protected from all of the consequences of things I do 
Other people should not rely on my judgement

If I cannot be certain I am blameless, I feel that I am to blame

If I take sufficient care then I can prevent any harmful accidents

I often think that bad things will happen if I am not careful enough

$\begin{array}{lllllll}0 & 1 & 2 & 3 & 4 & 5 & 6\end{array}$

$\begin{array}{lllllll}0 & 1 & 2 & 3 & 4 & 5 & 6\end{array}$

$\begin{array}{lllllll}0 & 1 & 2 & 3 & 4 & 5 & 6\end{array}$

$\begin{array}{lllllll}0 & 1 & 2 & 3 & 4 & 5 & 6\end{array}$




\section{Appendix G}

The White Bear Suppression Inventory (WBSI; Wegner \& Zanakos, 1994).

\begin{tabular}{|c|c|c|c|c|c|}
\hline \multirow{2}{*}{$\begin{array}{l}\text { Please indicate the extent to which you agree or disagree with the following } \\
\text { statements. } \\
\text { 1. There are things I prefer not to think about }\end{array}$} & \multicolumn{2}{|c|}{$\begin{array}{l}\text { Strongly } \\
\text { Disagree } \\
\Downarrow\end{array}$} & & \multicolumn{2}{|c|}{$\begin{array}{r}\text { Strongly } \\
\text { Agree } \\
\Downarrow\end{array}$} \\
\hline & 1 & 2 & 3 & 4 & 5 \\
\hline 2. Sometimes I wonder why I have the thoughts I do & 1 & 2 & 3 & 4 & 5 \\
\hline 3. I have thoughts that I cannot stop & 1 & 2 & 3 & 4 & 5 \\
\hline 4. There are images that come to mind that I cannot erase & 1 & 2 & 3 & 4 & 5 \\
\hline 5. My thoughts frequently return to one idea & 1 & 2 & 3 & 4 & 5 \\
\hline 6. I wish I could stop thinking of certain things & 1 & 2 & 3 & 4 & 5 \\
\hline 7. Sometimes my mind races so much I wish I could stop it & 1 & 2 & 3 & 4 & 5 \\
\hline 8. I always try to put problems out of mind & 1 & 2 & 3 & 4 & 5 \\
\hline 9. There are thoughts that keep jumping into my head & 1 & 2 & 3 & 4 & 5 \\
\hline 10. Sometimes I stay busy just to keep thoughts from intruding on my mind & 1 & 2 & 3 & 4 & 5 \\
\hline 11. There are things that I try not to think about & 1 & 2 & 3 & 4 & 5 \\
\hline 12. Sometimes I really wish I could stop thinking & 1 & 2 & 3 & 4 & 5 \\
\hline 13. I often do things to distract myself from my thoughts & 1 & 2 & 3 & 4 & 5 \\
\hline 14. I often have thoughts that I try to avoid & 1 & 2 & 3 & 4 & 5 \\
\hline 15. There are many thoughts that I have that I don't tell anyone & 1 & 2 & 3 & 4 & 5 \\
\hline
\end{tabular}




\section{Appendix $\mathrm{H}$}

Thought-Action Fusion Scale (TAFS; Shafran, Thordarson \& Rachman, 1996).

\begin{tabular}{|c|c|c|c|c|c|}
\hline \multirow{2}{*}{$\begin{array}{l}\text { Do you disagree or agree with the following statements? } \\
\text { 1. Thinking of making an extremely critical remark to a friend is almost } \\
\text { as unacceptable to me as actually saying it }\end{array}$} & \multicolumn{2}{|c|}{$\begin{array}{l}\text { Strongly } \\
\text { Disagree } \\
\Downarrow\end{array}$} & & \multicolumn{2}{|c|}{$\begin{array}{r}\text { Strongly } \\
\text { Agree } \\
\Downarrow\end{array}$} \\
\hline & 1 & 2 & 3 & 4 & 5 \\
\hline $\begin{array}{l}\text { 2. If I think of a relative/friend losing their job, this increases the risk } \\
\text { that they will lose their job }\end{array}$ & 1 & 2 & 3 & 4 & 5 \\
\hline $\begin{array}{l}\text { 3. Having a blasphemous thought is almost as sinful to me as a } \\
\text { blasphemous action }\end{array}$ & 1 & 2 & 3 & 4 & 5 \\
\hline $\begin{array}{l}\text { 4. Thinking about swearing at someone else is almost as unacceptable } \\
\text { to me as actually swearing }\end{array}$ & 1 & 2 & 3 & 4 & 5 \\
\hline $\begin{array}{l}\text { 5. If I think of a relative/friend being in a car accident, this increases the } \\
\text { risk that he/she will have a car accident }\end{array}$ & 1 & 2 & 3 & 4 & 5 \\
\hline $\begin{array}{l}\text { 6. When I have a nasty thought about someone else, it is almost as } \\
\text { bad as carrying out a nasty action }\end{array}$ & 1 & 2 & 3 & 4 & 5 \\
\hline $\begin{array}{l}\text { 7. If I think of a friend/relative being injured in a fall, this increases the } \\
\text { risk that he/she will have a fall and be injured }\end{array}$ & 1 & 2 & 3 & 4 & 5 \\
\hline $\begin{array}{l}\text { 8. Having violent thoughts is almost as unacceptable to me as violent } \\
\text { acts }\end{array}$ & 1 & 2 & 3 & 4 & 5 \\
\hline $\begin{array}{l}\text { 9. If I think of a relative/friend falling ill this increases the risk that } \\
\text { he/she will fall ill }\end{array}$ & 1 & 2 & 3 & 4 & 5 \\
\hline $\begin{array}{l}\text { 10. When I think about making an obscene remark or gesture in church, } \\
\text { it is almost as sinful as actually doing it }\end{array}$ & 1 & 2 & 3 & 4 & 5 \\
\hline $\begin{array}{l}\text { 11. If I wish harm on someone, it is almost as bad as doing harm } \\
\text { 12. If I think of myself being injured in a fall, this increases the risk that I }\end{array}$ & 1 & 2 & 3 & 4 & 5 \\
\hline $\begin{array}{l}\text { will have a fall and be injured } \\
\text { 13. If I think about making an obscene gesture to someone else, it is }\end{array}$ & 1 & 2 & 3 & 4 & 5 \\
\hline $\begin{array}{l}\text { almost as bad as doing it } \\
\text { 14. If I think of myself being in a car accident, this increases the risk that }\end{array}$ & 1 & 2 & 3 & 4 & 5 \\
\hline $\begin{array}{l}\text { I will have a car accident } \\
\text { 15. When I think unkindly about a friend, it is almost as disloyal as doing }\end{array}$ & 1 & 2 & 3 & 4 & 5 \\
\hline an unkind act & 1 & 2 & 3 & 4 & 5 \\
\hline $\begin{array}{l}\text { 16. If I think of myself falling ill, this increases the risk that I will fall ill } \\
\text { 17. If I have a jealous thought, it is almost the same as making a jealous }\end{array}$ & 1 & 2 & 3 & 4 & 5 \\
\hline $\begin{array}{l}\text { remark } \\
\text { 18. Thinking of cheating in a personal relationship is almost as immoral }\end{array}$ & 1 & 2 & 3 & 4 & 5 \\
\hline $\begin{array}{l}\text { to me as actually cheating } \\
\text { 19. Having obscene thoughts in a church is unacceptable to me }\end{array}$ & 1 & 2 & 3 & 4 & 5 \\
\hline
\end{tabular}


Appendix I

Self-Rating Depression Scale (SRDS; Zung, 1965).

Please read each statement and decide how much of the time the statement describes how you have been feeling during the past several days.

1. I feel down-hearted and blue

2. Morning is when I feel the best

3. I have crying spells or feel like it

4. I have trouble sleeping at night

5. I eat as much as I used to

6. I still enjoy sex

7. I notice that I am losing weight

8. I have trouble with constipation

9. My heart beats faster than usual

10. I get tired for no reason

11. My mind is as clear as it used to be

12. I find it easy to do the things I used to

13. I am restless and can't keep still

14. I feel hopeful about the future

15. I am more irritable than usual

16. I find it easy to make decisions

17. I feel that I am useful and needed

18. My life is pretty full

19. I feel that others would be better off if I were dead

20. I still enjoy the things I used to do

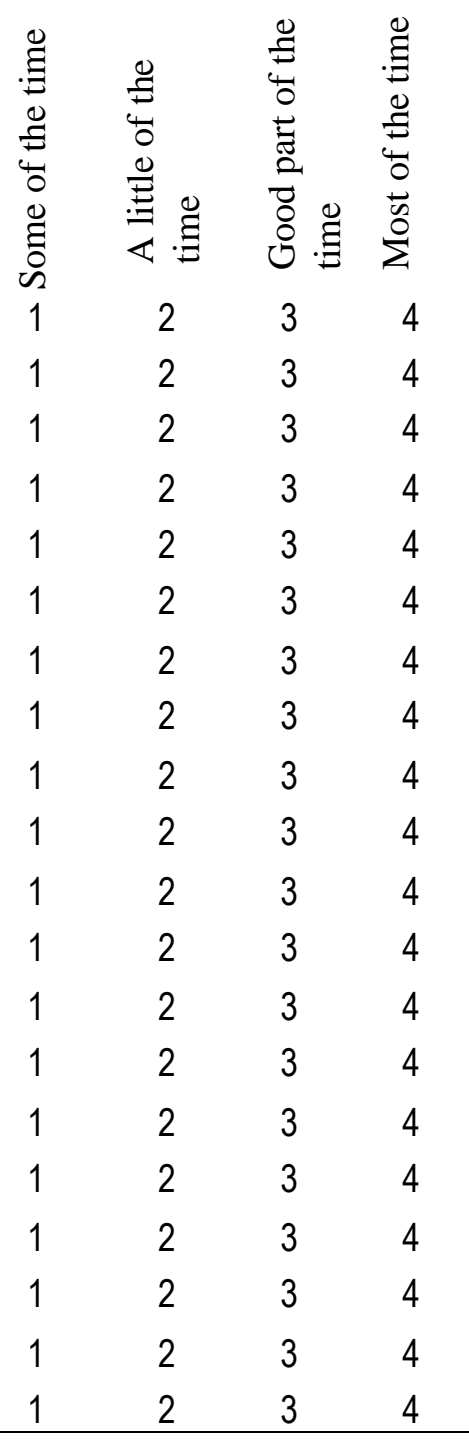


Appendix $\mathbf{J}$

Self-Rating Anxiety Scale (SRAS; Zung, 1971).

Please read each statement and decide how much of the time the statement describes how you have been feeling during the past several days.

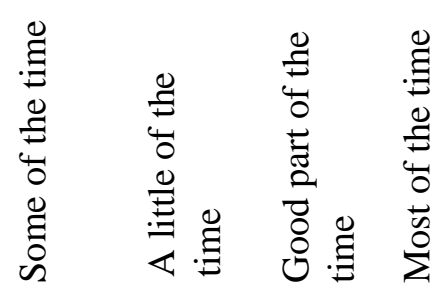

1. I feel more nervous and anxious than usual

2. I feel afraid for no reason

3. I get upset easily or feel panicky

4. I feel like l'm falling apart and going to pieces

5. I feel that everything is all right and nothing bad will happen

6. My arms and legs shake and tremble

7. I am bothered by headaches, neck and back pains

8. I feel weak and get tired easily

9. I feel calm and can sit still easily

10. I can feel my heart beating fast

11. I am bothered by dizzy spells

12. I have fainting spells or feel like it

13. I can breathe in and out easily

14. I get feelings of numbness and tingling in my fingers, toes

15. I am bothered by stomach aches or indigestion

16. I have to empty my bladder often

17. My hands are usually warm and dry

18. My face gets hot and blushes

19. I fall asleep easily and get a good night's rest

20. I have nightmares

$\begin{array}{llll}1 & 2 & 3 & 4 \\ 1 & 2 & 3 & 4 \\ 1 & 2 & 3 & 4 \\ 1 & 2 & 3 & 4 \\ 1 & 2 & 3 & 4 \\ 1 & 2 & 3 & 4 \\ 1 & 2 & 3 & 4 \\ 1 & 2 & 3 & 4 \\ 1 & 2 & 3 & 4 \\ 1 & 2 & 3 & 4 \\ 1 & 2 & 3 & 4 \\ 1 & 2 & 3 & 4 \\ 1 & 2 & 3 & 4 \\ 1 & 2 & 3 & 4 \\ 1 & 2 & 3 & 4 \\ 1 & 2 & 3 & 4 \\ 1 & 2 & 3 & 4 \\ 1 & 2 & 3 & 4 \\ 1 & 2 & 3 & 4 \\ 1 & 2 & 3 & 4\end{array}$




\section{Appendix K}

Pathways to Inflated Responsibility Scale (PIRBS; Coles \& Schofield, 2008).

\begin{tabular}{|c|c|c|c|c|c|}
\hline $\begin{array}{l}\text { This questionnaire asks about what things were like for you as a child. } \\
\text { There are no right or wrong answers; we are just interested in what things } \\
\text { were like for you growing up. Please read each statement carefully and } \\
\text { then circle a response to indicate how frequently that statement was true } \\
\text { for you. }\end{array}$ & $\begin{array}{l}\overline{0} \\
\overrightarrow{0} \\
\ddot{z}\end{array}$ & 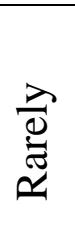 & 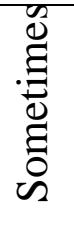 & 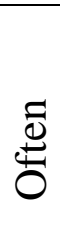 & $\begin{array}{l}\text { 少 } \\
\frac{\pi}{3} \\
\frac{1}{4}\end{array}$ \\
\hline As a child... & $\Downarrow$ & & & & $\Downarrow$ \\
\hline ... I was taught to follow a precise set of rules & 0 & 1 & 2 & 3 & 4 \\
\hline ... I was responsible for protecting a family member/family members & 0 & 1 & 2 & 3 & 4 \\
\hline ... I was taught that rules were to be obeyed without discussion & 0 & 1 & 2 & 3 & 4 \\
\hline$\ldots$ I was responsible for the cooking & 0 & 1 & 2 & 3 & 4 \\
\hline ... my family cares a lot about following rules & 0 & 1 & 2 & 3 & 4 \\
\hline ... I was responsible for keeping our house functioning smoothly & 0 & 1 & 2 & 3 & 4 \\
\hline $\begin{array}{l}\text {... my parent(s) frequently preferred to do things for me rather than have me do } \\
\text { them myself }\end{array}$ & 0 & 1 & 2 & 3 & 4 \\
\hline ... my parent(s) thought that I was unable to deal with danger & 0 & 1 & 2 & 3 & 4 \\
\hline ... my parent(s) strongly valued obedience & 0 & 1 & 2 & 3 & 4 \\
\hline ... my parent(s) thought that I couldn't handle things & 0 & 1 & 2 & 3 & 4 \\
\hline$\ldots$ adults around me strictly enforced rules & 0 & 1 & 2 & 3 & 4 \\
\hline ... my parent(s) thought that I couldn't protect myself & 0 & 1 & 2 & 3 & 4 \\
\hline$\ldots$ I was more like a parent than most kids my age & 0 & 1 & 2 & 3 & 4 \\
\hline ... my parent(s) did many things to protect me & 0 & 1 & 2 & 3 & 4 \\
\hline $\begin{array}{l}\text {.. I had more responsibility for taking care of myself than most kids my age } \\
\text { Sometimes things that we do, or choose not to do, result in serious } \\
\text { misfortune. For example, a surgeon's error may cause harm to a patient. } \\
\text { Or, a mechanics failure to test a car's brakes may lead to an accident. The } \\
\text { misfortune can have a catastrophic effect on the person's health or } \\
\text { welfare. Also, this misfortune can occur to others or us. We are interested } \\
\text { in whether your actions have ever resulted in a serious misfortune } \\
\text { occurring. }\end{array}$ & 0 & 1 & 2 & 3 & 4 \\
\hline $\begin{array}{l}\text { I am confident that something I did resulted in someone else experiencing a } \\
\text { serious misfortune }\end{array}$ & 0 & 1 & 2 & 3 & 4 \\
\hline $\begin{array}{l}\text { I am confident that something / did resulted in me experiencing a serious } \\
\text { misfortune }\end{array}$ & 0 & 1 & 2 & 3 & 4 \\
\hline $\begin{array}{l}\text { I am confident that something I did not do resulted in someone else } \\
\text { experiencing a serious misfortune }\end{array}$ & 0 & 1 & 2 & 3 & 4 \\
\hline $\begin{array}{l}\text { I am confident that something I did not do resulted in me experiencing a serious } \\
\text { misfortune }\end{array}$ & 0 & 1 & 2 & 3 & 4 \\
\hline \multicolumn{6}{|l|}{$\begin{array}{l}\text { Sometimes it appears that something we think or do may have resulted in } \\
\text { a serious misfortune. For example, a child may wish an adult dead and } \\
\text { soon thereafter the adult dies. Therefore, it appears like their thoughts } \\
\text { contributed to the misfortune. We are interested in whether it has ever } \\
\text { appeared that your thoughts or actions have resulted in a serious } \\
\text { misfortune occurring. }\end{array}$} \\
\hline $\begin{array}{l}\text { I believe that something I did or did not do may have contributed to someone } \\
\text { else experiencing a serious misfortune }\end{array}$ & 0 & 1 & 2 & 3 & 4 \\
\hline
\end{tabular}


I believe that something I did or did not do may have contributed to me

0

$\begin{array}{llll}1 & 2 & 3 & 4\end{array}$
experiencing a serious misfortune

I believe that my thoughts may have contributed to someone else experiencing 0

1

2 a serious misfortune

I believe that my thoughts may have contributed to me experiencing a serious 0 1 2 3 4 misfortune 


\section{Appendix L}

Levenson Multidimensional Locus of Control Inventory (Levenson, 1973).

Following is a series of attitude statement. Each represents a commonly held opinion. There are no right or wrong answers. You will probably agree with some items and disagree with others. We are interested in the extent to which you agree or disagree with such matters of opinion. Read each statement carefully. Then indicate the extent to which you agree or disagree.

Whether or not I get to be a leader depends mostly on my ability To a great extent my life is controlled by accidental happenings I feel like what happens in my life is mostly determined by powerful people

Whether or not I get into a car accident depends mostly on how good a driver I am

When I make plans, I am almost certain to make them work

Often there is no chance of protecting my personal interests from bad luck happenings

When I get what I want, it is usually because l'm lucky

Although I might have good ability, I will not be given leadership responsibility without appealing to those positions of power

How many friends I have depends on how a person I am

I have often found that what is going to happen will happen

My life is chiefly controlled by powerful others

Whether or not I get into a car accident is mostly a matter of luck

People like myself have very little chance of protecting our personal

interests when they conflict with those of strong pressure groups

It's not always wise for me to plan too far ahead because many things turn out to be a matter of good or bad fortune

Getting what I want requires pleasing those people above me

Whether or not I get to be a leader depends on whether l'm lucky enough to be in the right place at the right time

If important people were to decide they didn't like me, I probably wouldn't make many friends

I can pretty much determine what will happen in my life

I am usually able to protect my personal interests

Whether or not I get into a car accident depends mostly on the other driver

When I get what I want, it's usually because I worked hard for it

In order to have my plans work, I make sure that they fit in with the desires of people

My life is determined by my own actions

It's chiefly a matter of fate whether or not I have a few friends or many friends

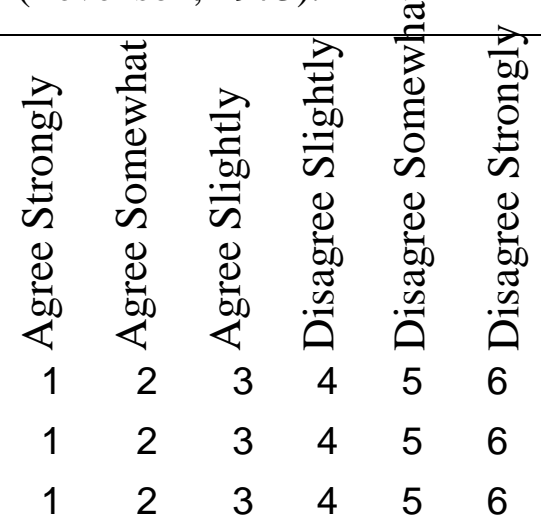

$\begin{array}{llllll}1 & 2 & 3 & 4 & 5 & 6\end{array}$

$\begin{array}{llllll}1 & 2 & 3 & 4 & 5 & 6\end{array}$

$\begin{array}{llllll}1 & 2 & 3 & 4 & 5 & 6\end{array}$

$\begin{array}{llllll}1 & 2 & 3 & 4 & 5 & 6\end{array}$

$\begin{array}{llllll}1 & 2 & 3 & 4 & 5 & 6\end{array}$

$\begin{array}{llllll}1 & 2 & 3 & 4 & 5 & 6\end{array}$

$\begin{array}{llllll}1 & 2 & 3 & 4 & 5 & 6\end{array}$

$\begin{array}{llllll}1 & 2 & 3 & 4 & 5 & 6\end{array}$

$\begin{array}{llllll}1 & 2 & 3 & 4 & 5 & 6\end{array}$

$\begin{array}{llllll}1 & 2 & 3 & 4 & 5 & 6\end{array}$

$\begin{array}{llllll}1 & 2 & 3 & 4 & 5 & 6\end{array}$

$\begin{array}{llllll}1 & 2 & 3 & 4 & 5 & 6\end{array}$

$\begin{array}{llllll}1 & 2 & 3 & 4 & 5 & 6\end{array}$

$\begin{array}{llllll}1 & 2 & 3 & 4 & 5 & 6\end{array}$

$\begin{array}{llllll}1 & 2 & 3 & 4 & 5 & 6\end{array}$

$\begin{array}{llllll}1 & 2 & 3 & 4 & 5 & 6\end{array}$

$\begin{array}{llllll}1 & 2 & 3 & 4 & 5 & 6\end{array}$

$\begin{array}{llllll}1 & 2 & 3 & 4 & 5 & 6\end{array}$

$\begin{array}{llllll}1 & 2 & 3 & 4 & 5 & 6\end{array}$

$\begin{array}{llllll}1 & 2 & 3 & 4 & 5 & 6\end{array}$

$\begin{array}{llllll}1 & 2 & 3 & 4 & 5 & 6\end{array}$ 